\title{
Maintenance of primary human colorectal cancer microenvironment using a perfusion bioreactor-based 3D culture system
}

\author{
Inauguraldissertation \\ zur \\ Erlangung der Würde eines Doktors der Philosophie \\ vorgelegt der \\ Philosophisch-Naturwissenschaftlichen Fakultät \\ der Universität Basel \\ von
}

\section{Celeste Manfredonia}

von Italien

Basel, 2019

Originaldokument gespeichert auf dem Dokumentenserver der Universität Basel edoc.unibas.ch 
Genehmigt von der Philosophisch-Naturwissenschaftlichen Fakultät auf Antrag von

Prof. Dr. Ivan Martin

Prof. Dr. Gerhard M. Christofori

Basel, 26.06.2018

Prof. Dr. Martin Spiess

The Dean of Faculty 
To my father and to Naples, my home town

A mio padre e a Napoli, la mia città 
During my PhD training I have extensively investigated the suitability of a perfusion-based bioreactor culture system to maintain primary colorectal cancer tissues.

This thesis consists of a first chapter that includes a general Introduction covering important aspects related to the development, staging, prognosis and therapies in colorectal cancer and function of the principal components of the tumor microenvironment. Finally, I extensively discuss novel bidimensional and tridimensional in vitro models to predict drug responsiveness in colorectal cancer.

The following three chapters include a comprehensive description of the Methods used and the Results obtained. Major findings are then commented in the Discussion. This part represent a manuscript, which is currently under revision.

Finally, in the Perspectives, I discuss future possible application of the perfused-based bioreactor system. In particular, I report preliminary results obtained with glycogen and human serum albumin nanoparticles, developed using an innovative ultrasonic technique during my visit at the Nanostructured Interfaces and Materials Science Group lead by Professor Frank Caruso at the University of Melbourne, Australia.

During my PhD studies, I also contributed to writing a review focusing on In Vitro Modeling of Tumor-Immune System Interaction that is included as appendix. 


\section{TABLE OF CONTENTS}


SUMMARY. 8

CHAPTER I 12

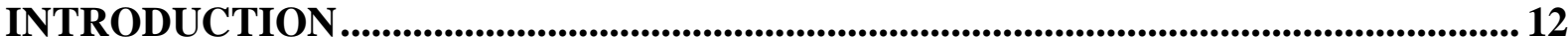

1. Human colorectal cancer .......................................................................................................................... 13

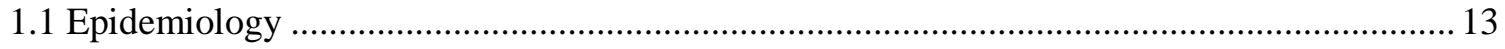

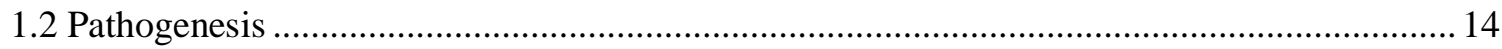

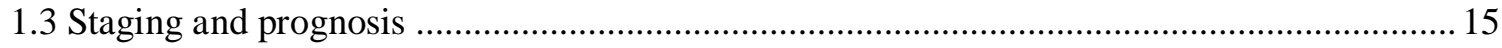

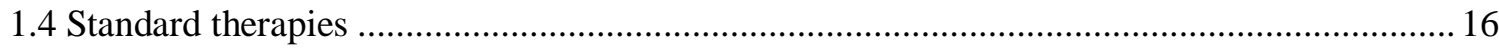

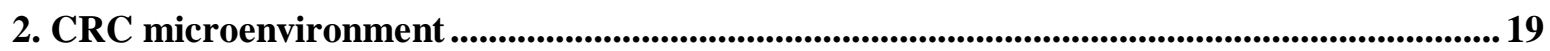

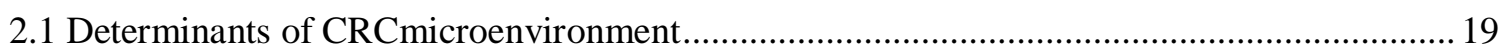

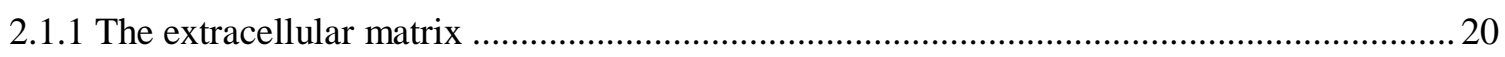

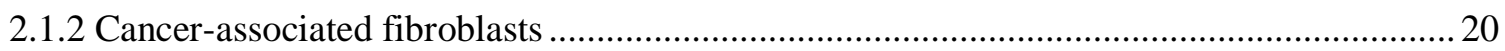

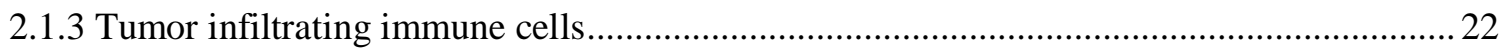

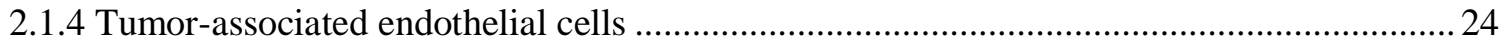

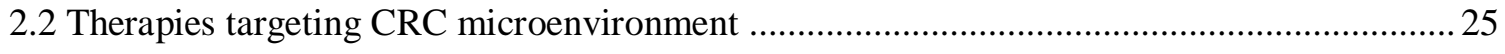

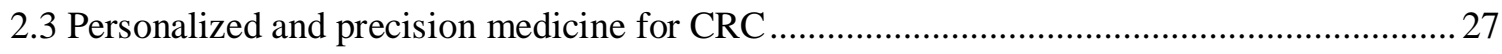

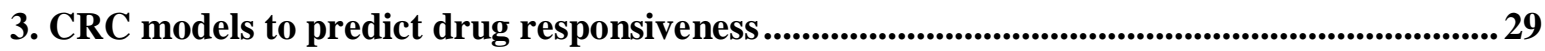

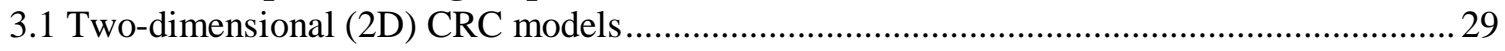

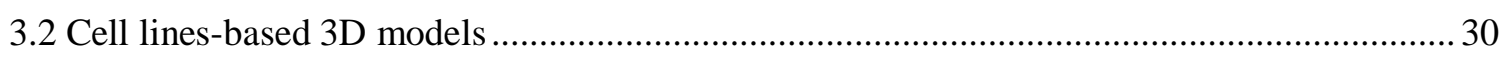

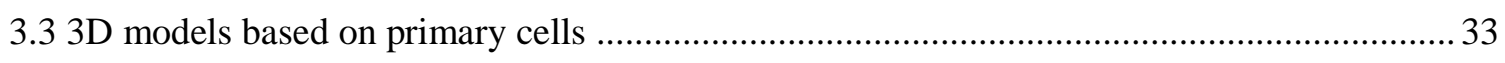

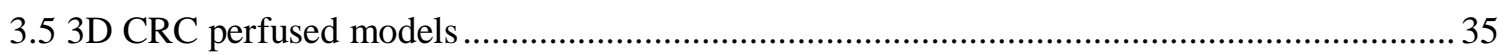

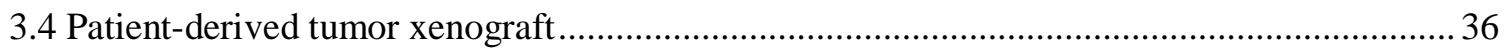

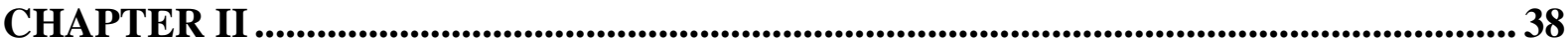

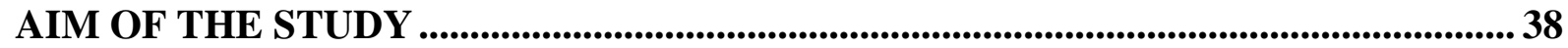

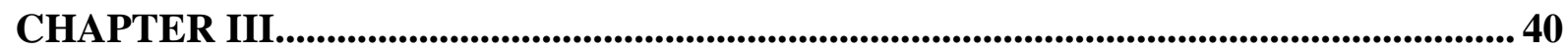

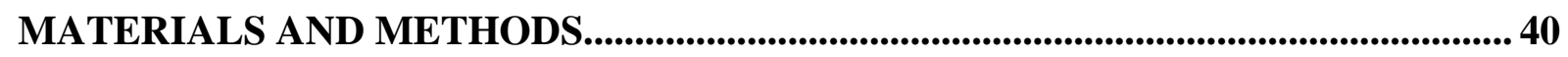

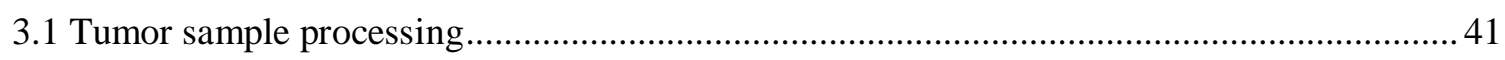

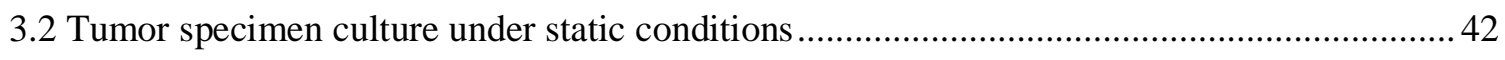

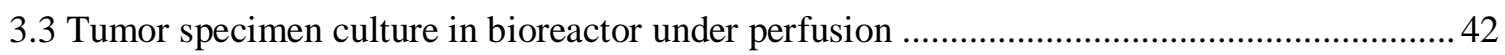

3.4 Histomorphological assessment and immunofluorescence ..................................................... 44

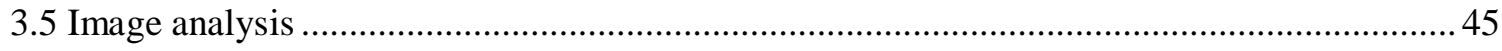

3.6 Assessment of functionality of immune and mesenchymal stromal cells ............................... 47 
RESULTS

4.1 Culture under perfusion preserves the heterogeneity of CRC microenvironment

4.2 Perfused cultures better preserved viability and functionality of all CRC cellular components

4.3 Bioreactor-based cultures are amenable to test drug responsiveness of primary CRC tissues 60

CHAPTER V

DISCUSSION

CHAPTER VI

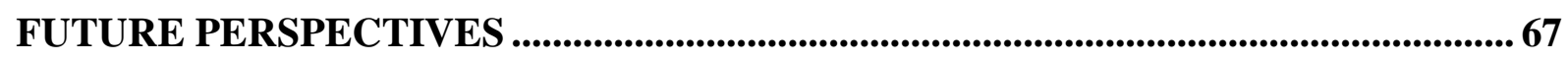

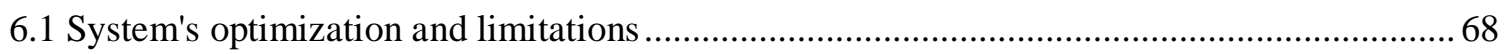

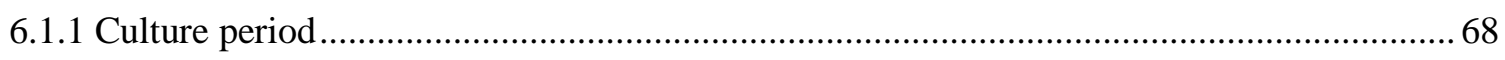

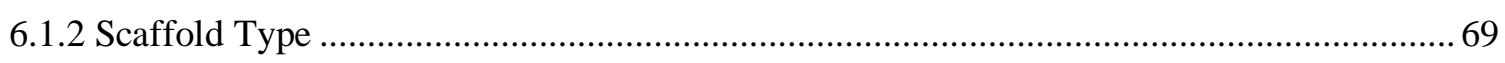

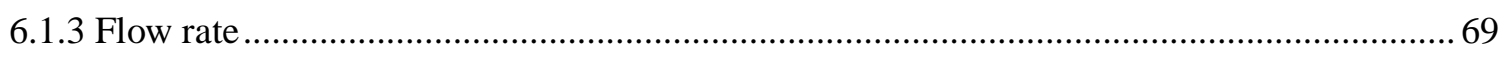

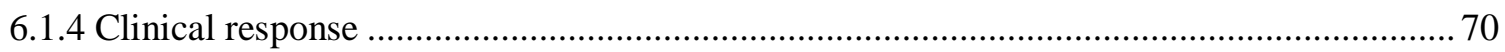

6.2 Suitability of the perfusion-based bioreactor for the assessment of the tumor response to

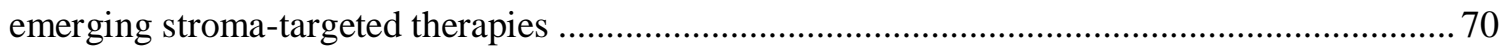

6.2.1 Testing of novel nanoparticles-mediated drug delivery ...................................................... 70

6.2.2 Testing of emerging stroma-targeted therapies ................................................................... 73

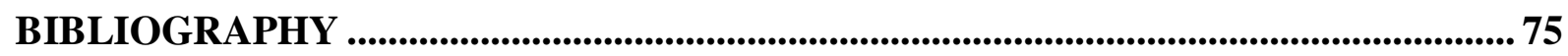

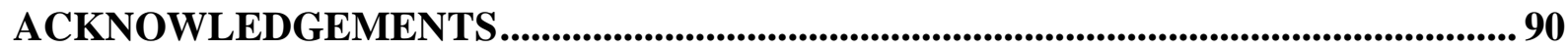

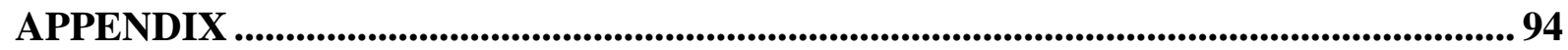


SUMMARY 


\section{$\underline{\text { Introduction }}$}

Colorectal cancer (CRC) is a leading cause of cancer-related death worldwide, often diagnosed in advanced stage. Chemotherapeutic regimens currently in use for human CRC show limited success rates, underlying the need of novel and personalized therapeutic schemes.

A pre-requisite for the development of tailored treatments is the possibility to predict patient responsiveness. However, a major challenge is represented by the lack of adequate in vitro models.

The heterogeneous tissue composition of CRC patients has been recognized to play a key role in response to treatment due to the interaction between cancerous and non-transformed cells within the tumor microenvironment. However, all novel experimental approaches proposed for the evaluation of tumor drug responses, including primary cell cultures or xenotransplantation of cancer specimens in immunodeficient animals, result in loss or dramatic modifications of the tumor microenvironment. Thus, the development of adequate in vitro models allowing maintenance of whole CRC microenvironment is urgently needed.

\section{$\underline{\text { Aim }}$}

During my PhD training, I have investigated the suitability of a perfusion- based bioreactorculture system to maintain primary CRC tissues.

In particular, I addressed:

1. The maintenance of the heterogeneity of CRC microenvironment as compared to static cultures.

2. The assessment of drug responsiveness of primary CRC tissues.

\section{$\underline{\text { Method }}$}

Freshly excised CRC specimens were cut into fragments, inserted between two collagen type I sponges in a "sandwich-like" format and cultured for three days in a perfused-based bioreactor system or under static conditions. 
Fresh tissues, tissues cultured under perfusion and static conditions were weighted and subjected to histomorphological evaluation. Percentage of epithelial cells was evaluated upon hematoxylin and eosin staining. Number of stromal, hematopoietic cells and total cell nuclei were counted using CellProfiler image analysis software following staining for vimentin, CD45, and DAPI, respectively. Viability of tumor cells was assessed upon Ki67 and cleaved caspase 3 staining. The preservation of functionality of tumor-associated stromal cells in perfused cultures was evaluated by assessing release of IL-6 upon stimulation with IL-17. For assessment of immune cells, IL-2 and IFN- $\gamma$ release upon activation with Phytohaemagglutinin was measured. Finally, drug responsiveness of CRC tissue in perfused cultures, was evaluated by assessing proliferation and apoptosis of tumor cells to the conventional chemotherapeutic 5-Fluorouracil, upon Ki67 and cleaved caspase 3 staining, respectively.

\section{$\underline{\text { Results }}$}

Our results showed that $\mathrm{CRC}$ tissues cultured under perfusion preserve the tissue mass at higher extent as compared to static cultures. Moreover, perfused tissues maintained higher tissue cellularity in comparison to static cultures.

Tumor cells cultured under perfusion displayed an almost intact structure, as compared to the original tumors, and were viable and proliferating. In addition, stromal cells were maintained in proportions similar to those of original tumors and fully viable, as indicated by responsiveness to micro-environmental stimuli, such as IL-17. Furthermore, immune cells were also partially preserved, and were capable of releasing effector cytokines, such as IL-2 and IFN- $\gamma$, upon activation by mitogenic stimulation.

In contrast, in cultures performed under static conditions, fewer viable tumor and stromal cells were preserved, whereas immune cells were completely lost. In fact, in static cultures, percentages of proliferating cells were significantly reduced, whereas those of apoptotic cells were significantly increased. 
Importantly, perfusion-based cultures proved suitable for testing the sensitivity of primary tumor cells to chemotherapies of current use in CRC. Indeed, following three days of treatment with 5-fluouracil (5-FU), an overall significant reduction in percentages of epithelial proliferating cells, and a significant increase in the fraction of apoptotic cells could be observed. Notably, analysis of individual samples revealed heterogeneous responses across different tumors.

\section{Conclusions}

Our results cumulatively suggest that primary CRC culture under perfusion preserve the microenvironment with its native tissue architecture and composition. Importantly, our culture system also preserves viability and functionality of non-transformed cells, including mesenchymal stromal cells and tumor infiltrating lymphocytes.

Moreover, bioreactor-based cultures are amenable for testing sensitivity of primary CRC tissues to currently used chemotherapies and reveal heterogeneous responsiveness across different samples.

Thanks to its capacity to maintain TME heterogeneity, our system may allow personalized drug testing within a more physiological context. Our culture system may also prove suitable for testing therapies whose efficacy is influenced by whole TME, such as drug-loaded nanoparticles and emerging stroma-targeted therapies currently under clinical investigation for CRC. Furthermore, we envisage validating its ability to predict patient-specific clinical responses in the context of follow-up studies. 
CHAPTER I

INTRODUCTION 


\section{Human colorectal cancer}

\subsection{Epidemiology}

Colorectal Cancer (CRC) is the fourth most common cause of cancer-related death and the third major cause of morbidity and mortality worldwide ${ }^{1}$.

From 1998 to 2005 CRC incidence rates have declined which might be attributed to improved screening programs for the detection of precancerous polyps. However, CRC incidence and mortality remains still very high. It is, in fact, estimated that every year over 394,000 deaths from CRC still occur worldwide ${ }^{1}$.

Over 14.1 million cases recorded in 2002 all over the world ${ }^{2}$. However, CRC is not uniformly distributed throughout the world. In particular, CRC mainly occurs in developed countries. Indeed, countries with the highest incidence rates include Australia, New Zealand, Canada, the United States and Europe. Modifiable risk factors associated with CRC incidence include physical inactivity, over-weight, cigarette-smoking, alcohol intake and diets reach in fats and poor in calcium, fibers, fruits and vegetables typical of the Western diet. Nonmodifiable risk factors include age ${ }^{1}$, genetic factors ${ }^{3}$, gender and race/ethnicity ${ }^{4}$. In particular, most of CRC are diagnosed after the age of 40 and in particular occurs in people aged 50 or older ${ }^{1,5,6}$.

It affects more women than men with CRC incidence and mortality has been reported to be slightly higher in women $(10.1 \%)$ than in men $(9.4 \%)^{1,7}$. Moreover, women have a higher risk of developing right-sided (proximal) colon cancer than men, which is associated with more aggressive form of neoplasia compared to left-sided (distal) colon cancer ${ }^{8}$.

Race/ethnic disparities among the colorectal cancer population is well documented with nonHispanic Black males and females having the highest incidence and mortality, and Hispanics/Latino females and American Indian/Alaskan Native males having the lowest rates $^{9}$. 
In addition, a history of previous colon polyps, inflammatory bowel disease ${ }^{10}$, such as ulcerative colitis or Crohn's disease, or hereditary factors are associated with the increase in the incidence of $\mathrm{CRC}^{5}$.

\subsection{Pathogenesis}

$\mathrm{CRC}$ arises as the result of an ordered series of events beginning with the transformation of normal epithelium into an invasive adenocarcinoma.

Most cases (88\% - 94\%) of CRC are sporadic ${ }^{11}$ and develop through different pathways of molecular events characterized by inactivation of tumor suppressor genes and oncogenes according to the adenoma-carcinoma sequence model, proposed by Fearon and Vogelstein in 1990 (Figure I.1).

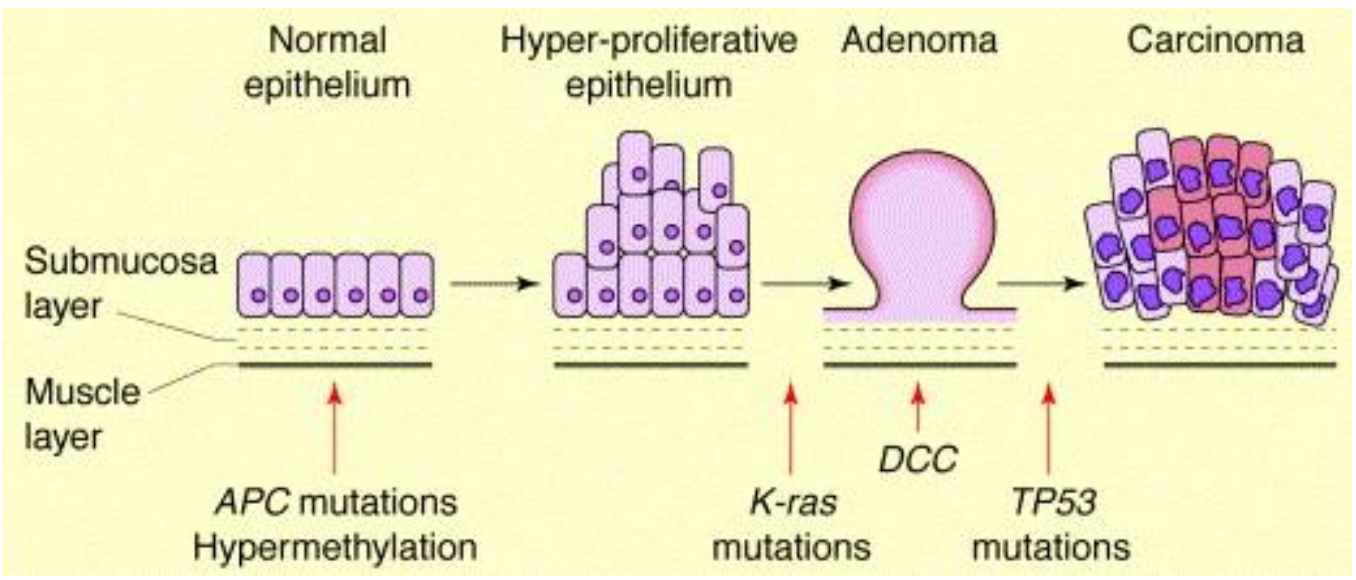

Figure I.1 - Adenoma to carcinoma sequences ${ }^{12}$

Mutations of APC occur in a high proportion of sporadic colorectal carcinomas (up to $80 \%$ ) 13. Inactivation of both copies (alleles) of the APC gene located on chromosome 5 constitutes an early event in colorectal tumorigenesis ${ }^{14}$. The canonical tumor suppressor function of APC is to form a "destruction complex" with Axin/Axin2 and GSK-3 $\beta$ that promotes the ubiquitination and subsequent degradation of the oncogene $\beta$-catenin in the absence of Wnt signaling. Loss of APC function results in an accumulation of $\beta$-catenin, which translocates to 
the nucleus and lead to stimulation of cellular growth and proliferation and to the disruption of differentiation programs ${ }^{15}$.

5-10\% of CRC are hereditary ${ }^{11}$, such as the familial adenomatous polyposis (FAP) and hereditary nonpolyposis colorectal cancer (HPNCC).

The HPNCC, also known as Lynch Syndrome, is the most common form of hereditary cancers and is characterized by the alteration in at least six of the responsible mismatch repair (MMR) genes including MSH2, MSH3, MSH6, MLH1, PMS1, and PMS2 ${ }^{16}$. The mutation of the MMR genes leads to an accumulation of DNA replication errors. This phenomenon is known as microsatellite instability (MSI) and can be identified in more than $90 \%$ of CRC affect by the Lynch syndrome. According to their MS status, CRC MSI can be further classified into MSI-high (MSI-H), MSI-low (MSI-L), or microsatellite-stable.

Epigenetic mechanisms are also involved in the colorectal carcinogenesis and might impact on MS status. For instance, the so called " $\mathrm{CpG}$ island methylator phenotype "(CIMP) refers to a subset of tumors which present a methylation of $\mathrm{CpG}$ islands resulting in inactivation of one of several tumor suppressor genes or other tumor-related genes. Notably, most CIMP CRCs are characterized by promoter $\mathrm{CpG}$ island methylation of the mismatch repair gene, MLH1, resulting in its transcriptional inactivation ${ }^{17}$. These CRCs, although sporadic, are also characterized by MSI-H status ${ }^{18}$.

\subsection{Staging and prognosis}

CRC cancer staging, proposed by the American Joint Committee on Cancer (AJCC) and the International Union for Cancer Control (UICC), is based on histopathologic evaluation and operative findings of excised tissues, i.e., the local invasion depth ( $\mathrm{T}$ stage), lymph node involvement ( $\mathrm{N}$ stage), and presence of distant metastases ( $\mathrm{M}$ stage). TNM classification is used to predict prognosis and provides the basis for therapeutic decisions. CRC survival ranges from $90 \%$ for stage I CRC to $10 \%$ in patients with stage IV disease ${ }^{1}$. However, 
patients with early stage may have approximately $20-30 \%$ risk of recurrence indicating that the currently used TNM staging system may not reflect the individual cancer aggressiveness 19.

Several efforts have been made regarding the identification of tumor related features that might represent further important prognostic factors such as lymphocytic infiltration, venous invasion, circumferential margin status, and tumor budding (i.e., presence of single cells or small clusters at the invasive margin) ${ }^{20}$. In addition, MSI analysis can also provide important information about prognosis and therapy response. Patients with MSI-H show a better prognosis as compared to patients with MSS tumors ${ }^{21}$. Moreover, MSI-H CRCs do not benefit from adjuvant therapy (see below) with 5-fluorouracil (5-FU) whereas show an improved response to irinotecan-based chemotherapy ${ }^{22}$.

\subsection{Standard therapies}

For patients with early stage CRCs (stage I - II), surgery is the only treatment option. Neoadjuvant radiotherapy is reserved for patients with rectal cancer whereas postoperative chemotherapy is usually a postoperative treatment option for patients with positive lymph nodes (stage III or high-risk stage II CRC) and the front-line treatment for stage IV patients.

The most of in use standard therapies target tumor cells o tumor cell-derived factors. In particular, the common standard therapy is represented by a combination of chemotherapy agents, mainly including 5-FU, a fluorinated pyrimidine that inhibit the action of the thymidylate synthase, an enzyme involved in pyrimidine nucleotide synthesis, thus stopping DNA replication.

5-FU is usually combined with leucovorin, a reduced folate, which enhance the inhibition of DNA synthesis by stabilizes the binding of 5-FU to thymidylate synthase, and with oxaliplatin, a platinum derivative that forms inter- and intra- strand cross links in DNA, which prevent DNA replication and transcription, thus inducing cellular apoptosis. The combination 
of 5-FU, leucovorin and oxaliplatin is known as FOLFOX. Irinotecan is also used in CRC treatment and its mechanism of action is based on the inhibition of the topoisomerase I, an enzyme involved in the uncoiling of DNA during replication and transcription. Irinotecan is used in CRC treatment either alone or in combination with leucovorin and 5-FU. This regimen is known as FOLFIRI.

While 5-FU used as single agent in patients with metastatic colorectal cancer has an objective response rate around $20 \%$, the administration of FOLFOX or FOLFIRI ${ }^{23}$ results in significantly increased response rates up to $40 \%$ of treated patients ${ }^{24,25}$. However, the side effects of systemic therapy such as myelotoxicity, neurotoxicity or gastrointestinal toxicity may lead to complications which may affect impact on the quality of life of the patients ${ }^{23}$. Besides these cytotoxic chemotherapies newly targeted strategies have been recently introduced, aiming at interrupting cellular pathways essential for tumor growth and survival. An example is represented by antibodies blocking the epidermal growth factor receptor (EGFR), such as Cetuximab and panitumab ${ }^{26}$, which have been approved for treatment of metastatic colorectal cancer as single agents or in combination with other chemotherapies.

Currently, two epidermal growth factor receptor antagonists: Cetuximab and panitumab 26 have been approved for treatment of metastatic colorectal cancer as single agents or in combination with other chemotherapies. According to the results of the international ASPECCT trial, the first randomized phase III study evaluating the two EGFR-targeted monoclonal antibodies in patients with chemorefractory KRAS wild-type metastatic colorectal cancer (mCRC) metastatic CRC, Panitumumab (Vectibix) proved non-inferior to cetuximab (Erbitux) in extending overall survival (OS) ${ }^{27}$. The response rate when panitumumab is associated with irinotecan is $34 \%$ while it is $20 \%$ when cetuximab is administrated in combination with irinotecan ${ }^{28}$.

However, anti-EGFR antibodies are not effective in the presence of mutations of the rat sarcoma virus (RAS) family gene, including KRAS, NRAS and HRAS, which, under normal 
conditions, regulate the extracellular signaling to deliver growth signals from the epithermal growth factor (EGFR) to the nucleus. In tumor, RAS mutations lead to the disruption of the normal signaling pathway and so to aberrant growth and metastasis. It has been estimated that RAS mutations occur in $30-40 \%$ of CRC ${ }^{29}$.

Another targeted treatment which has been used in combination with standard chemotherapeutic agents in several clinical trials in patients with advanced CRC is the angiogenesis inhibitor Bevacizumab ${ }^{30}$, a humanized antibody directed against the vascular endothelial growth factor (VEGF). Results showed that Bevacizumab resulted in an improved tumor response as compared with fluorouracil and leucovorin alone ${ }^{31}$.

Targeting angiogenesis has also been achieved by using tyrosine kinase inhibitors (TKIs).

However, the role of the TKIs is not yet fully clarified ${ }^{32}$ and a better understanding of novel combinations with TKIs that could prove more efficacious are urgently needed ${ }^{33}$.

In conclusion, the introduction of more complex protocols in addition to 5-FU, as well as the development of targeted therapies has resulted in a significant improvement in the treatment options for CRC. However, to find a system which may reliably predict responsiveness of individual patients to different drugs remains a critical need. 


\section{CRC microenvironment}

\subsection{Determinants of CRCmicroenvironment}

The tumor microenvironment (TME) comprises tumor cells and non-transformed cells, including immune cells, endothelial cells, and stromal fibroblasts, and the extracellular matrix (ECM). These cells communicate through a complex network of interactions such as through a direct cell-cell contact or through soluble and insoluble factors and signaling molecules.

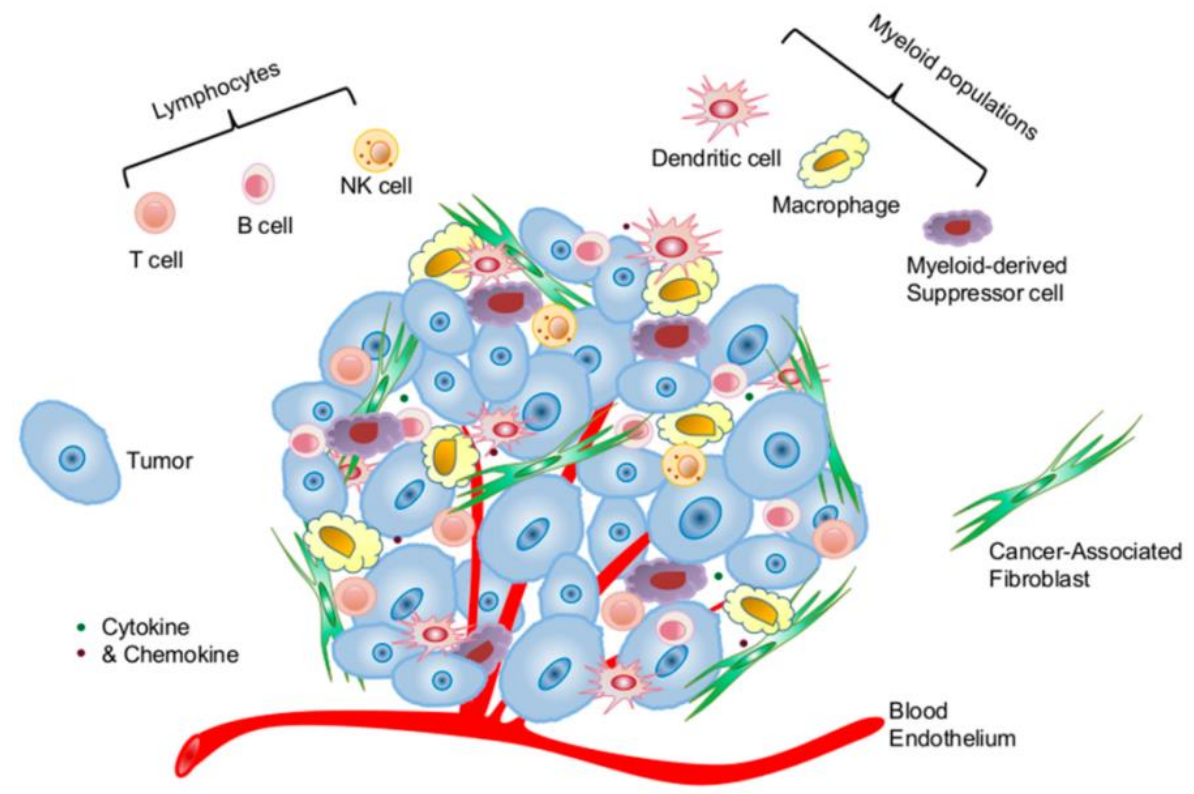

Figure I.2 - Cellular constituents of the tumor microenvironment. The tumor microenvironment consists of complex cellular and molecular and constituents. The cellular constituents consist of immune cells of hematopoietic origin and stromal cells of nonhematopoietic origin. The immune cell compartment comprises tumor-infiltrating lymphocytes and tumor-associated myeloid populations. The stromal compartment consists of cancer-associated fibroblasts and endothelial cells of the lymphatic and blood vasculature ${ }^{34}$

It is now well recognized that non-malignant cells of the TME have a heavily impact on tumor development and progression in all the stages of CRC carcinogenesis ${ }^{35}$. Therefore, to develop novel effective therapies, it is necessary to have a clear understanding of the TME key players in tumor progression. 


\subsubsection{The extracellular matrix}

The extracellular matrix (ECM) is an organized three-dimensional structure that provides a structural scaffold for cells, regulates cell migration, differentiation and proliferation. The ECM is composed by five macromolecules: the most abundant component is collagen followed by laminins, fibronectin proteoglycans, glycosaminoglycans and hyaluronans. Changes in ECM composition lead to changes in physical cues such as rigidity and cells respond to these forces through changes in cell division, migration, gene expression. Tumors are usually stiffer than the surround healthy tissue.

In fact, a recent study demonstrates that the accumulation of P-selectin-mediated platelet upregulates the ECM modifying enzyme lysyl oxidase (LOX) which mediates the cross-linking of collagen and elastin thus increasing the ECM stiffness. Therefore, a stiffer matrix drives CRC development and progression and might be associated with poor prognosis ${ }^{36}$.

\subsubsection{Cancer-associated fibroblasts}

Fibroblasts are responsible for the ECM secretion and they are the main component of the reactive stroma in primary and metastatic CRC where they are known as cancer-associated fibroblasts (CAFs).

CAFs are a heterogeneous population of cells with various origins, the majority of which are derived from resident fibroblasts ${ }^{37}$. CAFs may also be derived from other cells, including mesenchymal stem cells (MSCs), pericytes, adipocytes and endothelial cells, or epithelial cells via epithelial-mesenchymal transition (EMT) ${ }^{38}$. CAFs in the tumor stroma can be differentiated according to their morphology and specific identifiable markers. CAFs are generally presented as large spindle-shaped cells similar to smooth muscle cells (myofilaments and electron dense patches). Commonly used markers to identify CAFs are $\alpha$ SMA, fibroblast activation protein- $\alpha$ (FAP- $\alpha$ ), fibroblast-specific protein-1 (FSP-1/S100A4), or platelet-derived growth factor receptor- $\beta$ (PDGFR- $\beta)^{39}$. Numerous previous studies have 
highlighted a pro-tumorigenic role for CAFs that can stimulate tumor cell proliferation, survival, migration, and invasion via secretion of various growth factors, cytokines, such as hepatocyte growth factor HGF, TGF- $\beta$, interleukin-6 (IL-6), stromal cell-derived factor-1 $\alpha$, IL-1 $\beta$ and TNF $\alpha^{40}$, and chemokines such as CXCL12, CXCL14 and CCL5.

In particular, levels of IL-6 in serum has been associated with poor patient prognosis, possibly due to its ability to promote tumor cell survival and support the production of vascular endothelial growth factor (VEGF).

TGF- $\beta$ has been recognized to have an oncogenic activity by inducing epithelial-tomesenchymal transition (EMT), which is characterized by a change in cell shape from a polarized epithelial cell to a flattened fibroblast-like cell, a decrease in cell-cell contacts and increased cell motility, which in turn enhances tumor cell migration. In addition, TGF- $\beta$ can also indirectly influence tumor growth by inducing fibroblast differentiation, thus leading to tumor tissue remodeling and supporting tumor cell growth. Moreover, TGF- $\beta$ induce the expression of VEGF and connective tissue growth factor (CTGF), thus enhancing the recruitment of endothelial cells to the tumor, leading to the formation of new vessels and allowing tumor growth ${ }^{41}$.

In addition, CAFs secrete ECM and proteases, such as matrix metalloproteases, cathepsins, and plasminogen activators, and thereby induce EMT and promote invasive growth of colon cancer cells ${ }^{38}$. In particular, by expressing elevated amounts of matrix metalloproteinases, CAFs play a critical role in the degradation of the basal membrane (BM). The loss in integrity of the BM allows for invasion by cancer cells which migrate freely through the gaps formed in BM, thus promoting cancer metastasis ${ }^{42}$.

Herrera et al. demonstrated that patients with high "CAF signature" had a remarkably poor prognosis ${ }^{43}$. Moreover, expression of FAP has been shown to be associated with an aggressive disease and to be an independent negative prognostic factor in CRC patients ${ }^{44,45}$. 
Calon et al. found that active TGF- $\beta$ signaling in CAFs increases the frequency of tumorinitiating cells, whereas, in patient-derived tumor organoids and xenografts, inhibitors of TGF- $\beta$ signaling block the cross-talk between cancer cells and fibroblasts and prevent metastatic spread $^{46}$.

Isella et al. confirmed that the CAF signature was associated with poor prognosis in untreated colon cancer patients and also predicted resistance to radiotherapy in rectal cancer ${ }^{47}$.

Taken together these studies confirmed that CAFs significantly contributes to clinical features of CRC and shapes the therapeutic response.

\subsubsection{Tumor infiltrating immune cells}

The immune cell types that may be observed within the TME include macrophages, dendritic cells, mast cells, natural killer (NK) cells, naïve and memory lymphocytes, B cells and T cells (e.g. $\mathrm{T}$ helper $1\left(\mathrm{~T}_{\mathrm{H}} 1\right), \mathrm{T}_{\mathrm{H}} 2, \mathrm{~T}_{\mathrm{H}} 17$ cells, regulatory $\mathrm{T}\left(\mathrm{T}_{\mathrm{reg}}\right)$ cells, $\mathrm{T}$ follicular helper $\left(\mathrm{T}_{\mathrm{FH}}\right)$ and cytotoxic T cells). The number of infiltrating immune cells is extremely variable within the same tumor type, from different tumor locations and from patient to patient.

T lymphocytes and macrophages are present at the core and invasive tumor margins. Whereas NK cells, myeloid-derived suppressor cells (MDSCs), mast cells and neutrophils are mostly located in the invasive margins. Finally, B lymphocytes and mature dendritic cells preferentially populate tertiary lymphoid islets (TLS) ${ }^{48}$.

The nature, location, functional orientation and density of the different tumor infiltrating immune cell populations is defined as "immune contexture". A fairly new and promising concept is that the immune contexture can yield information that is relevant to prognosis and prediction of a treatment response.

Tumor infiltration by adaptive immune cells, in particular effector $\mathrm{T}$ lymphocytes, has been reported to be associated with clinical outcome. 


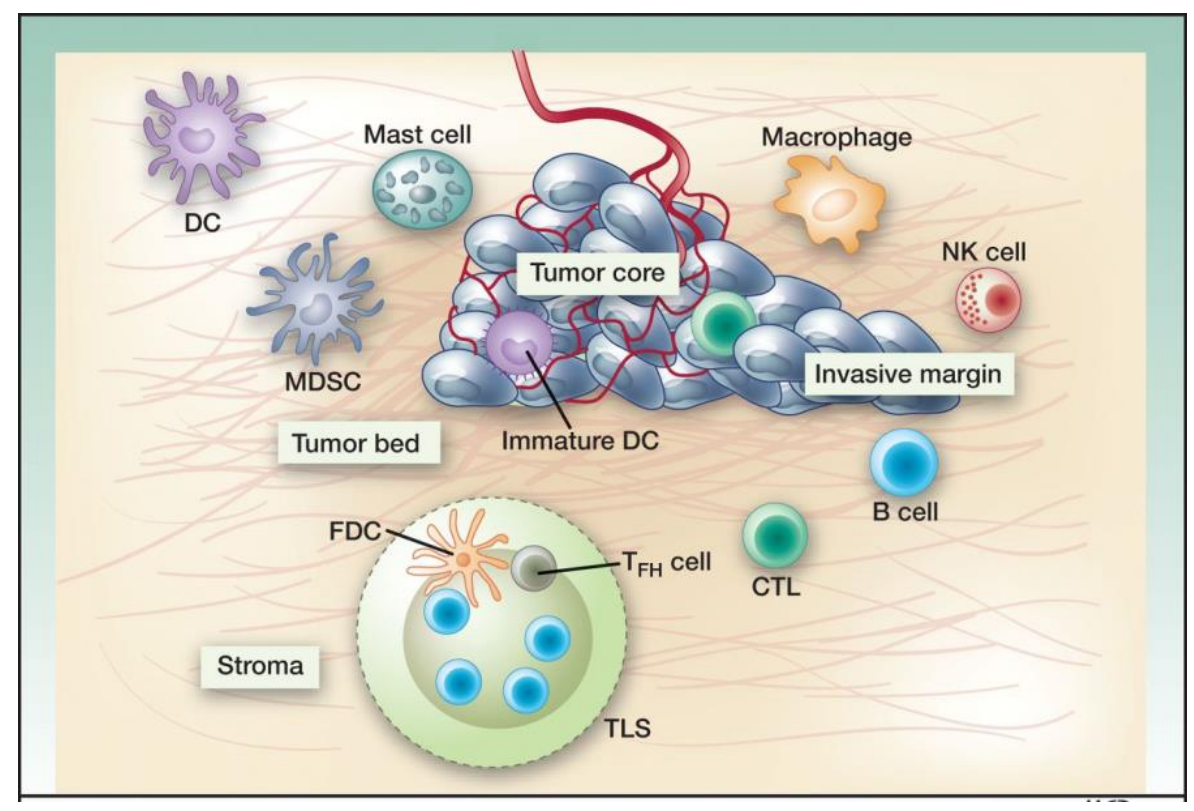

Figure I.3 - The immune contexture The immune cell compartment comprises tumorinfiltrating lymphocytes of $T, B$, and natural killer cells and tumor-associated myeloid populations of dendritic cells, macrophages, and myeloid-derived suppressor cells ${ }^{49}$.

Ropponen et al. showed an inverse correlation between the presence of tumor-infiltrating lymphocytes (TILs) and tumor stage, i.e. TILs are more present in early stages (stage I and II) and decrease in advanced stages (stage III and IV) ${ }^{50}$.

More recently, it has been demonstrated that a high infiltration by CD3+, CD8+ and $\mathrm{CD} 45 \mathrm{RO}+$ cells at the core and invasive margin, clearly correlates with favorable prognosis $51,52$.

These studies have led to the development of the "Immunoscore", a mean of measuring T cell infiltrate into CRCs. The "Immunoscore" has shown to predict outcome more accurately than staging systems currently in use ${ }^{53}$.

Innate immune responses, particularly those involving tumor associated macrophages (TAMs), have been studied and data show that the frequency of these cells infiltrating the tumor can be associated with poor patient outcome, although this is controversial ${ }^{53}$. In contrast, decreased number of preoperative NK cells in patients with CRC was associated 
with an increased frequency of postoperative tumor recurrence ${ }^{54}$. Moreover, recent studies from our group showed that infiltration by neutrophils of the TME enhances the prognostic significance of colorectal cancer infiltration by CD8+ T cells, thus suggesting that they might effectively promote antitumor immunity ${ }^{55}$. In addition, CRC infiltration by myeloperoxidaseexpressing neutrophil granulocytes is associated with favorable prognosis ${ }^{56}$.

\subsubsection{Tumor-associated endothelial cells}

Additional cellular components of the TME include the endothelial cells, which are responsible for the formation of vascular structures which provide nutrients and oxygen. The induction of angiogenesis is an important early event in CRC development thus the microvascular density represents an important prognostic factor and might guide the therapeutic decision. Tumors are characterized by an overexpression of pro-angiogenic factors which lead to a structurally and functionally abnormal vasculature with leaky and tortuous blood vessels. This disorganized vasculature lead to an abnormal TME characterized by large hypoxic zones known to be much more resistant to several cytotoxic drugs than normoxic regions ${ }^{57}$. Also leaking vessels generate elevate interstitial hypertension as well as an impaired blood flow which interfere with the delivery of therapeutics and lead to a heterogeneous microenvironment. In addition, solid tumors present a very poor lymphatic network than normal tissues, which contributes to the increased interstitial fluid pressure (Figure I.4). 

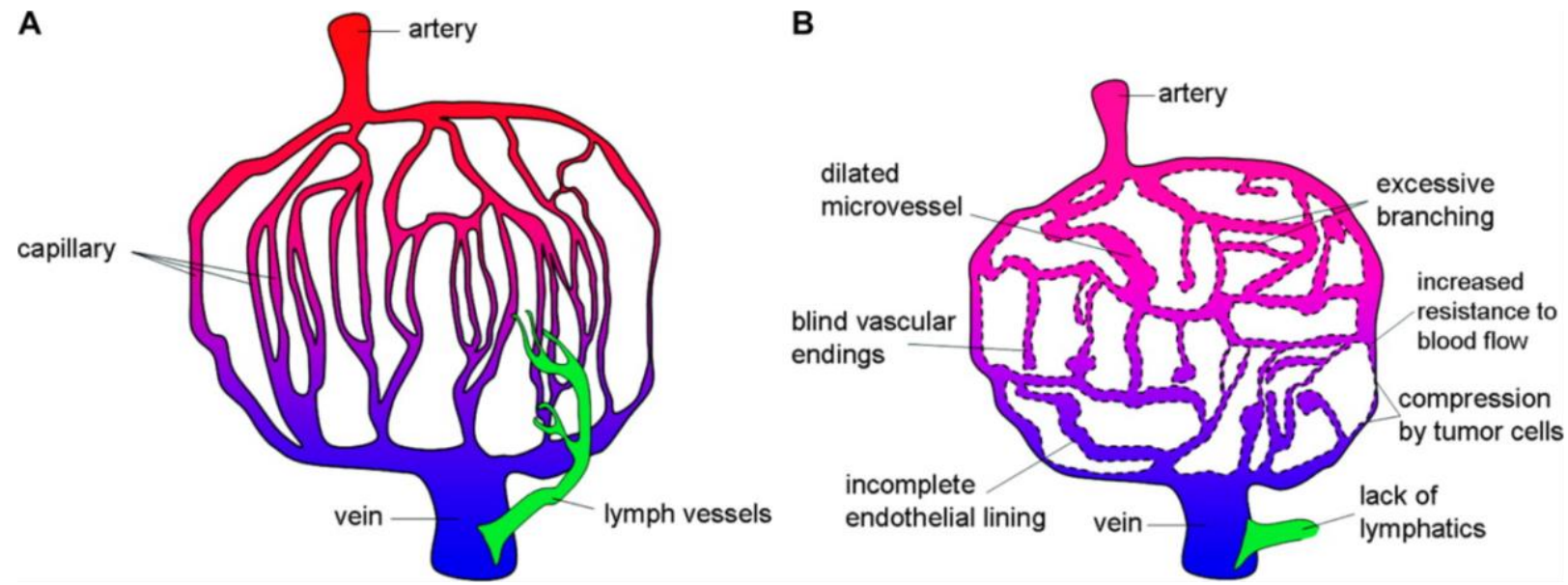

Figure I.4 - Diagrammatic representation of the vascular system. A ) Normal tissue. B)

Solid tumor. Red represents well-oxygenated arterial blood, blue represents poorly oxygenated venous blood, and green represents lymphatic vessels ${ }^{58}$.

\subsection{Therapies targeting CRC microenvironment}

Based on the evidence that also non-neoplastic cells heavily influences cancer progression, therapies targeting the host compartment of tumors have started to be exploited and applied in the clinic.

In particular, great efforts have been made to develop novel therapies targeting CAFs. For example, as mentioned above, CAFs express a membrane-bound serine protease called fibroblast activation protein $\alpha$ (FAP) that is not detected in normal fibroblasts, and whose expression has been associated with an overall poorer prognosis in CRC. FAP is mainly localized in the stroma adjacent to tumor cells but not in the stroma of normal tissue, making it a very attractive candidate for tumor-targeted therapies. However, several studies targeting FAP with a humanized monoclonal antibody (sibrotuzumab) failed to produce clinical benefits in $\mathrm{CRC}^{59}$.

Advances in understanding the role of transforming growth factor (TGF)- $\beta$ in tumorigenesis have led to the development of TGF- $\beta$ inhibitors for cancer treatment. Three platforms of TGF- $\beta$ inhibitors have evolved: antisense oligonucleotides, monoclonal antibodies and small 
molecules ${ }^{60}$. In particular, for CRC treatment a TGF- $\beta 1$-specific phosphorothioate antisense (AP-11014) and TGF- $\beta 2$ inhibitors (Trabedersen, AP-12009) have been proposed ${ }^{61}$.

Several studies have shown the potential of targeting TGF- $\beta$ signaling and, despite earlier predictions of severe toxicity, neutralizing antibodies to TGF- $\beta$ have been well tolerated and have potent antimetastatic activity ${ }^{62}$.

Recently bispecific antibodies (bsAbs) have been introduced to stimulate effector cells to direct their cytotoxic effects against tumor cells. For example, bsAbs comprising a first antigen binding site specific for Death Receptor 5 (DR5) and a second antigen binding site specific for FAP have been introduced for CRC treatment have shown the capacity to strongly induce apoptosis in tumor cells in patient-derived xenograft models ${ }^{63}$.

Immunotherapy has offered promising results in CRC focusing on inducing efficient and specific cytotoxic responses mediated by CD8 $\mathrm{T}$ cells avoiding the side effects of chemotherapeutic drugs. In particular recently several approaches have been explored including checkpoint inhibitors ${ }^{64}$.

$\mathrm{T}$ cells mediated immunity is strongly controlled by a balance system regulated by many stimulatory and inhibitory proteins. In particular, negative regulators of the immune system called immune checkpoints play a key role in inhibiting the immune response. These molecules are expressed on activated $\mathrm{T}$ cells, and upon binding their corresponding ligands, expressed on antigen presenting cells or tumor cells they suppress $\mathrm{T}$ cell activation. Thus, recent studies focused on the use of checkpoints-inhibiting agents (mainly PD-1/PD-L1 and CTLA4) for CRC therapies to interrupt the inhibition of the immune signal against tumors and restore the efficient immune response ${ }^{65,66}$. In particular recent effort has been made to test an-anti-CTLA4 antibody (Ipilimumab) for CRC treatment ${ }^{66}$. Ipilimumab have been already approved for metastatic melanoma treatment however it fails to demonstrate a similar success in CRC. Anti PD-1-mAb (nivolumab and pembrolizuman) have been approved for several type of cancers such as renal carcinoma, non-small cell lung carcinoma (NSCLC), 
head and neck squamous cell carcinoma (HNSCC) and uretherial cancers however it resulted in a low response rate in CRC ${ }^{65}$. Importantly, checkpoints inhibitors resulted in improved antitumor response in MSI-H cancers. The MSI-H are characterized by and higher $\mathrm{T}$ cells density as compared to MSS. However, tumor cells are not eliminated by the immune system due to the upregulation of inhibitory checkpoints. These data indicate that MSI-H cancers might be good candidates for checkpoint immunotherapy ${ }^{67}$.

Finally, a novel CEA IgG-based T-cell bispecific (TCB) antibody is currently in phase I clinical trials for the treatment of CEA-expressing solid tumors. CEA-TCB antibody recognizes CEA and CD3e via inducing $\mathrm{T}$ cell-mediated killing of CEA over-expressing tumors while sparing primary cells with low CEA expression ${ }^{68}$

\subsection{Personalized and precision medicine for CRC}

Recently, many efforts have been made to tailor anticancer treatment to the individual patient's characteristics.

In December 2015, the EU Health Ministers in their "Council conclusions on personalized medicine for patients, defined the personalized medicine as a "medical model using characterization of individuals' phenotypes and genotypes (e.g. molecular profiling, medical imaging, lifestyle data) for tailoring the right therapeutic strategy for the right person at the right time, and/or to determine the predisposition to disease and/or to deliver timely and targeted prevention."

The terms precision and personalized medicine have often been used interchangeably. However, the personalized medicine term imply that unique treatments can be designed for each individual whereas precision medicine approaches may lead to non-personalized interventions that can be used population-wide.

Precision medicine requires a deep understanding of the genetic mutations in addition to the development of new targeted therapies that matches those genetic changes. 
CRC have particularly benefit from the recent advances in precision medicine that aims to predict patient's response to anticancer treatments using novel molecular tools.

Genetic tests, such as RAS and MSI tests, are now available to assess risk, enhance prognosis and predict treatment response. In addition to tumor tissue genotyping, analysis of tumor DNA from liquid biopsy has been shown to provide a rapid test able to reflect the mutation status of tumor tissue. Additional tests intent to predict the toxicity of a specific anticancer treatment. For example, Theraguide 5-FU (Myriad Laboratories) is able to predict the 5-FU toxicity based on detection of the mutations in two genes involved in fluoropyrimidine metabolism. These tests might be useful to adjust the dose or to choose alternative therapeutic approaches. Finally, the advent of precision medicine, despite quite new for CRC medicine, is gradually becoming an integral part of the process of care by providing important tools to predict treatment outcomes.

However, a pre-requisite for the development of tailored treatments is the possibility to predict patient responsiveness. Systems able to predict the individual response to the several anticancer treatments now available are still missing. 


\section{CRC models to predict drug responsiveness}

\subsection{Two-dimensional (2D) CRC models}

A huge effort has been recently made to develop adequate in vitro models which may reliably predict tumor responsiveness to therapeutic treatments.

Cell lines culture in dimensional monolayer have played a key role in the development of new anticancer drugs. In particular, Since the late 1980s, The US National Cancer Institute (NCI) 60 human tumor cell line anticancer drug screen (NCI60), a panel of cancer cell lines representing nine distinct tumor including colon cancer, has been used as in vitro drugdiscovery tool. The most widely used cellular model for CRC has been the Caco- 2 cell line, obtained from a human colon adenocarcinoma, reaching over 13,590 references in the PubMed database in April 2016.

Despite being a powerful tool, cell lines do not maintain functional features of primary cells. Indeed, they may show different phenotypes, native functions and responsiveness to stimuli as compared with primary cells. Moreover, serial passage of cell lines can further cause genotypic and phenotypic modification.

Furthermore, since almost all cells in the in vivo environment are surrounded by other cells and ECM in a three-dimensional (3D) fashion, 2D cell culture does not adequately take into account the natural 3D environment of cells including the oxygen, nutrients and metabolites distribution, 3D cell-cell and cell-matrix contact, which is well recognized to influence cell structure, adhesion, mechanotransduction and cell functions.

Currently, in drug discovery, the standard procedure of screening compounds starts with the 2D cell culture-based tests, followed by animal model tests, to clinical trials.

Many of the drugs fail during clinical trials, especially during phase III ${ }^{69}$, which is the most expensive phase of clinical development, largely due to the lack of clinical efficacy and/or unacceptable toxicity. A portion of these failures is attributed to data collected from the 2D 
monolayer culture and in vivo tests in which the cellular response to $\operatorname{drug}(\mathrm{s})$ is altered due to their unnatural microenvironment. Indeed, many experimental animals have compromised immune systems and do not offer the same stroma-tumor interaction as humans, which prevents the efficient translation of novel research to clinical settings ${ }^{70}$. Obtaining concordance between animal models and clinical trials still remains challenging (only $8 \%$ of concordant results) ${ }^{71}$.

These limitations have encouraged the emergence of many 3D methods to bridge the gap between in vitro and in vivo. 3D models offer a system to better translate the complex pathophysiological features of the TME in vitro. The ideal 3D model would eliminate the differences related to species that are usually encountered, allowing drug testing directly on human models.

\subsection{Cell lines-based 3D models}

Extensive evidence demonstrates that cells cultured in 3D mimic in vivo tumor conditions more closely than standard 2D culture with respect to cell morphology and organization, cell heterogeneity, protein and gene expression patterns, cell-cell and cell-matrix contact ${ }^{72}$. Therefore, to date numerous $3 \mathrm{D}$ models have been developed to recreate in culture the complex 3D architecture of the CRC ${ }^{73,74}$. Multicellular tumor spheroids are very small tridimensional cellular structures ${ }^{75}$ which are generated in suspensions on an acellular 3D scaffold or by dispersing cells in a liquid matrix followed by solidification or polymerization (Figure I.5). 


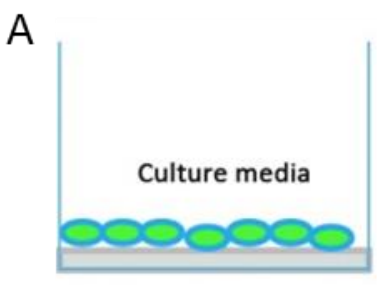

2D monolayer

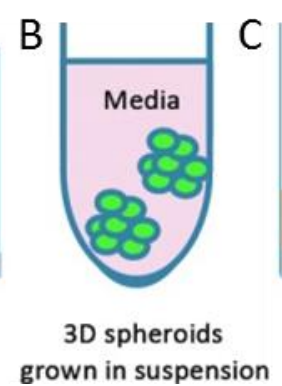

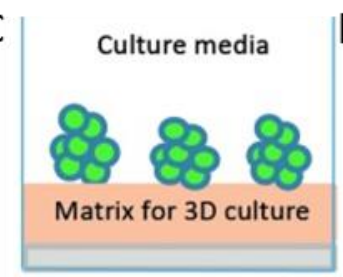

3D spheroids grown on matrix

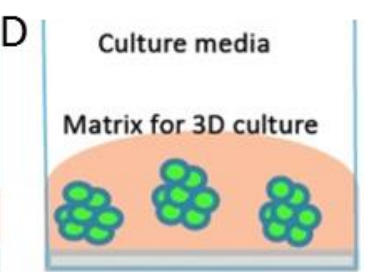

3D spheroids grown within matrix

Figure I.5 - Bidimensional (2D) and multicellular spheroids cultures. Schematic diagrams of the 2D monolayer cell culture (A), scaffold-free cell spheroids in suspension (B) and $3 D$ cell culture systems: cell spheroids grown on matrix $(C)$, cells embedded within matrix $(D)$. Adapted from Edmondson R. et al, $2014^{70}$

Commonly used scaffold/matrix materials used for the generation of multicellular spheroids include biologically derived scaffold systems, such as Matrigel (BD Matrigel ${ }^{\mathrm{TM}}$, BD Sciences), basement membrane extract (Cultrex ${ }^{\circledR}$ BME; Trevigen) or hyaluronic acid-based scaffolds, and synthetic-based materials such as Polyethylene glycol (PEG), polyvinyl alcohol (PVA), polylactide-co-glycolide (PLG), and polycaprolactone (PLA).

Recently, Piccoli et al. generate an organotypic 3D-bioactive model of CRC by seeding HT29 tumor cells on decellularized human biopsies. This model retained major proteins and soluble factors of the ECM and preserved their biological activity in terms of cell attraction and pathway activation ${ }^{76}$.

Multicellular spheroids including different cell types, in addition to tumor cells, have also been proposed.

Nyga et al. developed a complex 3D CRC model based on the co-culture of HT29 epithelial cell line, fibroblasts and endothelial cells seeded in collagen type I gel whereas in 2016, Nietzer et al, developed a 3D in vitro model of CRC based on the coculture of tumor and stromal cells on a biological scaffold derived from decellularized porcine jejunum (small 
intestine submucosa, SIS) obtained a 3D in vitro CRC able to reflect human tissue-related tumor characteristics ${ }^{77}$.

As compared with the corresponding monolayer cultures, multicellular spheroids preserve the tridimensional structure, and the cell-cell and cell-ECM contact.

Furthermore, they preserve the heterogeneity of in vivo tumor tissues inasmuch as are comprised of cells in different stages, including proliferating, quiescent, apoptotic, hypoxic, and necrotic cells. Indeed, the cells of the external layer of the spheroid are exposed to the medium and therefore are mainly viable and proliferating, whereas, the cells of the core receive less oxygen, growth factors, and nutrients from the medium, and tend to be in a quiescent or hypoxic state (Figure I.6).

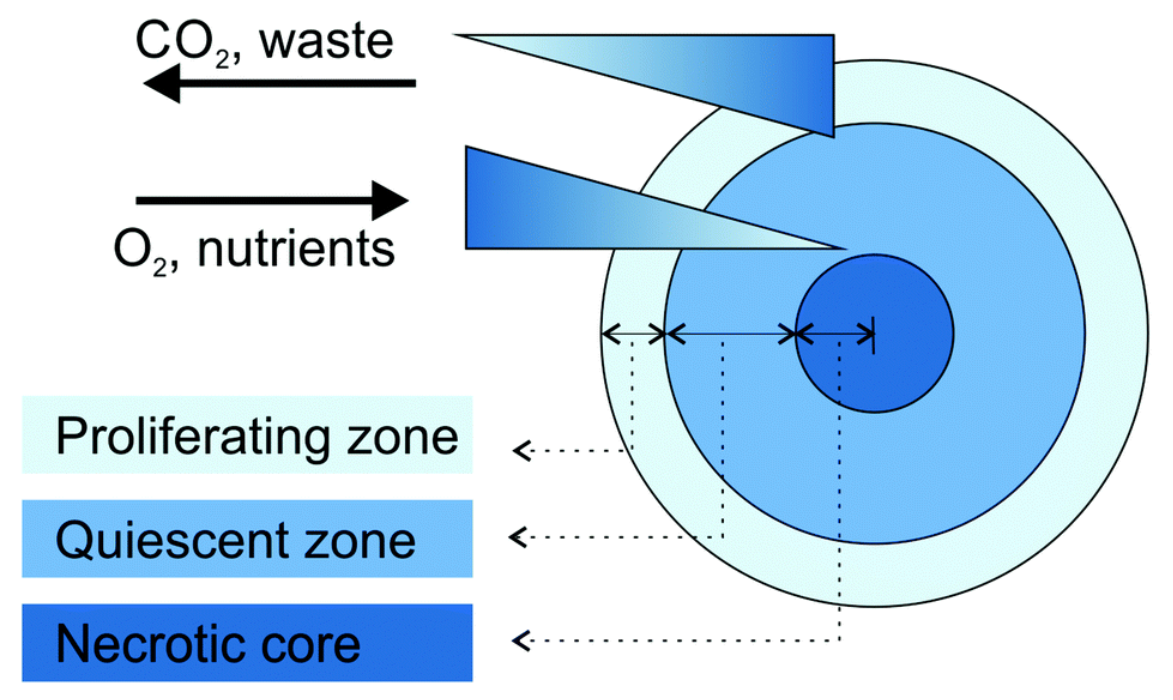

Figure I.6 - Models of oxygenation, nutrition, CO2 removal in a multicellular spheroid.

Cells of the external layer are exposed to higher oxygen concentration and lower waste products as compared with the central region or the core thus presenting higher proliferative state $^{78}$.

In addition, cells cultured in 3D have shown a different proliferation rate as compared to 2D. For example, it has been demonstrated that that a variety of CRC cell lines on Laminin-richextracellular matrix (IrECM) showed a reduced proliferation rate in 3D as compared with 2D cultures ${ }^{70}$. 
Cellular response to anticancer treatments in 3D has been shown to better reflect the in vivo sensitivity as compared to $2 \mathrm{D}$ cultures. In particular, several studies demonstrated that $3 \mathrm{D}$ models are more chemoresistant than 2D cultures. For example, Karlsson et al. ${ }^{79}$ showed that CRC HCT116 cells cultured in 3D spheroids were more resistant to several anticancer treatments (melphalan, 5-FU, oxaliplatin, and irinotecan) than in 2D.

The multicellular resistance can be explained by the poor proliferation rate in the center and core of the spheroid since quiescent cells are not sensitive to most anticancer drugs ${ }^{80}$. In addition, the increased drug resistance in $3 \mathrm{D}$ might be due to limited ability of drugs to penetrate the spheroid and to reach all the tumor cells and to hypoxia, which has been shown to lead to the activation of genes involved in cell survival and drug sensitivity ${ }^{58}$.

\subsection{D models based on primary cells}

It has been widely demonstrated that cell lines, despite maintaining some properties of the original cells, they show clear differences in genetics, epigenetics, and gene expression profiles as compared with primary cells ${ }^{72}$. Thus, recent attempts have been made to develop 3D models from human primary CRC cells.

However, maintenance of primary CRC cells has proved very difficult, possibly due to the insufficient maintenance of cancer stem cells (CSCs), a rare subpopulation of cells with high self-renewal, under serum-based culture conditions conventionally used for cell lines.

Ricci-Vitiani demonstrated that CD133+ CSCs sorted from primary CRC can be maintained in vitro as spheroids structures when cultured with 5\% serum. However, this occured with very variable efficiency ${ }^{81}$. Kondo et al. showed that CRC spheroids from dissociated cell clusters, called "cancer tissue-originated spheroids" (CTOSs), cultured into collagen type I extracellular matrix, could be maintained for long periods (up to 14days) and can be passage (up to 22 times). However, in both the previous cited studies the spheroids culture the 
efficiency was low $(<50 \%)^{82}$. Organoids are 3D stem cell cultures that self-organize into ex vivo 'mini-organs'.

In 2009, Sato et al. proposed a robust method which enabled the production of self-renewing dysplastic intestinal organoids containing intestinal stem cells (ISCs), responsible for CRC expansion in vivo, which could be expanded indefinitely ${ }^{83}$. CRCs crypts were cultured in a solubilized basement membrane (Matrigel) rich in matrix proteins, including laminin and collagen, to mimic the microenvironment of the crypt base in vivo, and supplemented with niche factors (EGF, Noggin, ALK4/5/7 and P38 inhibitor) to support crypt growth ${ }^{84}$.

It has been shown that intestinal crypt organoids are able to accurately predict the apoptotic response to 5-FU in the mouse intestinal epithelium than either of the conventionally used CRC cell lines Caco-2 or MC38 ${ }^{85}$.

Overall, spheroids and organoid cultures resemble the complex spatial morphology of the native tissue and allow cell-cell and cell matrix interactions.

One limitation of organoids and multicellular spheroid models is the limited growth over a long culture time: without blood perfusion, the passive diffusion of nutrient and $\mathrm{O} 2$ into the spheroids becomes insufficient to maintain the functions of the cells in the centre of the spheroids, which then causes significant necrosis when the spheroid diameter is $>600 \mu \mathrm{m}^{86}$ In addition, those models lack the tissue heterogeneity. In fact, organoid cultures preserve the epithelial component but it is not clear whether they can maintain stromal or immune cells. Finally, whether the drug responses in organoid in vitro can predict clinical response, in patients still remains to be addressed. ${ }^{83}$.

Short-term culture (up to three days) of CRC explants in tumor-grade matched matrix has been proposed by Madjumder et al. for the prediction of tumor response to chemotherapy. The system maintains the original tissue architecture and matrix proteins components and has been shown to accurately predict response to tumor cell-targeted therapies ${ }^{87}$. However, 
whether stromal cells and immune cells also survived under these conditions was not assessed.

\subsection{D CRC perfused models}

Recent advances in microfabrication techniques have created a unique opportunity to develop 3D microfluidic platform that more accurately reflect in vivo human biology when compared with two-dimensional flat systems or animal models.

Recent attempts have been made to recapitulate the leaky and tortuous microcirculation of solid tumors. Recently in vitro vascularized microtumors (VMTs) that incorporate human tumor and stromal cell line have been developed. The VMT platform provides a unique model for studying vascularized solid tumors in vitro ${ }^{88}$.

In 2013, Moya et al developed a microphysiological system of CRC organoids obtained from CRC cell line (SW620 and HCT116) and perfused by human microvessels using a pluripotent stem cell (iPSC) technology able to recapitulate the microcirculation of the tumor ${ }^{89}$. More recently, 3D perfused tumor spheroids for anticancer drug testing have been developed starting from CRC DLD-1 cell line. Spheroids in perfusion showed an improved cell viability and increased growth potential compared with spheroids cultured in static condition after over 17 days of culture. Moreover, perfusion provided a more accurate prediction of drug toxicity and efficacy than traditional in vitro tumor models in conventional static culture well plates. In particular, in perfusion, paclitaxel showed a higher cytotoxicity, whereas 5-FU had fewer efficacies as compared to static culture ${ }^{90}$. Recently, studies from our group have shown that culturing CRC cell lines in a perfused bioreactor system previously utilized for the culture of different mesenchymal cell types results in the development of tissue-like-structures efficiently mimicking phenotypes, gene expression profiles and drug resistance patterns observed in cell-line derived xenografts. ${ }^{91}$. 


\subsection{Patient-derived tumor xenograft}

Patient-derived tumor xenograft (PDTX), developed injecting primary tumor cells into an immunodeficient mouse, have been used to predict drug response of individual patient tumors 92. However, tumor uptake rate might be low (ranging from $30 \%$ to $60 \%$ ) and tumor development might require a longer period of time. Also, obtaining a sufficient number of PDTX may require additional "passage" in into other recipient mice. During this time patient's tumor might change the genetic characteristics ${ }^{93}$ and biological behavior ${ }^{73}$. More importantly, PDTX cannot fully mimic the human CRC microenvironment. In fact, over time, the human stromal cells are replaced by the murine stroma, although the exact timing and replacement of human to with murine stromal cells remains unclear ${ }^{94}$. Despite these limitations, there are certain aspects of efficacy and toxicity that will always require evaluation in animal models prior to human clinical trials. In conclusion, despite PDTX represent an attractive system for CRC drug development, the increasing need for personalized medicine will require novel culture system able to more accurately mimic in vivo conditions. Moreover, preclinical studies that utilize the advantages of $3 \mathrm{D}$ culture can greatly improve the understanding of cancer biology, eliminate poor drug candidates, and, most promisingly, reveal new more physiologically-relevant targets that might have been missed in 2D screens. Establishing a 3D model system can save time and money by generating more significantly realistic results. 


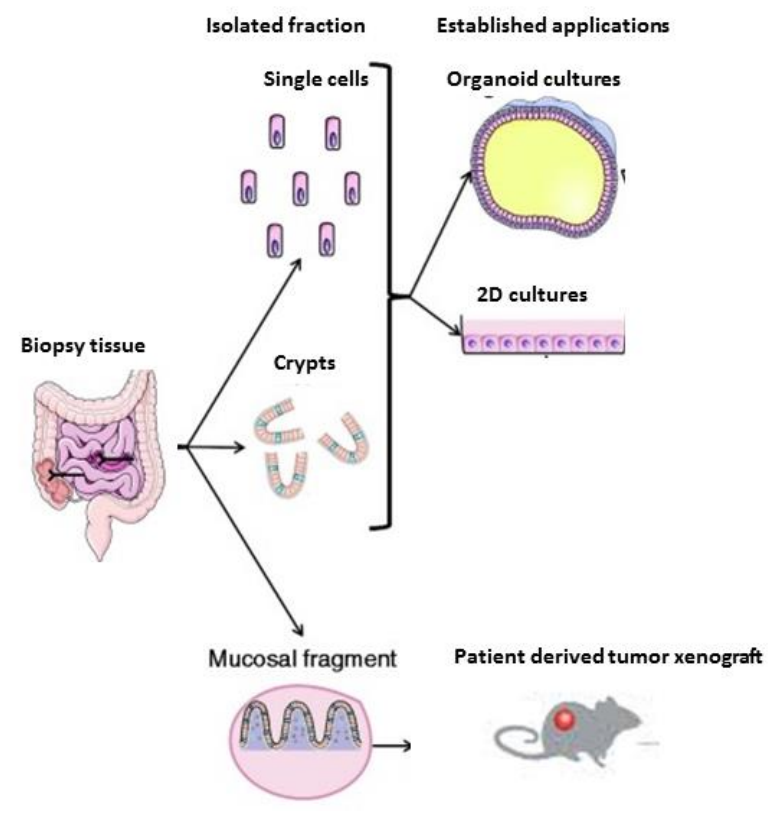

Figure I.7 - 2D and 3D primary cell cultures. ( adapted from Fatehullah et al, 2016 ${ }^{95}$ ) 


\section{CHAPTER II}

AIM OF THE STUDY 
The limited response rate to therapies currently available for patients suffering from CRC urges the development of novel, more effective treatments.

Accumulating evidence indicates that non-transformed cells within the TME also play key roles in the control of tumor progression and response to treatment. However, the possibility to assess their impact on tumor cell responsiveness to novel anti-cancer therapies is hampered by the lack of preclinical systems reliably mirroring the interaction between malignant and non-transformed cells.

Previous studies from our group have addressed the suitability of a perfusion-based bioreactor, previously developed for engineering of cartilage, bone tissues ${ }^{96,97}$ and for tumor engineering purposes ${ }^{91}$. Culture of CRC cells from established cell lines on a collagen scaffold under perfusion resulted in the formation of tumor tissue-like structures whose gene expression and, most importantly, drug sensitivity profiles, were more similar to those of in vivo developed tumors as compared to cells cultured as monolayers or in static 3D conditions $^{91}$.

Capitalizing on these data, during my $\mathrm{PhD}$ studies I have been exploiting the use of the perfusion-based bioreactor for in vitro culture of human primary CRC specimens. In particular, I have addressed the suitability of the perfusion-based bioreactor for:

- The maintenance of the heterogeneity of CRC microenvironment as compared to static cultures

- The assessment of drug responsiveness of primary CRC tissues 


\section{CHAPTER III}

MATERIALS AND METHODS 


\subsection{Tumor sample processing}

Human primary colorectal cancer specimens were obtained from consenting patients undergoing surgical treatment at the University Hospital Basel, St. Claraspital in Basel, and Ospedale Civico of Lugano, all in Switzerland. Use of human samples was approved by local ethical authorities (Ethikkommission Nordwest und Zentralschweiz, study protocol no 2014388). Clinico-pathological characteristics of tumor samples used are listed in Table III.1.

\begin{tabular}{|c|c|c|}
\hline \multicolumn{2}{|c|}{ Features } & \multirow{2}{*}{$\begin{array}{c}\text { Frequency } \mathbf{n}(\%) \\
72(49-87)\end{array}$} \\
\hline Age & Mean (range) & \\
\hline \multirow[t]{2}{*}{ Gender } & Female & $11(58)$ \\
\hline & Male & $8(42)$ \\
\hline \multirow[t]{2}{*}{ Tumor location } & Left-sided & $9(47)$ \\
\hline & Right-sided & $10(53)$ \\
\hline \multirow[t]{3}{*}{ Grade } & G1 & $0(0)$ \\
\hline & G2 & $14(78)$ \\
\hline & G3 & $4(22)$ \\
\hline \multirow[t]{4}{*}{ pT stage } & $\mathrm{T} 1$ & $1(5)$ \\
\hline & $\mathrm{T} 2$ & $5(26)$ \\
\hline & T3 & $9(47)$ \\
\hline & $\mathrm{T} 4$ & $4(21)$ \\
\hline \multirow[t]{3}{*}{ pN stage } & NO & $12(63)$ \\
\hline & N1 & $5(26)$ \\
\hline & $\mathrm{N} 2$ & $2(11)$ \\
\hline \multirow[t]{2}{*}{ Distant metastasis } & Absent & $16(84)$ \\
\hline & Present & $3(16)$ \\
\hline
\end{tabular}

Table III.1. Clinical pathological characteristics of patients included in the study. * 
Tumor specimens were sampled by experienced gastrointestinal pathologists from freshly resected cancer tissues based on macroscopical evaluation, and maintained in Custodiol HTK solution (Dr. Franz Köhler Chemie $\mathrm{GmbH}$ ) at $4^{\circ} \mathrm{C}$ until used. To reduce the risk of contamination due to stool residues, specimens were rinsed in PBS before processing, and incubated in a $10 \%$ Octenisept (Schülke \& Mayr AG) solution for 5 minutes. After additional washings in PBS, tumor specimens were fragmented in $2 \times 2 \times 2 \mathrm{~mm}$ chunks. Three randomized chunks from each sample were immediately embedded in Optimal cutting temperature compound (OCT, Leica Biosystem) and preserved at $-80^{\circ} \mathrm{C}$, as "Fresh" controls. Remaining fragments were weighted and used for cultures.

\subsection{Tumor specimen culture under static conditions}

Three randomized CRC fragments were placed into a culture plate (Sigma) in DMEM/F12 (Gibco) supplemented with 5\% pooled human AB serum (Blood Bank, University Hospital Basel), 2mM Glutamine (GlutaMAX-I, Gibco), 100 $\mu \mathrm{M}$ HEPES (Gibco), 1 $\mu \mathrm{g} / \mathrm{mL}$ Kanamycin

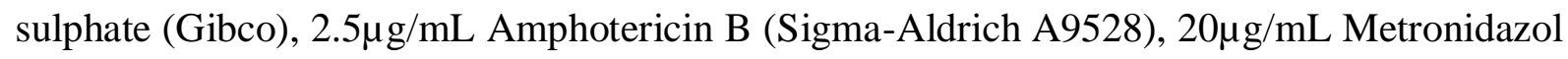

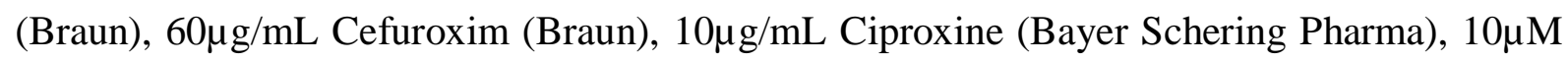

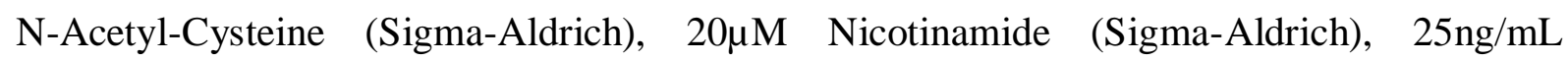
Epidermal Growth Factor (Stem Cell Technologies), 0.1 $\mu \mathrm{g} / \mathrm{mL}$ Prostaglandin E2 (Tocris Bioscience). Three days after, fragments were collected, weighted upon removal of superficial medium by absorbent tissues, and embedded in OCT for subsequent histomorphological evaluation.

\subsection{Tumor specimen culture in bioreactor under perfusion}

Two scaffold discs $(8 \mathrm{~mm}$ diameter $\mathrm{x} 3 \mathrm{~mm}$ ), made from a porous water insoluble partial hydrochloric acid salt of purified bovine corium collagen sponge, known as Ultrafoam Collagen Hemostat (Avitene, Bard), were soaked in culture medium for $1 \mathrm{~h}$ at $37^{\circ} \mathrm{C}$. 
Three randomized CRC fragments were placed between the discs in a sandwich-like configuration. The sandwich was assembled within a ring-shaped plastic holder closed on top and bottom by two EFTE nylon meshes (Fluorotex Sefar, 09-590/47) (Figure III.1).

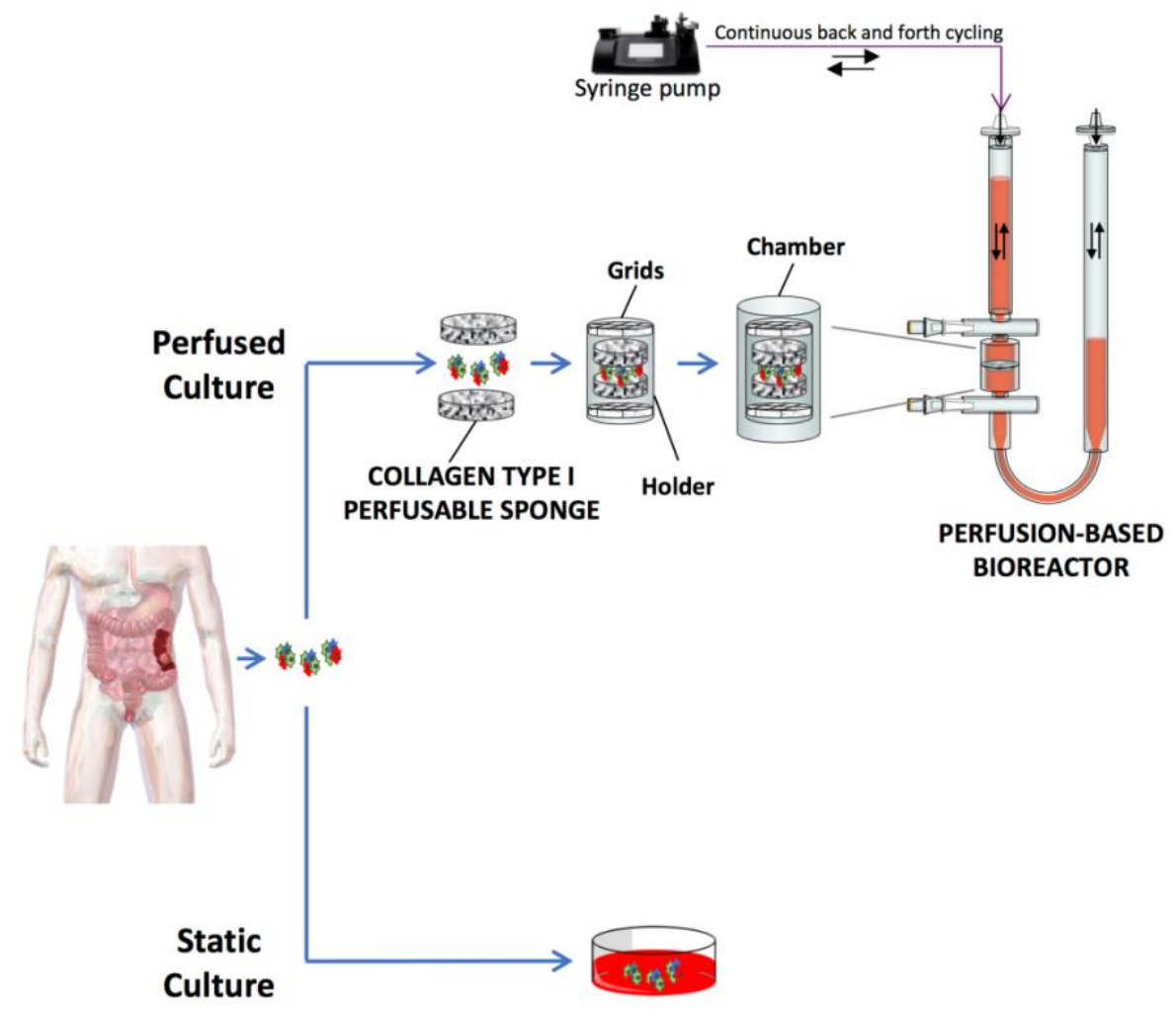

Figure III.1 - Schematic representation of the experimental design. Freshly resected CRC specimens were fragmented in $2 \times 2 \times 2 \mathrm{~mm}$ chunks. For perfused cultures, tumor fragments ( $n=3 /$ bioreactor $)$ were placed between two collagen type I discs within a ring-shaped holder, restrained by two grids on the top and bottom. The holder was then inserted in the bioreactor chamber and subjected to continuous alternate perfusion. For static cultures, tumor chunks $(n=3 /$ plate $)$ were seeded in conventional culture plates. Perfused and static cultures were incubated at $37^{\circ} \mathrm{C}$ for three days.

The scaffold assembly was then placed into the perfusion chamber of a previously described perfusion-based bioreactor ${ }^{98}$ (currently distributed as U-CUP by Cellec Biotek AG) and perfused with the same culture medium used for static cultures. Perfusion flow rate was set at $0.3 \mathrm{~mL} / \mathrm{min}$, corresponding to a superficial velocity of $100 \mu \mathrm{m} / \mathrm{sec}^{99}$, as previously used for the 
generation of normal and tumor tissue-like constructs ${ }^{91,98-101}$. In all experiments, a minimum of two bioreactors including a total of six tumor fragments per condition were used. At the end of the culture, fragments were collected, weighted upon removal of superficial medium by absorbent tissues, and embedded in OCT, for histomorphological evaluation.

\subsection{Histomorphological assessment and immunofluorescence}

Eight-micrometer cryosections were obtained from cryopreserved, OCT embedded, freshly resected tumor chunks, or from fragments maintained in static or perfusion-based cultures. For each specimen 10 sections were cut from $\geq 2$ different levels of the entire tissue block. Slides were fixed with formalin $4 \%$ and stained with Hematoxylin and Eosin (H\&E) or used for immunofluorescence (IF) analysis.

Upon H\&E staining, abundance of epithelial components of neoplastic tissues was semiquantitatively assessed by an experienced gastrointestinal pathologist, based on the evaluation of a minimum of two sections for each specimen and five regions of interest (ROI) per section at 40X magnification using Zeiss Axioskop 2 Plus microscope.

In immunofluorescence studies, epithelial and stromal cells were identified upon staining with (Table III.2) anti-EpCAM mouse mAbs followed by Alexa Fluor 546-labelled goat antimouse polyclonal antibodies, and anti-Vimentin rabbit mAb followed by Alexa Fluor 488labelled goat anti-rabbit polyclonal antibodies. Tumor-infiltrating immune cells were identified by staining with anti-CD45-specific Alexa Fluor 488 labelled mouse mAb. To evaluate proliferating and apoptotic cells, tissue sections were stained with anti-Ki67 488labelled mAb or anti-cleaved Caspase 3 (cC3) rabbit polyclonal antibodies, followed by Alexa Fluor 488-labelled goat anti-rabbit polyclonal antibodies. Nuclei were counterstained by DAPI (Invitrogen). 


\begin{tabular}{c|ccccc}
\multicolumn{1}{c}{ Specificity } & Clone & Host & Company & Code & Dilution \\
\hline EpCAM & VU1D9 & Mouse & Cell Signaling & 2929 & $1: 100$ \\
Alexa Fluor 546 & Polyclonal & Goat & Invitrogen & A-11030 & $1: 100$ \\
Vimentin & D21H3 & Rabbit & Cell Signaling & 5741 & $1: 100$ \\
Alexa Fluor 488 & Polyclonal & Goat & Invitrogen & A-11034 & $1: 100$ \\
CD45 488-labelled & HI30 & Mouse & BioLegend & $14-0459-$ & $1: 100$ \\
& & & & 82 & \\
Ki67 488-labelled & EPR3610 & Rabbit & abcam & ab92742 & $1: 200$ \\
Cleaved Caspase 3 & Polyclonal & Rabbit & Cell Signaling & 9661 & $1: 100$
\end{tabular}

Table III.2 - List of antibodies used in this study.

Images were taken by using IX83 inverted microscope system (Olympus). Numbers of total nuclei or cells positive for Vimentin and $\mathrm{cC} 3$ were analyzed using an automated image quantification program (see below), while the number of CD45 and Ki67+ cells were manually counted. For each specimen five ROI per section were analyzed.

\subsection{Image analysis}

The number of (Vimentin+) stromal and (cC3+) apoptotic cells were quantified using CellProfiler 2.1.1 ${ }^{102}$, an automated image analysis software which can accurately identify cells using advanced algorithms, and whose pipeline can be organized according to specific needs (Figure III.2).

Images of the different components and the nuclei, stained as described above and taken using the IX83 inverted microscope system (Olympus) where collected using separated channels. The images where uploaded in CellProfiler and processed with a well-defined pipeline organized according to the following modules:. 


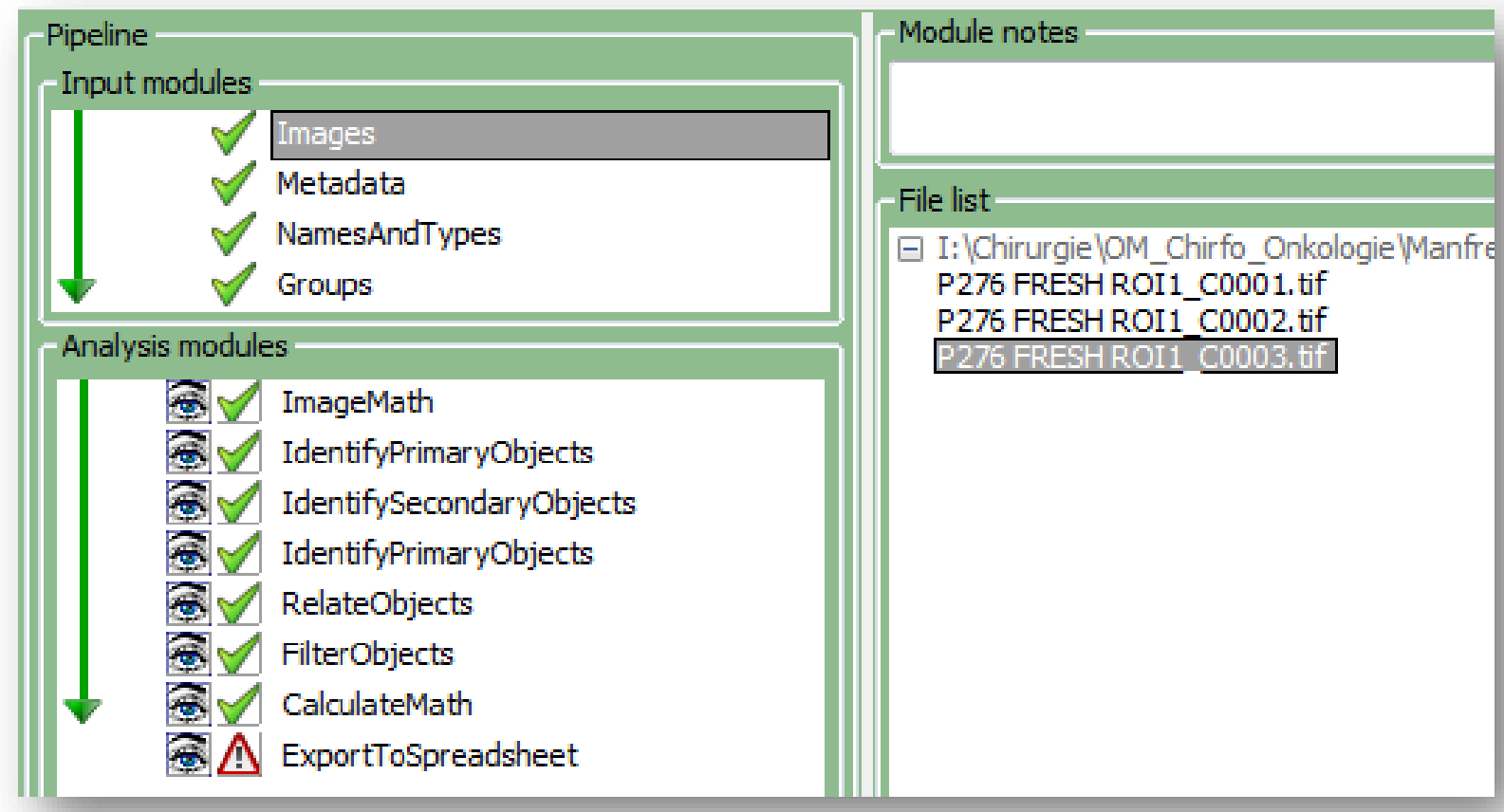

Figure III.2 - CellProfiler interface and pipeline of the work. CellProfiler interface has a pipeline based on several modules that can be modulated according to specific needs. In this figure, the pipeline used for our analysis is depicted. The first module image math is used to subtract the background from the images. The following modules identified the nuclei and cells and finally the last modules calculate the percentage of the objects identified.

- ImageMath - This module performed a subtraction of two different channels of the same image in order to remove the background.

- Identify Primary object (nuclei) - This module, based on a three-step strategy, is used for identification of the nuclei in grayscale images that show bright objects on a dark background. Briefly, first the nuclei were identified based on the typical diameter range of 15, 100 pixels. Then, was determined whether an object was an individual nucleus or two or more clumped nuclei and the object edge was identified based on a thresholding method. Finally, identified objects are either discarded or merged together based on user-defined rules. 
- Identify Secondary Objects - Cell cytoplasm identified based on the distance from the nuclei collected by the previous module.

- Identify Primary object (Vimentin+, cC3+) - The two different channels corresponding to stromal and apoptotic cells were identified using the "identify primary object module", as described above. The dimeter range in pixel is chosen between $\left[1 ; 10^{5}\right]$ pixels.

- The "Related objects" and "filtered objects" modules were used to identify the cells based on the overlap with the nuclei and filter them based on an intensity threshold. Those modules allow discriminating cells from non-specific background.

- $\quad$ Export to Spread Sheet - Measurements were converted to character-delimited text formats and saved to the hard drive in one or several files.

- Calculate Math - Calculate Math took measurements produced by the identification objects modules and calculated the percentage of positive cells.

\subsection{Assessment of functionality of immune and mesenchymal stromal cells}

To assess the functionality of tumor infiltrating lymphocytes, phytohaemagglutinin (PHA, $1 \mu \mathrm{g} / \mathrm{mL}$, Remel Inc.) was added to the culture medium at the beginning of the culture. To evaluate viability and functions of mesenchymal stromal cells, human recombinant IL-17 (10ng/mL, R\&D Systems) was added to the culture medium in the last 24 hours of culture. To evaluate responsiveness to drug treatment, 5-FU $(10 \mu \mathrm{g} / \mathrm{mL})$ was added to culture medium. After three days, culture media were collected and assessed for cytokine content by ELISA.

IL-2, IFN- $\gamma$, and IL-6 release in culture media was assessed by using commercial IL-2 and IFN- $\gamma$ ELISA kits (BD Biosciences), and Human IL-6 Instant ELISA (BioLegend), respectively, according to standard protocols. 


\subsection{Statistical analysis}

Statistical significance of observed differences was tested using Wilcoxon matched-pairs signed rank test. Two-sided $\mathrm{p}$-values were considered significant at $\mathrm{p}$ values $<0.05\left({ }^{*} \mathrm{p} \leq 0.05\right.$; ** $\mathrm{p} \leq 0.01)$. Statistical analysis was performed using GraphPad Prism version 7.0c (GraphPad Software, La Jolla California USA, www.graphpad.com). 


\section{CHAPTER IV}

\section{RESULTS}




\subsection{Culture under perfusion preserves the heterogeneity of CRC microenvironment}

Tumor fragments from freshly resected human CRC specimens were placed between two collagen scaffolds in a sandwich-like configuration and cultured under perfusion in a previously described bioreactor ${ }^{91,98}$ up to three days, a time point used in previous studies ${ }^{87}$ to assess drug responsiveness on human CRC tissues (Figure III.1). Tissue valuable for subsequent analysis was recovered in 23 out of 23 samples.

As static culture controls, in initial experiments tissue samples were embedded in sandwichscaffolds and maintained in bioreactors without perfusion. However, these culture conditions resulted in complete tissue loss, precluding subsequent analysis (data not shown). Therefore, as alternative static condition, tumor fragments were seeded in conventional culture plates

(Figure III.1), a condition allowing the recovery of at least one tumor fragment out of three in all experiments performed $(\mathrm{n}=17)$.

Tissue mass analysis performed before and after culture showed that perfused cultures preserved up to $75 \pm 8 \%$ of tissue weight, as compared to $31 \pm 16 \%$ only in static cultures ( $\mathrm{p}=0.0078$, Figure IV.1A). Accordingly, on histomorphological analysis, perfused tissues maintained higher tissue cellularity, as indicated by total nuclei counts, in comparison to static cultures $(68 \pm 20 \%$ and $40 \pm 23 \%$, respectively, $\mathrm{p}=0.02$, Figure IV.1B). 
A
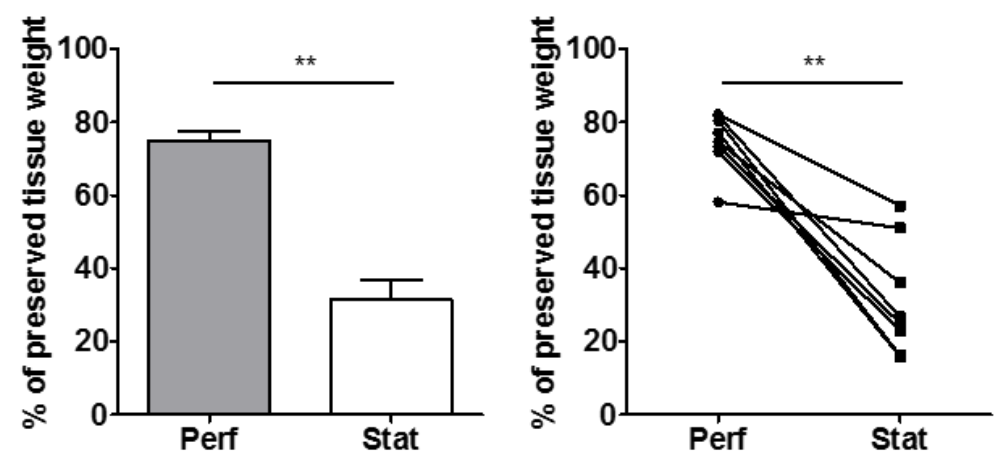

B
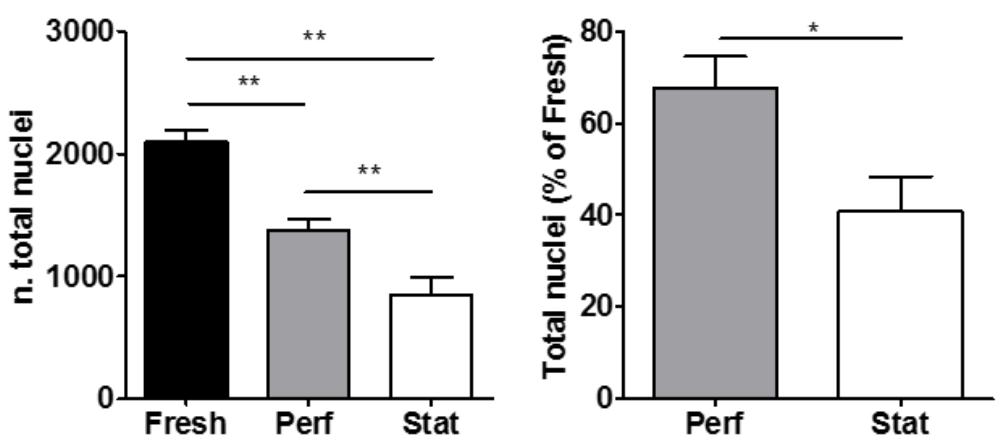

Figure IV.1. Culture under perfusion preserves the heterogeneity of CRC microenvironment. Tumor fragments derived from CRC specimens were weighted, and cultured in perfused bioreactors (Perf) or under static conditions (Stat), as described in Figure III.1. After three days, fragments were collected, weighted, and embedded in OCT for histomorphological evaluation. A. Weight of cultured tumor chunks is shown as percentage of that recorded prior to culture (Fresh). Mean $\pm S D(n=8$, left panel) and individual values (right panel) are shown B. Cultured tissues were stained by DAPI and cell nuclei within five ROI per chunk, identified at 40X magnification, were counted using CellProfiler image analysis software. Cumulative data refer to absolute cell nuclei counts in fresh tissues, chunks cultured under perfusion, or chunks cultured under static conditions (left panel), and cell nuclei counts in chunks cultured under perfusion, or static conditions as related to those of fresh tissues (right panel) ( $n=9)$. Data are expressed as mean $\pm S D$.

Importantly, evaluation of tissue quality and composition showed that original architecture was partially maintained in CRC tissues cultured in the perfused bioreactor, whereas it was completely lost in non-perfused cultures (Figure IV.2). 


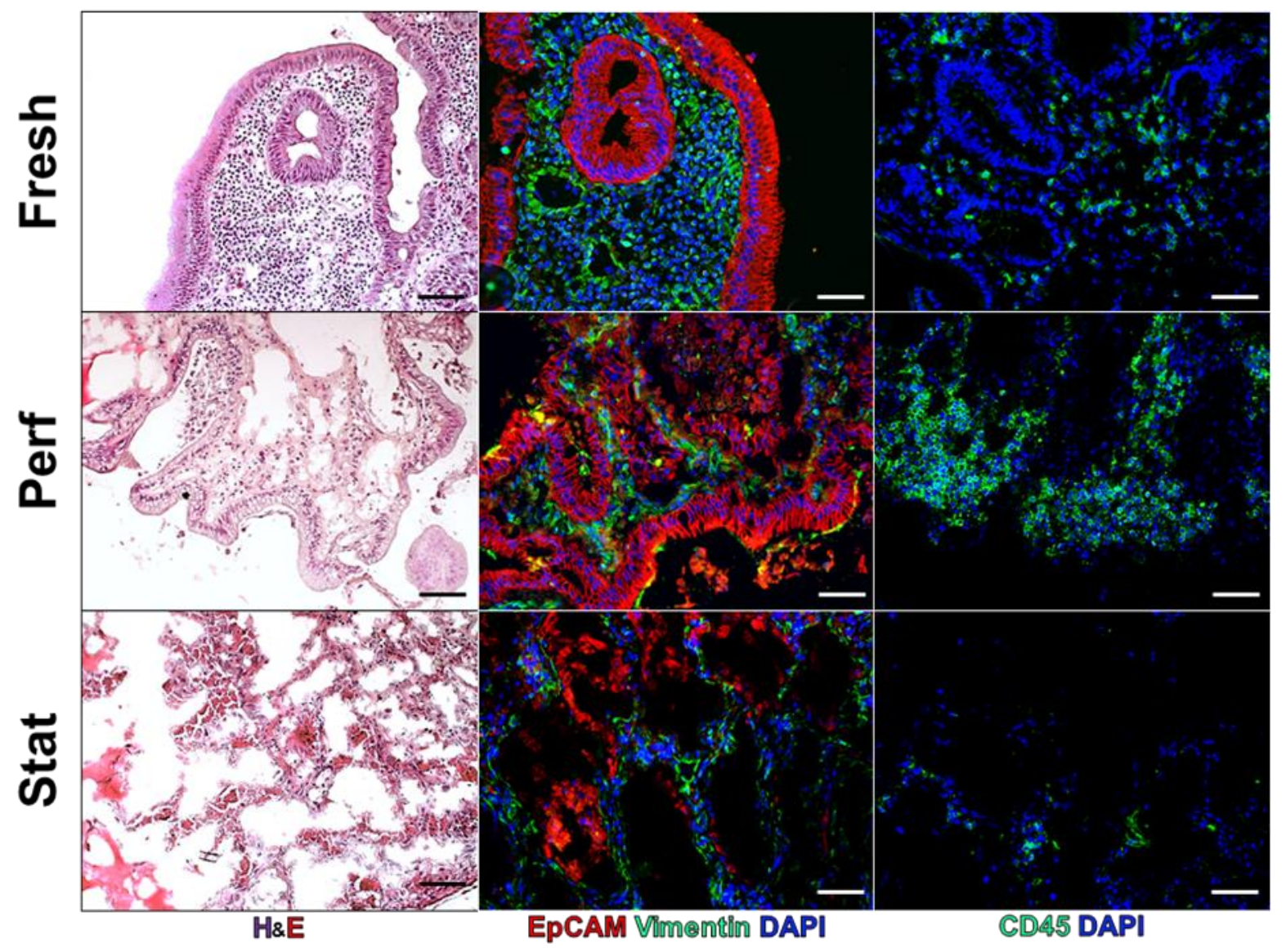

Figure IV.2 - Assessment of TME components in CRC cultures. Fresh tissues (Fresh) or tissues cultured under perfusion (Perf) or static conditions (Stat) were subjected to H\&E staining (left panels) or to IF analysis (middle and right panels), following stainings for EpCAM (red), vimentin (green), CD45 (green), and DAPI (blue). Images from a representative CRC sample were obtained at $20 X$ magnification, scale bar $50 \mu \mathrm{m}$.

In particular, in cultures under perfusion the epithelial component was only partially and not significantly reduced as compared to fresh tissues $(35 \pm 14 \%$ versus $59 \pm 27 \%$, respectively, pvalue $>0.1$. In contrast, tumor tissues cultured under static conditions displayed significantly reductions of epithelial cell fraction $(10 \pm 13 \%, \mathrm{p}=0.022$ versus fresh tissues, $\mathrm{p}=0.016$ versus perfused cultures) (Figure IV.3A). Notably, in four out of seven samples evaluated, the epithelial fraction was completely lost (Figure IV.3A). Numbers of mesenchymal stromal cells, as assessed upon vimentin staining, were also comparable in fresh tissues and tissues cultured under perfusion (out of five ROI: $604 \pm 376$ and $465 \pm 227$ positive cells, respectively, 
$\mathrm{p}>0.3)$. However, they were significantly reduced in static cultures $(128 \pm 102$ positive cells), as compared to both fresh tissues and perfused cultures ( $\mathrm{p}=0.008$ and 0.016 , respectively) (Figure IV.3B). Tumor infiltrating immune cells, identified as CD45+ cells, although being markedly reduced in both culture types, were still clearly detectable in perfused tissues (out of five ROI: $75 \pm 127$ cells versus $173 \pm 145$ in fresh tissues, $p>0.05$ ) whereas they were almost completely lost upon static culture $(9 \pm 9, \mathrm{p}=0.008$ versus fresh tissues, $\mathrm{p}=0.054$ versus perfused cultures, Figure IV.3C). 

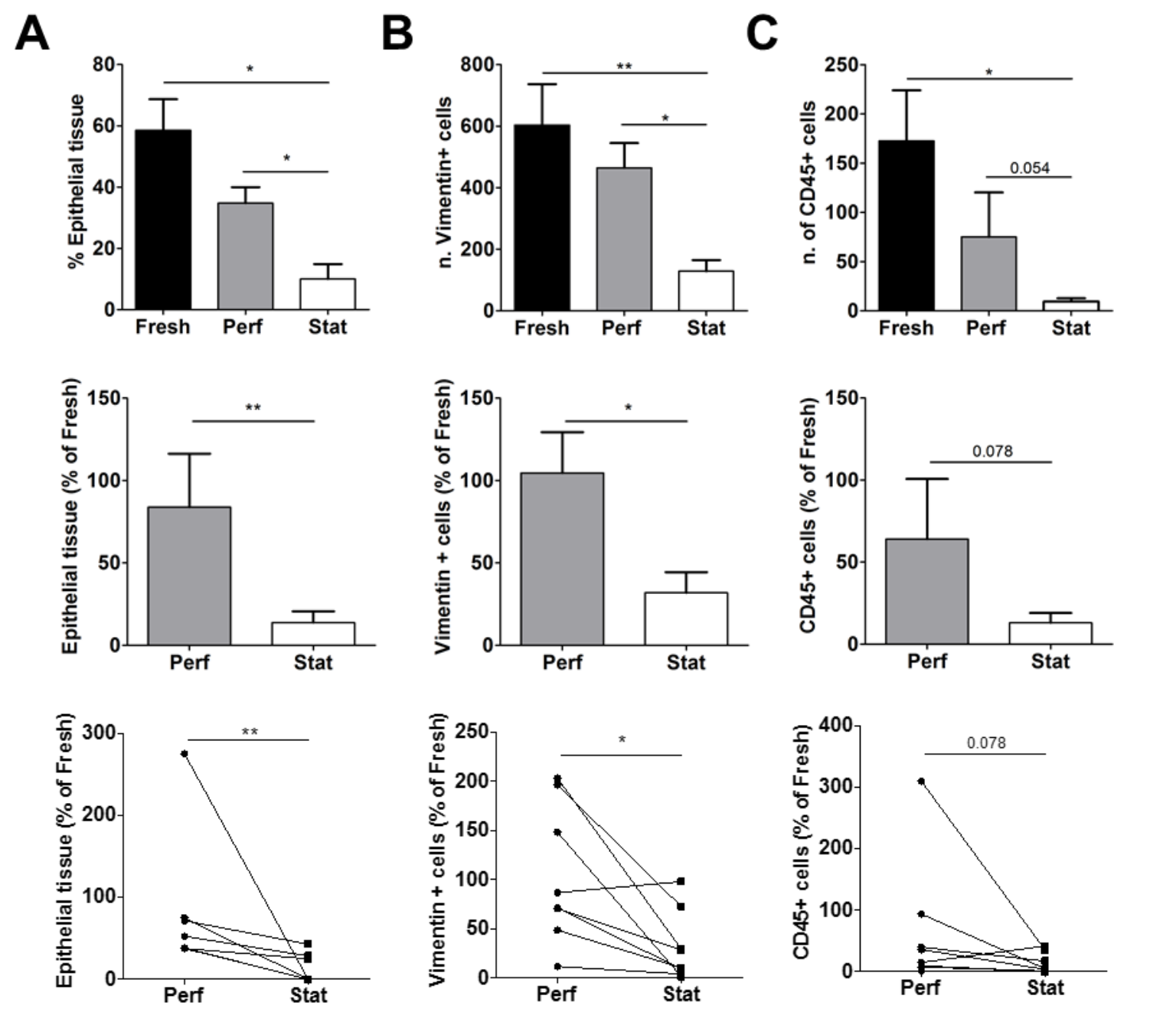

Figure IV.3 - Culture under perfusion preserves the heterogeneity of CRC microenvironment. A. CRC fresh tumor fragments or tissues cultured under perfusion or static conditions were processed and nuclei were stained and counted as detailed in Figure IV.2. Fresh tissues or tissues cultured under perfusion or static condition were subjected to $H \& E$ staining $(A)$ or to IF analysis (B and $C$ ), following stainings for EpCAM, vimentin, CD45 and DAPI. Data refer to percentages of epithelial tissue scored based on H\&E staining (A), or numbers of vimentin+ $(B)$ or CD45+ cells $(C)$ counted out of five ROI per chunk in individual fresh or cultured CRC samples. Statistical significance of differences observed was tested by Wilcoxon signed rank test $(* p<0.05 ; * * p<0.01)$. 
These results indicated that short-term culture of primary tumor fragments under perfusion maintains heterogeneity of CRC microenvironment to significantly higher extents, as compared to static cultures.

\subsection{Perfused cultures better preserved viability and functionality of all CRC cellular components}

To further assess cell viability within cultured tissues, we evaluated proportions of proliferating or apoptotic cells, upon Ki67 or cC3 staining, respectively (Figure IV.4 and Figure IV.5).

In perfused cultures, proliferation and apoptotic rates were comparable to those observed in fresh tissues (Ki67+ cells: $5 \pm 5 \%$ versus $6 \pm 4 \%$; cC $3+$ cells: $7 \pm 7 \%$ versus $5 \pm 4 \%$, respectively, Figure IV.5A and B). In contrast, in static cultures, percentages of proliferating cells were significantly reduced $(1 \pm 1 \%, \mathrm{p}=0.004$ versus both fresh and perfused tissues), whereas those of apoptotic cells were significantly increased $(18 \pm 20, \mathrm{p}=0.047$ versus fresh tissues, Figure IV.5A and B). Therefore, tissues cultured under static conditions displayed markedly and significantly decreased Ki67/cC3 ratios (Figure IV.5C).

Notably, in all conditions proliferating Ki67+cells were only detectable within the epithelial cell fraction. Thus, in tissues cultured under perfusion tumor cells are not only maintained in larger percentages but also in a more active state. 


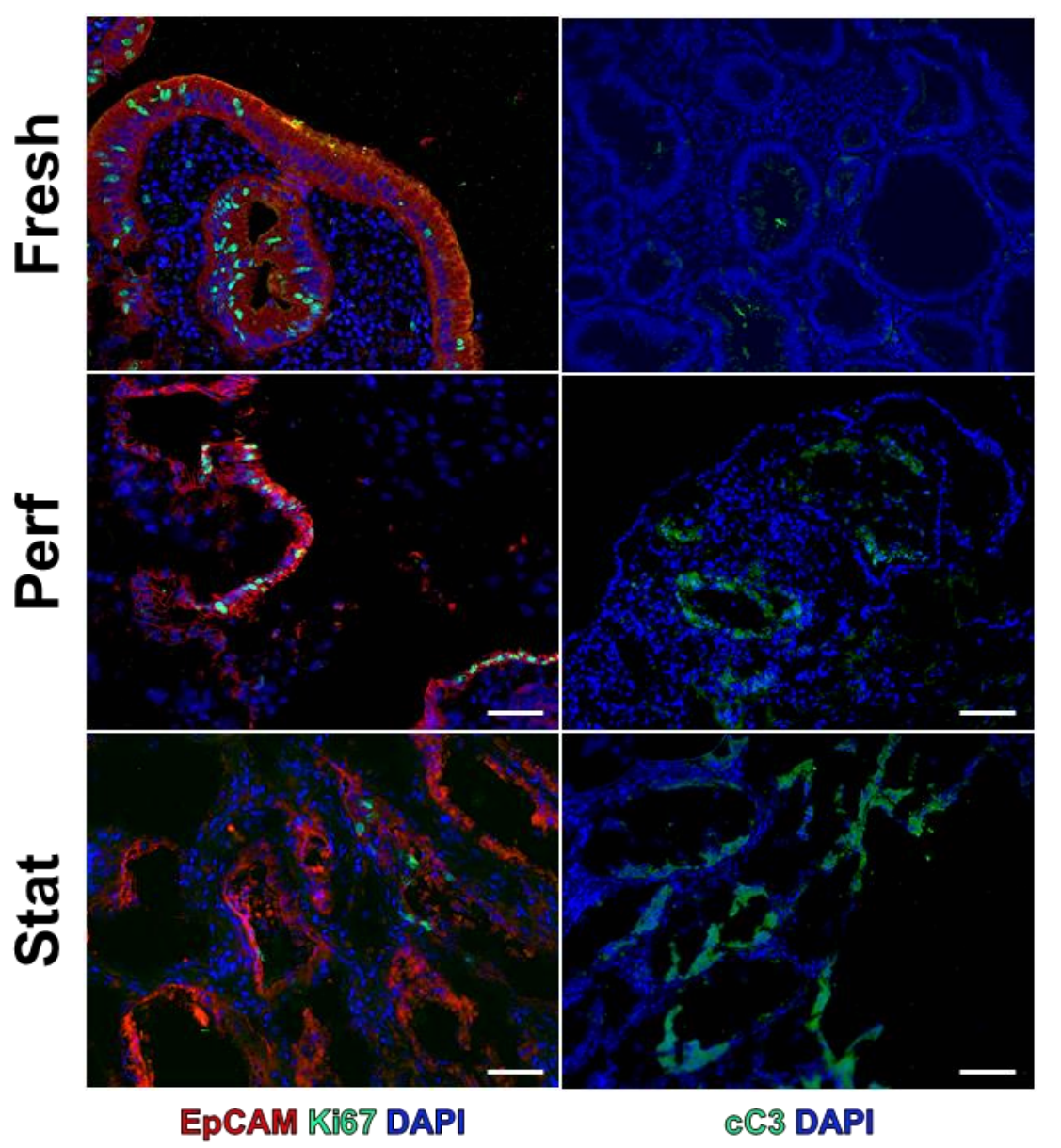

Figure IV.4 - Assessment of tumor cell viability in CRC cultures. CRC fragments freshly resected or cultured for three days in perfused bioreactors (Perf) or under static conditions (Stat), were analyzed by immunofluorescence upon staining for Ki67 (green), EpCAM (red), cC3 (green), and DAPI (blue). Representative images obtained at $20 X$ magnification, scale bar $50 \mu \mathrm{m}$. 
A
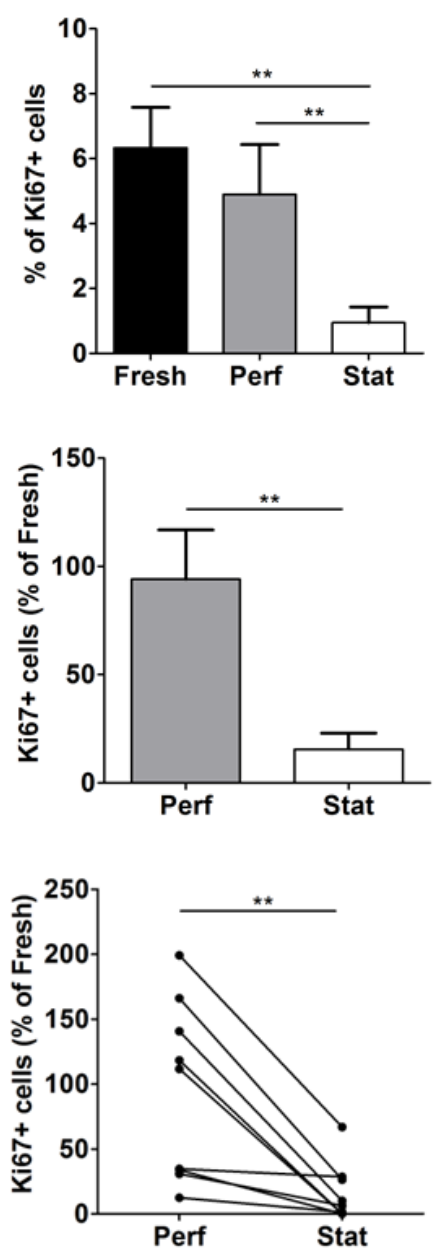

B
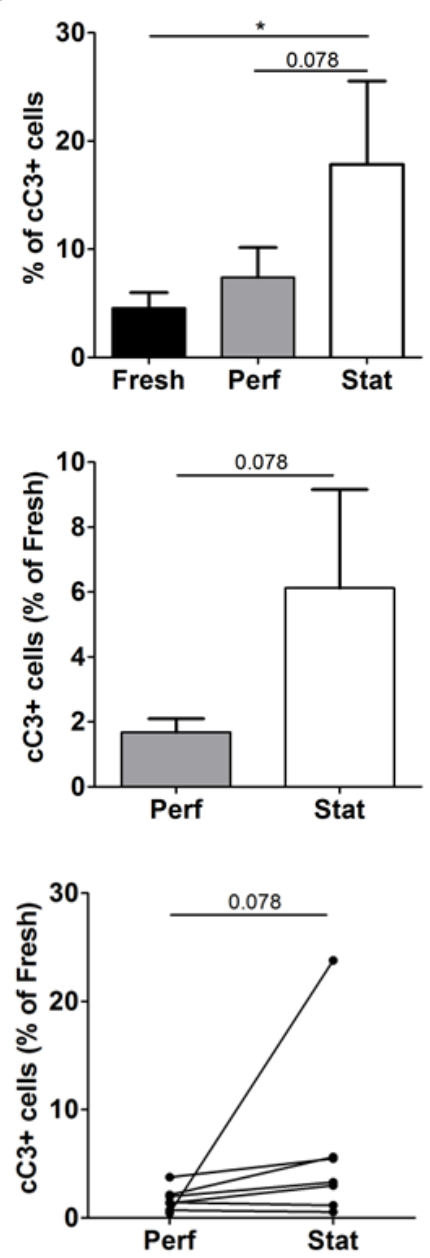
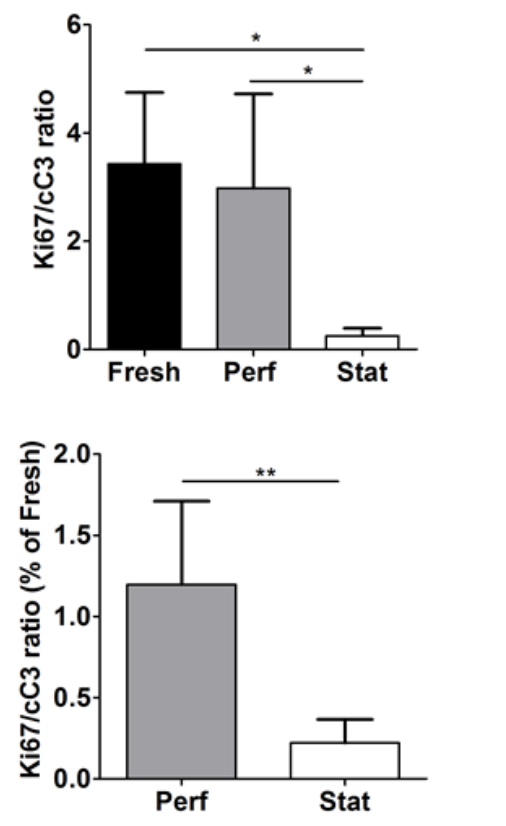

C

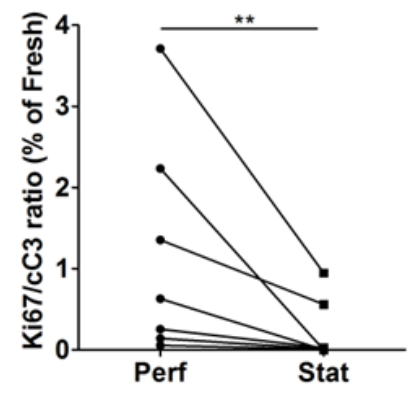

Figure IV.5 - Perfusion allows maintenance of viable and proliferating tumor cells. CRC fragments freshly resected or cultured for three days in perfused bioreactors (Perf) or under static conditions (Stat), were analyzed by immunofluorescence upon staining for Ki67, EpCAM cC3and DAPI. Percentages of Ki67+ $(A, n=9), c C 3+$ cells $(B, n=7)$ as related to numbers of total nuclei, and Ki67/cC3 ratios $(C, n=7)$ with their individual values are shown. Cumulative data are expressed as mean \pm SD. Statistical significance of observed differences was tested by Wilcoxon signed rank test $(* p<0.05 ; * * p<0.01)$.

Next, we investigated functionality of mesenchymal stromal cells by evaluating their IL-6 release, under steady state conditions or upon stimulation with IL17, a pro-inflammatory cytokine promoting IL-6 secretion by stromal cells ${ }^{103}$.

IL-6 release was clearly detectable in media from unstimulated bioreactor cultures. Most 
importantly however, its amount was significantly increased upon IL-17 stimulation $(p=0.03$, Figure IV.6A), thus indicating that stromal cells maintained in perfused cultures were viable and responsive to micro-environmental stimuli. We then assessed viability of tumor infiltrating immune cells cultured under perfusion. In particular, we tested whether infiltrating T lymphocytes, key players of anti-tumor immune responses ${ }^{104,105}$, were still responsive to stimulation. Therefore, we measured release of IL-2 and IFN- $\gamma$ T cell-derived cytokines, upon mitogenic stimulation. As expected, basal levels of T cell-derived cytokines, in particular of IL-2, largely varied between different samples (Figure IV.6B, right panel). Importantly however, they consistently increased upon stimulation (Figure IV.6B), thus suggesting that immune cells within CRC TME may be successfully activated during culture in bioreactors. 
A
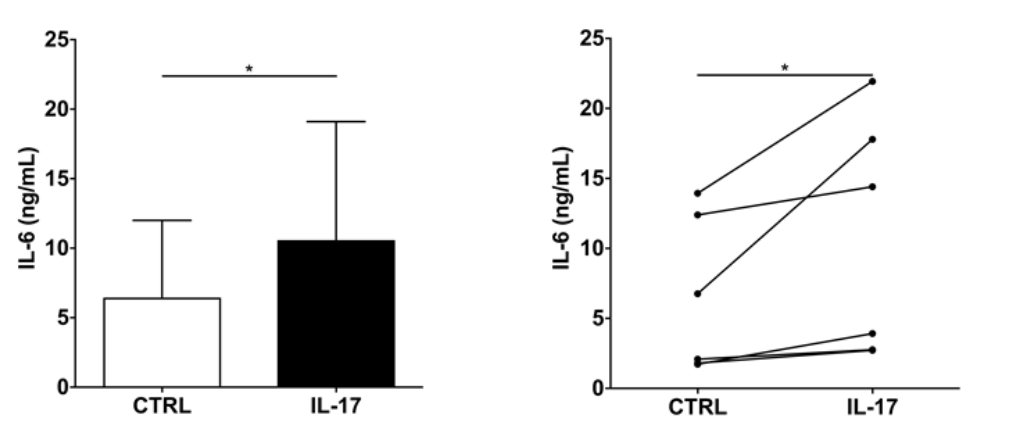

B
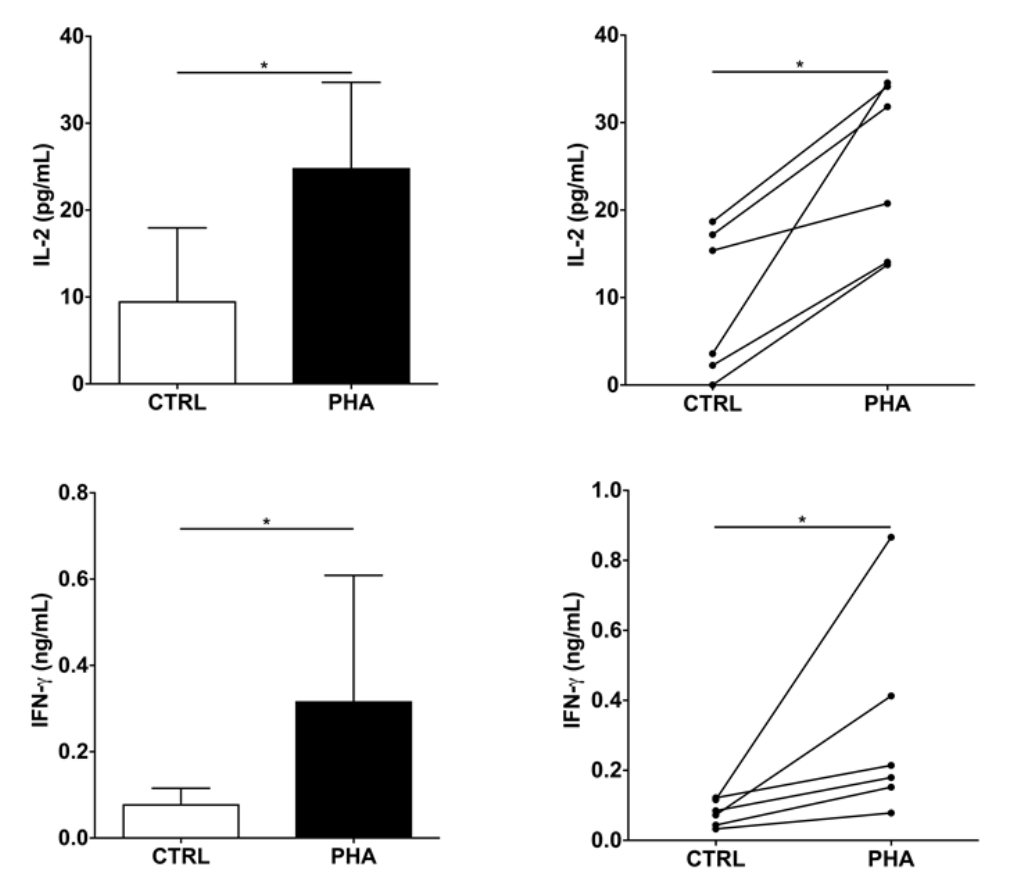

Figure IV.6 - Perfused cultures preserve functionality of tumor-associated stromal and immune cells. CRC fragments were cultured within perfused bioreactors for three days. A. In the last 24 hours of culture medium was supplemented with $I L-17(10 \mathrm{ng} / \mathrm{ml})$ or left unmodified (CTRL). IL-6 release was assessed by ELISA. Cumulative data expressed as mean $\pm S D$ (left panel, $n=6$ ) and data from individual cultures (right panel) are shown. B. Cultures were performed in the absence (CTRL) or presence of PHA $(1 \mu \mathrm{g} / \mathrm{ml})$. Release of IL-2 and IFN- $\gamma$ in culture media was quantified by ELISA. Cumulative data (mean $\pm S D, n=6$, left panels) and data from individual cultures (right panels) are reported. Statistical significance of observed differences was tested by Wilcoxon signed rank test $\left({ }^{*} p<0.05 ; *^{*} p<0.01\right)$. 


\subsection{Bioreactor-based cultures are amenable to test drug responsiveness of primary CRC tissues}

Finally, we evaluated the possibility to exploit bioreactor-based cultures to test responsiveness to drugs in primary CRC tissues. Fragments from six different samples were cultured under perfusion in the presence or absence of 5-FU, and proliferation and apoptosis rates were evaluated (Figure IV.7 and Figure IV.8). Following three days of treatment, an overall significant reduction in percentages of epithelial proliferating cells, and a significant increase in the fraction of apoptotic cells (Figure IV.8A and B) could be observed. Notably however, analysis of individual samples revealed more heterogeneous responses. In particular, two out of six samples showed negligible (P318) or partial (P345) inhibition of proliferation, whereas an additional sample (P343) displayed a cytostatic response only, with a dramatic reduction in proliferation, but no increase in the percentage of apoptotic cells (Figure IV.8C).

Thus, bioreactor-based cultures appear to be suitable to assess patient-specific drug responsiveness in primary $\mathrm{CRC}$ within a short time frame. 


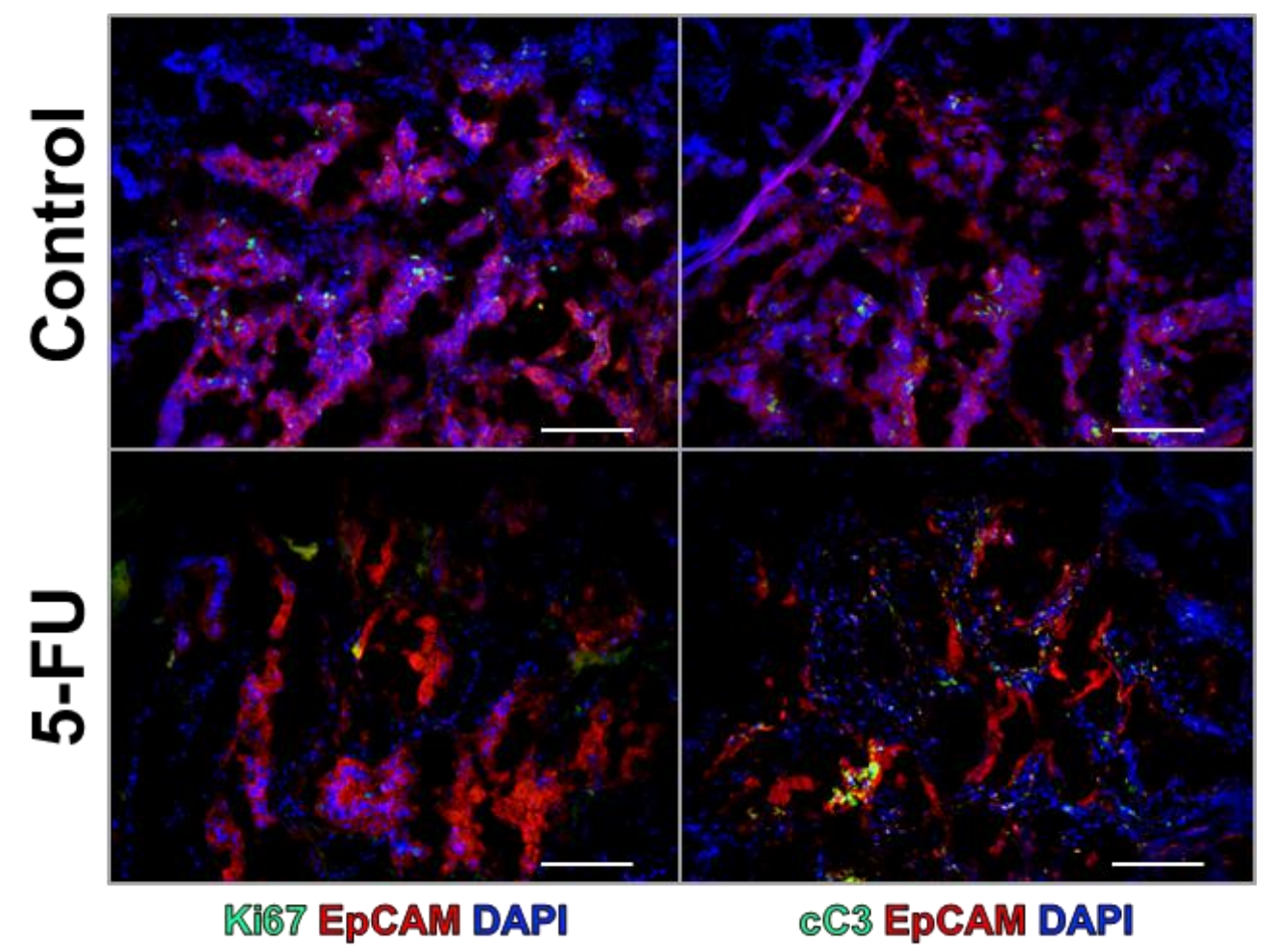

Figure IV.7 - Evaluation of drug responsiveness of primary CRC tissues maintained under perfusion. CRC fragments were cultured within perfused bioreactors for 3 days in the absence (CTRL) or presence of 5-FU $(10 \mu \mathrm{g} / \mathrm{ml})$. Representative IF analysis (magnification 20X, scale bar $50 \mu \mathrm{m}$ ) upon Ki67 or cC3 (green), EpCAM (red), and DAPI (blue) staining. 

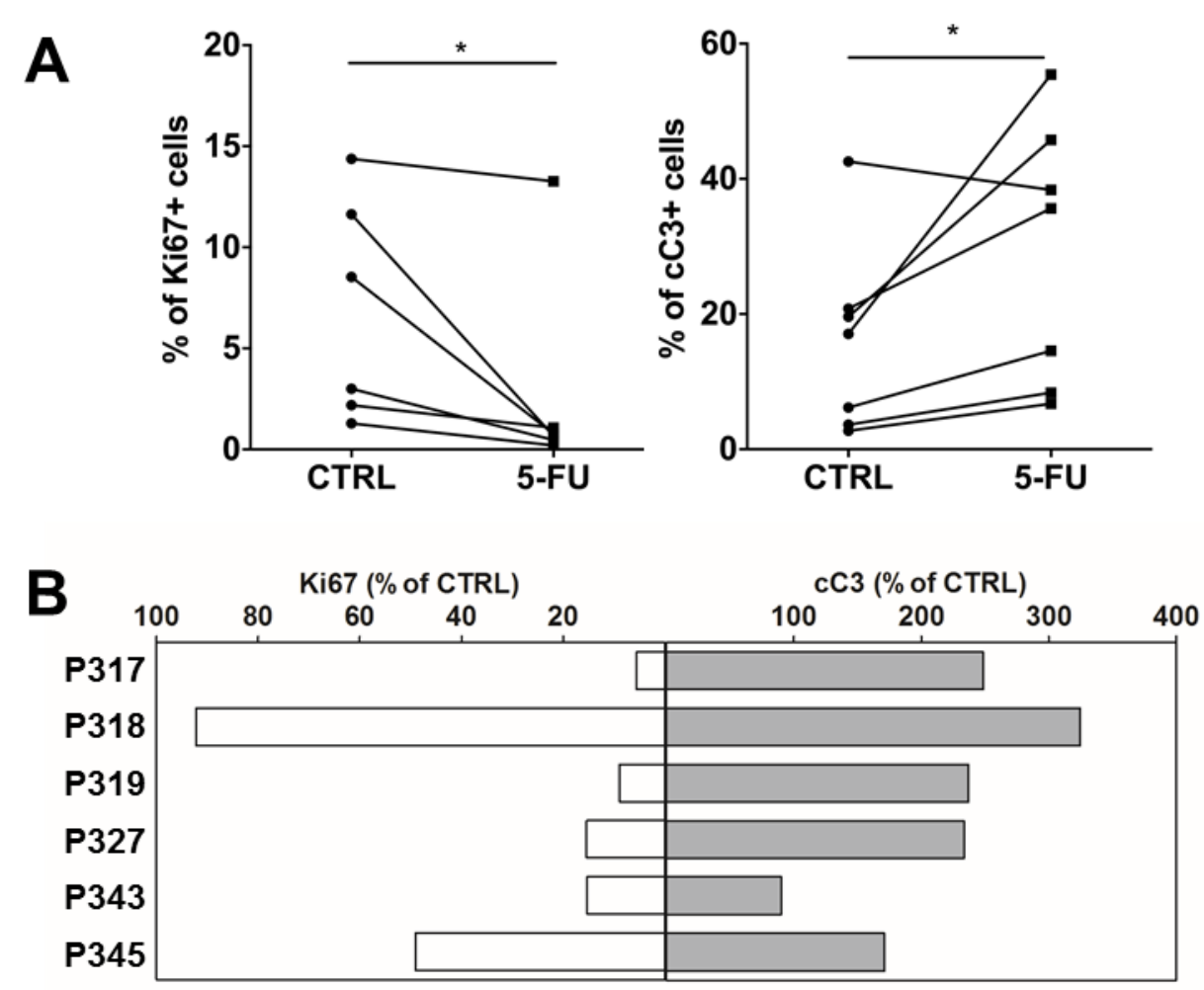

Figure IV.8 - Bioreactor-based cultures are amenable to test drug responsiveness of primary CRC tissues. CRC fragments were cultured within perfused bioreactors for 3 days in the absence $(C T R L)$ or presence of $5-F U(10 \mu \mathrm{g} / \mathrm{ml})$. A. Percentages of Ki67+ (left, $n=6)$ or cC3+ cells (right, $n=7)$. B. Percentages of Ki67+ and cC3+ cells in cultures supplemented with 5-FU relative to untreated tissues in individual samples. 
CHAPTER V

DISCUSSION 
In the last decade, a major effort has addressed the development of tailored treatments targeting specific molecular alterations of cancer cells. However, in the era of "personalized medicine" a major challenge is still represented by the lack of adequate in vitro models predicting responsiveness to specific treatments, and guiding therapeutic decisions. This is particularly important for the management of CRC, a leading cause of cancer-related death, for which success rates of conventional chemotherapeutic regimens are limited, whereas alternative therapeutic approaches may be preferable.

In this work, we have shown that culture of freshly resected human CRC specimens in a bioreactor under perfusion results in maintenance of viable whole tumor tissues, including cancer cells together with mesenchymal stromal cells and substantial fractions of immune cells, for a period reliably allowing assessment of tumor drug responsiveness.

Tumor cells cultured under perfusion displayed an almost intact structure, as compared to the original tumors, and were viable and proliferating. In addition, stromal cells were maintained in proportions similar to those of original tumors and fully viable, as indicated by responsiveness to micro-environmental stimuli, such as IL-17. Furthermore, immune cells were also partially preserved, and were capable of releasing effector cytokines upon activation.

In contrast, in cultures performed under static conditions, fewer viable tumor and stromal cells were preserved, whereas immune cells were completely lost.

Importantly, perfusion-based cultures proved suitable for testing the sensitivity of primary tumor cells to chemotherapies of current use in CRC and revealed heterogeneous responses across individual samples. Thus, our system recapitulates key features of CRC TME and may allow the assessment of tumor response to treatment in a more reliable, patient-specific context.

Established cell lines have long been recognized to substantially differ from primary tumor cells and to fail to mimic the complexity of tumor microenvironment ${ }^{106}$. Therefore, 
innovative culture systems relying on the use of primary tumor cells have been proposed. In particular, regarding CRC, multicellular tumor spheroids and organoids have been developed. Single CD133+ cells sorted from CRC specimens were shown to be able to expand in vitro in tridimensional spheroid-like structures ${ }^{107}$. However, generation of CRC-derived spheres requires digestion of tumor tissues and pre-sorting of CD133+ cells. Furthermore, sphere formation in CRC only occurs in a limited fraction of samples ${ }^{108}$.

Clevers and collaborators have pioneered the establishment of techniques reproducing in vitro "organoids" starting from cell clusters isolated from crypts derived from human normal or neoplastic intestinal epithelium ${ }^{109}$. Organoid formation from primary CRC is highly reproducible and is suitable for high-throughput drug screening. Similarly, CRC tissueoriginated spheroids preserving cell-cell contact between primary tumor cells have been shown to expand with high success rates and to be amenable to drug testing ${ }^{82,110}$.

However, the formation of both spheroids and organoids relies on the generation of new tumor tissues starting from few putative stem cells. Thus, it requires long culture periods and the ability of newly generated structures to mirror original tumor tissue features is questionable.

Most importantly, these culture systems, while allowing expansion of epithelial tumor cells, fail to preserve mesenchymal and immune cell TME components, which critically influence tumor growth and responsiveness to treatment in CRC. Thus, they do not allow the assessment of the responsiveness of cancerous cells to anti-cancer compounds within a context similar to that of in vivo tumors, and in particular to immunotherapeutic strategies.

Short-term culture (up to 3 days) of CRC explants in tumor grade-matched matrix has been proposed as a platform for the prediction of tumor response to chemotherapy ${ }^{87}$. This system certainly represents a major advance in primary tumor culture, inasmuch as it maintains the original tissue architecture and matrix protein components. Indeed, it has been shown to predict response to tumor cell-targeted therapies with high sensitivity and specificity. 
However, it remains unclear whether this system, where cultures are performed under static conditions, also preserves tumor-associated mesenchymal cells and infiltrating immune cells, thus allowing testing of therapies targeting tumor-associated stroma.

Our work shows that culture of human CRC tissues under perfusion preserves viable tumor cells for a period of time (3 days) comparable to that of the above described study. Most importantly, CRC-associated mesenchymal and immune cells, possibly modulating sensitivity of tumor cells to drugs, are also maintained in a viable and active state.

The precise contribution of perfusion flow to maintenance of tumor and tumor-associated cells remains to be fully clarified. The continuous direct perfusion allows removal of cell waste products and maintenance of nutrient levels throughout the constructs, which is in turn promoting cellular proliferation and viability ${ }^{91,99}$. Beyond reduction of mass transfer limitations, the flow-associated shear stress has been reported to facilitate the maintenance of stem cell status and prevent spontaneous differentiation ${ }^{111}$. It may thus be speculated that cancer cells with stem cell-like features, including self-renewal ability, are preferentially maintained within perfused cultures due to the perfusion-driven mechanical signal, mimicking interstitial fluid flow.

In conclusion, the aim of this work was the exploitation of a perfusion-based bioreactor for maintenance of human CRC tissues. Thanks to its capacity to maintain TME heterogeneity, our system may allow personalized drug testing within a more physiological context. 


\section{CHAPTER VI}

\section{FUTURE PERSPECTIVES}




\subsection{System's optimization and limitations}

A number of features of the culture condition still need to be optimized, including culture period, scaffold type, flow rate and clinical response.

\subsubsection{Culture period}

Indeed, our results showed that our system maintain alive up to three days all the cellular components of the native tissues. The possibility to extent the culture period to later time points will allow the test of tumor responsiveness to emerging immunotherapies that might required more time to be effective. In particular, we are now evaluating the possibility to extent the culture to later time points. In preliminary experiments CRC samples were cultured in the perfusion-based bioreactor for 3,5, 7 and 10 days. Over time the tissue mass, in perfused cultures, appear to be maintained whereas it is progressively reduced in static conditions (Figure VI.1).
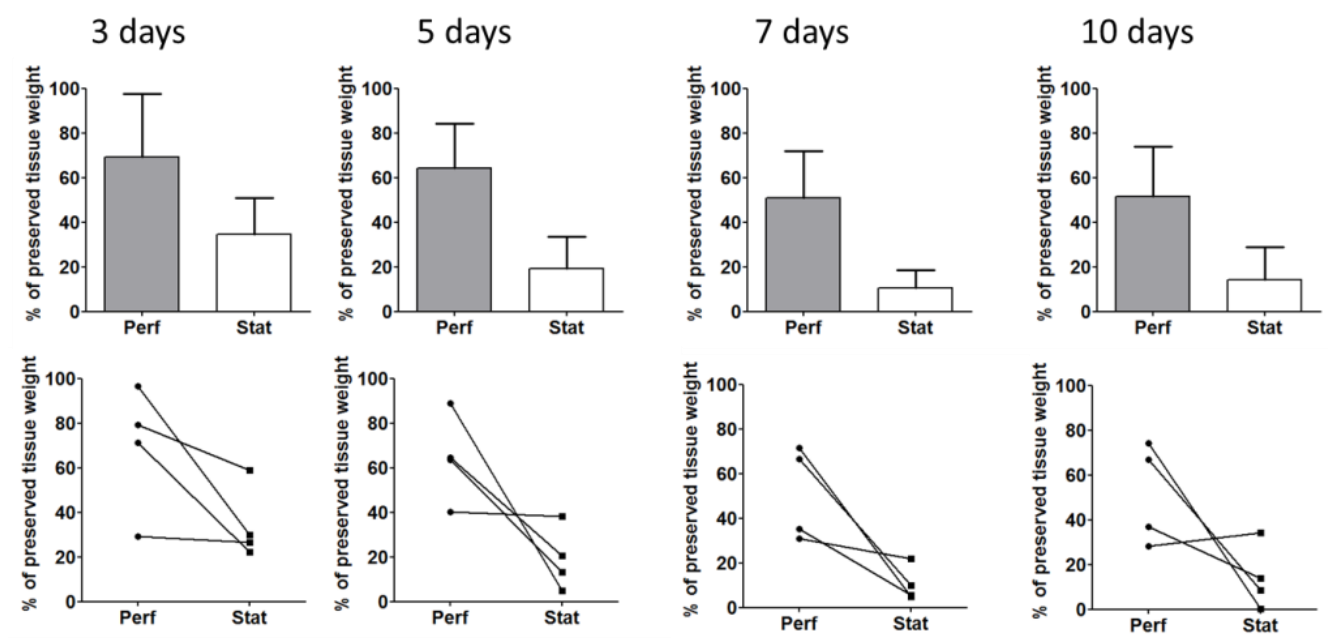

Figure VI.1 - Time point evaluation, tissue mass. Tumor fragments derived from CRC specimens were weighted, and cultured in perfused bioreactors (Perf) or under static conditions (Stat), as described in Figure III.1. After respectively 3,5,7 and 10 days, fragments were collected, weighted, and embedded in OCT for histomorphological evaluation. Weight of cultured tumor chunks is shown as percentage of that recorded prior to culture (fresh). Mean $\pm S D$ ( $n=4$, upper panel) and individual values (lower panel) are shown. 
Additional experiments need to be performed in order to address the quality and quantity of the tissue recovered from the culture in bioreactor and static conditions over time, and, most importantly, to evaluate whether all cellular TME components are also maintained.

\subsubsection{Scaffold Type}

Regarding the type of scaffold, all experiments have been performed so far on collagen type I scaffolds (Avitene ${ }^{\mathrm{TM}}$ Ultrafoam ${ }^{\mathrm{TM}}$ ). However, since tumor ECM is not only composed of collagen, but also of laminin, fibronectin and tenascin, in future experiment we intend to test different scaffold types, including Matrigel-coated scaffolds, decellularized small intestinal submucosa (SIS, Cook Biotech Inc.) and engineered stromal tissues obtained by ECM deposition and subsequent decellularization by deliberate cell-apoptosis induction ${ }^{112}$.

In particular, in preliminary experiments we evaluated whether coating with Matrigel improves tumor cellular adhesion to the scaffold. Indeed, we found that CRC cells lacking collagen binding receptors, such as HCT116 cells, adhere and proliferate to higher extents to Matrigel-coated as compared to uncoated scaffolds, and this phenomenon is dependent on Matrigel concentration.

\subsubsection{Flow rate}

Effects of flow rate on CRC cultures remain to be investigated. Indeed, capitalizing on results previously obtained in bone cell culture experiments, initial experiments were performed using a flow rate of $0.3 \mathrm{ml} /$ minute. However how the flow rate can be translated into the shear stress experienced by the chunks in culture still need to be addressed. In future studies, we plan to use an approximate mathematical model to calculate the shear stress experienced by tumor chunks under different flow rates in order to understand which one better mimic the induced physiological shear stress. Moreover since the tumor stiffness has been established to play a key role in tumor progression, in parallel, the stiffness of CRC chunks prior to or after 
culture will be evaluated by atomic force microscope in order to understand if it mimics the physiological tumor stiffness.

\subsubsection{Clinical response}

In our study we did not planned to assess the ability of our system to predict patient-specific clinical response. In fact, the experiments were conducted on patient already treated by surgery thus tumor free and the tumor relapse can only be evaluated after 5 years.

We envisage validating the system's ability to predict patient-specific clinical responses in the context of follow-up studies.

\subsection{Suitability of the perfusion-based bioreactor for the assessment of the tumor response to emerging stroma-targeted therapies}

Our culture system may also prove suitable for testing therapies whose efficacy is influenced by whole TME, such as drug-loaded nanoparticles ${ }^{113}$, or for emerging stroma-targeted therapies, including immunotherapies.

\subsubsection{Testing of novel nanoparticles-mediated drug delivery}

During my $\mathrm{PhD}$ studies I started to explore the suitability of bioreactor-based cultures for testing efficacy of nanoparticles (NPs)-mediated drug delivery.

NP-mediated drug delivery is a novel promising therapeutic approach allowing achievement of high drug concentrations at the target site, while reducing toxic effects on normal tissues. A number of factors critically impact NP distribution and efficacy of drug delivery. First, the specificity of tumor targeting is critical, since receptors targeted by NPs can often be expressed, although at low levels, also on normal cells ${ }^{114}$. NP surface charge also plays a major role. Indeed the ECM is composed of collagen fibers and negatively charged glycosaminoglycans. Therefore, positively charged NPs are retained in the ECM, whereas negatively charged NPs can more easily distribute throughout the tissues ${ }^{115}$. Moreover, 
another important aspect is NP stability which within cancer tissues may be affected by enzymatic degradation.

However, in vitro models capable of recapitulating the complexity of the TME that might provide insights on physicochemical parameters influencing effectiveness of nanoparticles as drug delivery systems are still missing.

Several nanocarrier systems for cancer treatment have been developed ${ }^{116}$ and many of them are under clinical investigation ${ }^{117}$. In particular, in CRC, poly(ethylene glycol) (PEG) NPs targeting the tumor marker carcinoembryonic antigen (CEA) have been shown to successfully improve tumor localization in vitro and in vivo, in a mouse model ${ }^{118}$. Moreover, treatment of CRC cell lines with solid lipid nanoparticles allowing simultaneous delivery of two distinct anticancer compounds, has been shown to result in enhanced mortality of tumor cells ${ }^{119}$. Furthermore, poly( $\gamma$-benzyl-L-glutamate) (PBLG) - PEG NPs, loaded with 5-FU, have shown sustained drug release, prolonged drug half-life, and increased tissue appetency in CRC cell lines and CRC xenografts ${ }^{120}$. Human serum albumin (HSA) NPs conjugated with 5fluorouracil have been showed to increase drug delivery and cytotoxicity to cells from established cell lines cancer HT-29 cells $^{121}$. Moreover, glycogen nanoparticles have been recently investigated for the construction of in vivo imaging nanoagents ${ }^{122}$. Glycogen is a biocompatible polymer, which thanks to its chemical structure cannot degrade in the bloodstream and, due its high molecular weight, is not directly eliminated by kidney ${ }^{123}$. In contrast, after internalization into cells, it is rapidly degraded into D-glucose. Notably, glycogen is internalized into tumor cells, including CRC cells, at higher levels as compared to normal cells ${ }^{124}$. Therefore, the use of glycogen-based nanocarriers may be exploited for anticancer drug delivery.

During my PhD studies, I visited the Nanostructured Interfaces and Materials Science Group (NIMS) at the Chemical Engineering department of the University of Melbourne led by Professor Frank Caruso, who has pioneered the development of new generation particle 
systems with engineered properties for targeted anti-cancer drug deliver. His group has previously developed bio-functionalized polymeric nanoparticles conjugated with an antibody specific for the A33 antigen, expressed on human CRC cells ${ }^{125,126}$ and lactosylated glycogen NPs for targeting prostate cancer cells ${ }^{127}$. Moreover, the group has developed a ultrasonic cavitation system to form in a short time HSA NPs with stable size and uniform distribution 128. In the context of collaborative studies, we have started to evaluate the suitability of our bioreactor-based system for testing the efficacy of glycogen- and HSA-NP-mediated drug delivery. Our preliminary experiment showed that the HSA NPs characterized by high biocompatibility, stability, negatively charged and with $150 \mathrm{~nm}$ diameters, can be efficiently perfused in a engineered tumor tissue able to mimic the interaction between malignant and non-transformed cells of the TME (Figue VI.2).

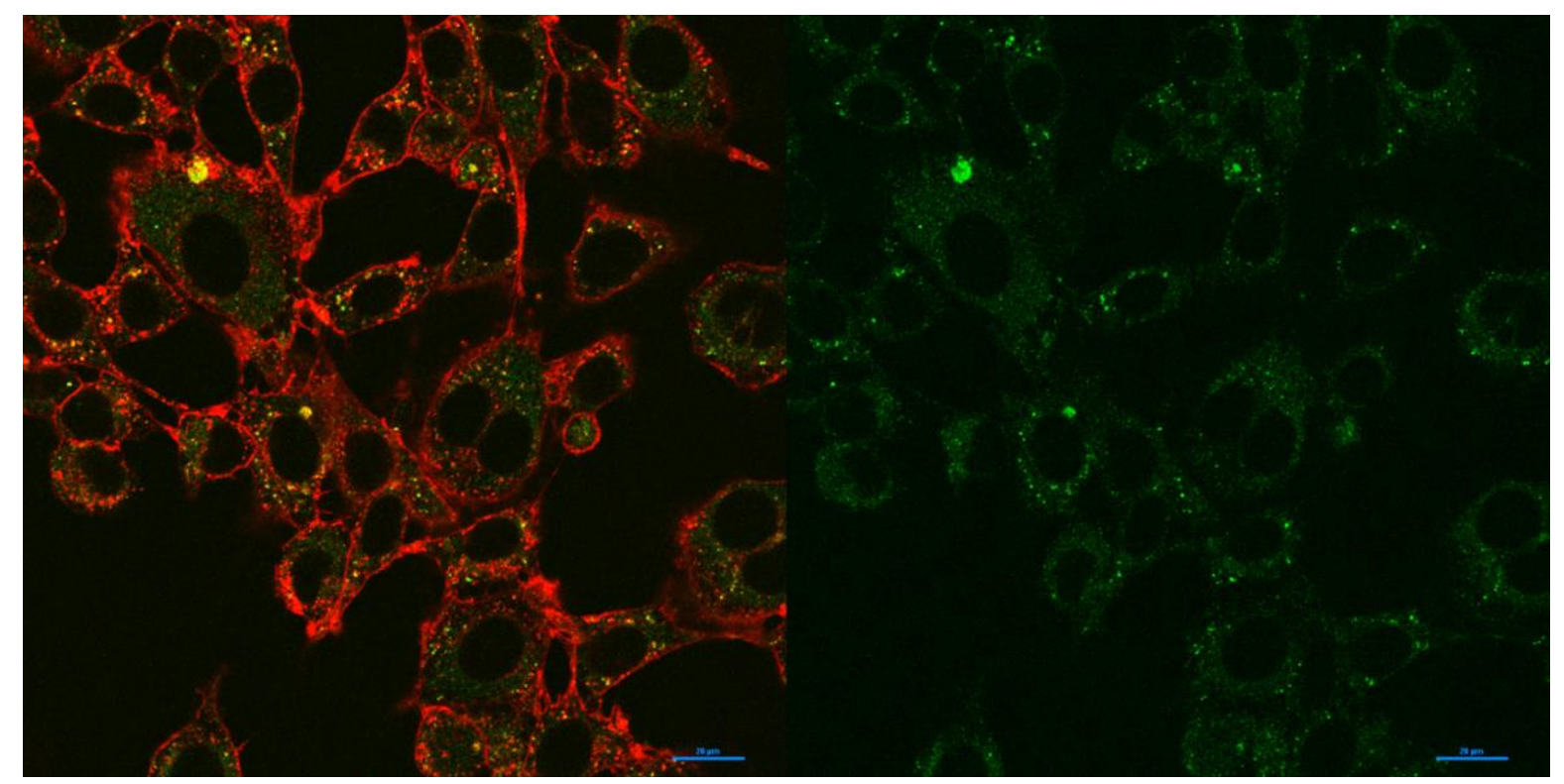

Figure VI.2 - HSA NPS can be efficiently perfused in a engineered TME. HSA NPS (150 nm) were perfused for 48 hrs in a engineered TME using the perfusion-based bioreactor. The model has been obtained by culturing HT29 CRC tumor cells and 3T3 fibroblasts for 3 weeks on a collagen type-I scaffold (Ultrafoam) in the perfusion-based bioreactor. The HSA NPS were 488-labeled (green). The scaffold was collected and embedded in OCT for histomorphological evaluation and the cell membranes were stained for wheat germ agglutinin (WGA) (red). Images were taken using a confocal at 60X magnification. 
Moreover, preliminary experiments showed that glycogen-based NPs (80-10nm) can be efficiently perfused in primary CRC tissues using the perfusion-based bioreactor (Figure VI.3).

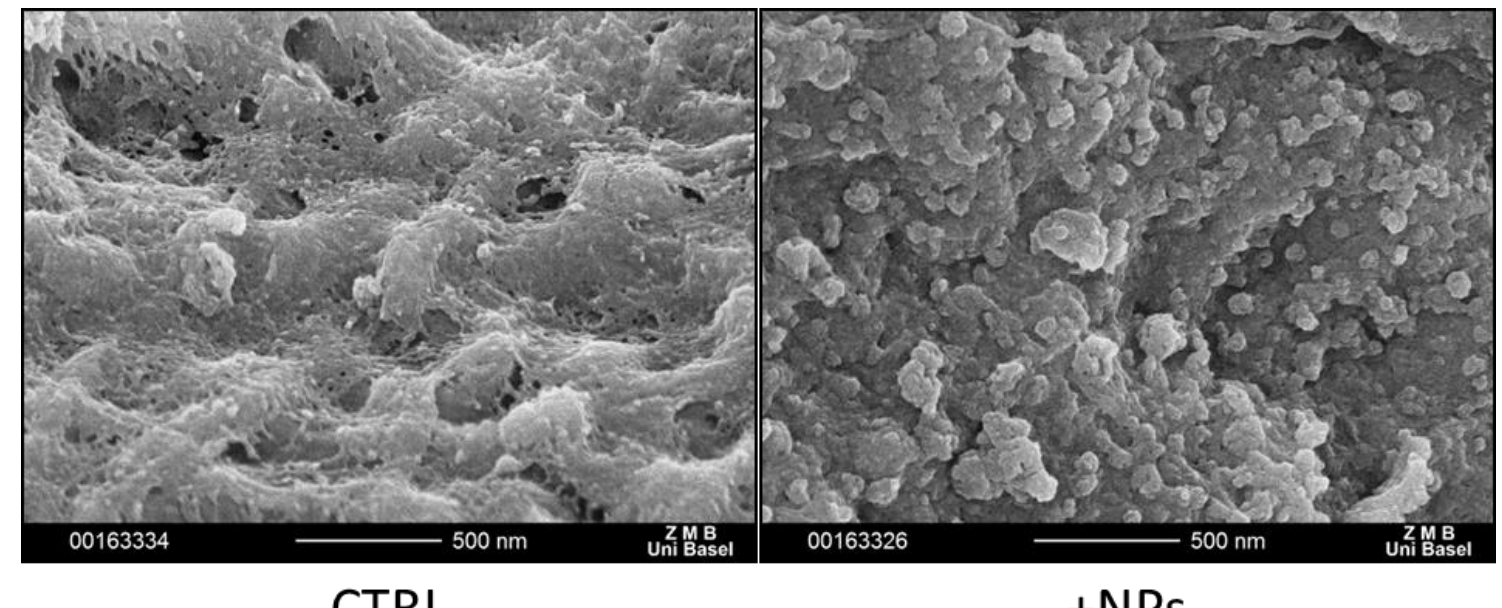

CTRL

+ NPs

Figure VI.3 - HSA NPs can be efficiently perfused in primary CRC tissues. Scanning electron microscopy (SEM) images of of primary CRC tissues cultured for 48 hrs in the perfusion-based bioreactor in the presence of glycogen-based NPs.

\subsubsection{Testing of emerging stroma-targeted therapies}

Future studies are warranted to investigate the use of perfusion-based cultures of primary CRC tissues as potential platform for emerging stroma-targeted therapies including TGF$\beta$ blockers ${ }^{129}$, BsAbs ${ }^{130}$, or immunological checkpoint inhibitors ${ }^{131}$.

The TGF- $\beta$ pathway contributes to generate a favorable microenvironment for tumor growth and metastasis throughout all the steps of carcinogenesis and so, targeting the TGF- $\beta$ pathway in cancer may be considered as a primarily microenvironment-targeted strategy. Several pharmacological approaches to block TGF- $\beta$ signaling, including monoclonal antibodies, and small molecule inhibitors, have been developed and are currently being tested in clinical trials for different cancer types, including CRC. However, there are still potential limitations and risks of TGF- $\beta$ targeted therapy including cardiotoxicity ${ }^{132}$. Thus, caution must be given as to how much therapy and when would be beneficial or how much toxicity will be induced by 
chronically administered therapy ${ }^{133}$. Therefore, systems able to predict the in vitro clinical response to TGF- $\beta$ targeted therapy are urgently needed.

BsAbs are a promising strategy to fight cancer by directly redirecting immune cells to tumor cells. In CRC, many BsAbs are now in various phases of clinical development such as the anti-CD3 +-EpCAM (MT110, BiTE, Amgen) ${ }^{134}$.

Immune checkpoint inhibitors, such as the anti-PD-1 antibody, found recent success in metastatic CRC with deficient mismatch repair system. Major challenges are ahead in order to determine how, when and which CRC patients might benefit from the use of these immune checkpoint inhibitors.

Complementary studies from our group focusing on breast cancer have recently shown that the perfusion-based bioreactor system can be efficiently used for the ex-vivo assessment of drug response to anti-programmed-death-Ligand (PD-L)-1 and anti-cytotoxic T lymphocyteassociated protein (CTLA)-4 antibodies on breast cancer primary tissue ${ }^{135}$. However, the culture conditions for tissues more difficult to maintain in vitro, such as CRC tissues, needs to be optimized.

In conclusion, novel treatments targeting non-neoplastic cells are being developed for CRC, but there are not yet systems able to predict the personal clinical response. The optimization of our perfused-based bioreactor 3D system may offer the possibility to test the efficacy of these reagents on primary tumor-associated stroma and in a patient-specific context, thus possibly paving the way towards personalized therapeutic approaches. 
BIBLIOGRAPHY 
1. Haggar FA, Boushey RP. Colorectal cancer epidemiology: incidence, mortality, survival, and risk factors. Clin Colon Rectal Surg. 2009;22(4):191-197.

2. Saunders M, Iveson T. Management of advanced colorectal cancer: state of the art. $\mathrm{Br}$ J Cancer. 2006;95(2):131-138.

3. Valle L. Genetic predisposition to colorectal cancer: where we stand and future perspectives. World J Gastroenterol. 2014;20(29):9828-9849.

4. Akinyemiju T, Wiener H, Pisu M. Cancer-related risk factors and incidence of major cancers by race, gender and region; analysis of the NIH-AARP diet and health study. BMC Cancer. 2017;17(1):597.

5. Amersi F, Agustin M, Ko CY. Colorectal cancer: epidemiology, risk factors, and health services. Clin Colon Rectal Surg. 2005;18(3):133-140.

6. Bailey $\mathrm{CE}, \mathrm{Hu} \mathrm{CY}$, You YN, et al. Increasing disparities in the age-related incidences of colon and rectal cancers in the United States, 1975-2010. JAMA Surg. 2015;150(1):17-22.

7. Ferlay J, Soerjomataram I, Dikshit R, et al. Cancer incidence and mortality worldwide: sources, methods and major patterns in GLOBOCAN 2012. Int J Cancer. 2015;136(5):E359-386.

8. Kim SE, Paik HY, Yoon H, Lee JE, Kim N, Sung MK. Sex- and gender-specific disparities in colorectal cancer risk. World J Gastroenterol. 2015;21(17):5167-5175.

9. White A, Vernon SW, Franzini L, Du XL. Racial disparities in colorectal cancer survival: to what extent are racial disparities explained by differences in treatment, tumor characteristics, or hospital characteristics? Cancer. 2010;116(19):4622-4631.

10. Kim ER, Chang DK. Colorectal cancer in inflammatory bowel disease: the risk, pathogenesis, prevention and diagnosis. World J Gastroenterol. 2014;20(29):98729881. 
11. Bedeir A, Krasinskas AM. Molecular diagnostics of colorectal cancer. Arch Pathol Lab Med. 2011;135(5):578-587.

12. Chung-Faye GA, Kerr DJ, Young LS, Searle PF. Gene therapy strategies for colon cancer. Mol Med Today. 2000;6(2):82-87.

13. Schneikert J, Behrens J. The canonical Wnt signalling pathway and its APC partner in colon cancer development. Gut. 2007;56(3):417-425.

14. Gryfe R, Swallow C, Bapat B, Redston M, Gallinger S, Couture J. Molecular biology of colorectal cancer. Curr Probl Cancer. 1997;21(5):233-300.

15. Kwong LN, Dove WF. APC and its modifiers in colon cancer. Adv Exp Med Biol. 2009;656:85-106.

16. Park JG, Kim DW, Hong CW, et al. Germ line mutations of mismatch repair genes in hereditary nonpolyposis colorectal cancer patients with small bowel cancer:

International Society for Gastrointestinal Hereditary Tumours Collaborative Study. Clin Cancer Res. 2006;12(11 Pt 1):3389-3393.

17. Kheirelseid EA, Miller N, Chang KH, et al. Mismatch repair protein expression in colorectal cancer. J Gastrointest Oncol. 2013;4(4):397-408.

18. Poynter JN, Siegmund KD, Weisenberger DJ, et al. Molecular characterization of MSI-H colorectal cancer by MLHI promoter methylation, immunohistochemistry, and mismatch repair germline mutation screening. Cancer Epidemiol Biomarkers Prev. 2008;17(11):3208-3215.

19. Ong ML, Schofield JB. Assessment of lymph node involvement in colorectal cancer. World J Gastrointest Surg. 2016;8(3):179-192.

20. Marzouk O, Schofield J. Review of histopathological and molecular prognostic features in colorectal cancer. Cancers (Basel). 2011;3(2):2767-2810.

21. Saridaki Z, Souglakos J, Georgoulias V. Prognostic and predictive significance of MSI in stages II/III colon cancer. World J Gastroenterol. 2014;20(22):6809-6814. 
22. Vilar E, Scaltriti M, Balmaña J, et al. Microsatellite instability due to hMLH1 deficiency is associated with increased cytotoxicity to irinotecan in human colorectal cancer cell lines. Br J Cancer. 2008;99(10):1607-1612.

23. Mohelnikova-Duchonova B, Melichar B, Soucek P. FOLFOX/FOLFIRI pharmacogenetics: the call for a personalized approach in colorectal cancer therapy. World J Gastroenterol. 2014;20(30):10316-10330.

24. Douillard JY, Cunningham D, Roth AD, et al. Irinotecan combined with fluorouracil compared with fluorouracil alone as first-line treatment for metastatic colorectal cancer: a multicentre randomised trial. Lancet. 2000;355(9209):1041-1047.

25. Saltz LB, Cox JV, Blanke C, et al. Irinotecan plus fluorouracil and leucovorin for metastatic colorectal cancer. Irinotecan Study Group. N Engl J Med. 2000;343(13):905-914.

26. Lee MS, Kopetz S. Current and Future Approaches to Target the Epidermal Growth Factor Receptor and Its Downstream Signaling in Metastatic Colorectal Cancer. Clin Colorectal Cancer. 2015;14(4):203-218.

27. Price TJ, Peeters M, Kim TW, et al. Panitumumab versus cetuximab in patients with chemotherapy-refractory wild-type KRAS exon 2 metastatic colorectal cancer (ASPECCT): a randomised, multicentre, open-label, non-inferiority phase 3 study. Lancet Oncol. 2014;15(6):569-579.

28. Yamaguchi T, Iwasa S, Nagashima K, et al. Comparison of Panitumumab Plus Irinotecan and Cetuximab Plus Irinotecan for KRAS Wild-type Metastatic Colorectal Cancer. Anticancer Res. 2016;36(7):3531-3536.

29. Arrington AK, Heinrich EL, Lee W, et al. Prognostic and predictive roles of KRAS mutation in colorectal cancer. Int J Mol Sci. 2012;13(10):12153-12168. 
30. Bagnasco L, Piras D, Parodi S, et al. Role of angiogenesis inhibitors in colorectal cancer: sensitive and insensitive tumors. Curr Cancer Drug Targets. 2012;12(4):303315.

31. Giantonio BJ, Catalano PJ, Meropol NJ, et al. Bevacizumab in combination with oxaliplatin, fluorouracil, and leucovorin (FOLFOX4) for previously treated metastatic colorectal cancer: results from the Eastern Cooperative Oncology Group Study E3200. J Clin Oncol. 2007;25(12):1539-1544.

32. Matos I, Elez E, Capdevila J, Tabernero J. Emerging tyrosine kinase inhibitors for the treatment of metastatic colorectal cancer. Expert Opin Emerg Drugs. 2016;21(3):267282.

33. Kircher SM, Nimeiri HS, Benson AB. Targeting Angiogenesis in Colorectal Cancer: Tyrosine Kinase Inhibitors. Cancer J. 2016;22(3):182-189.

34. Cui Y, Guo G. Immunomodulatory Function of the Tumor Suppressor p53 in Host Immune Response and the Tumor Microenvironment. Int J Mol Sci. 2016;17(11).

35. Peddareddigari VG, Wang D, Dubois RN. The tumor microenvironment in colorectal carcinogenesis. Cancer Microenviron. 2010;3(1):149-166.

36. Wei B, Zhou X, Liang C, et al. Human colorectal cancer progression correlates with LOX-induced ECM stiffening. Int J Biol Sci. 2017;13(11):1450-1457.

37. Shiga K, Hara M, Nagasaki T, Sato T, Takahashi H, Takeyama H. Cancer-Associated Fibroblasts: Their Characteristics and Their Roles in Tumor Growth. Cancers (Basel). 2015;7(4):2443-2458.

38. Owusu BY, Vaid M, Kaler P, Klampfer L. Prognostic and Predictive Significance of Stromal Fibroblasts and Macrophages in Colon Cancer. Biomark Cancer. 2015;7(Suppl 1):29-37. 
39. Tommelein J, Verset L, Boterberg T, Demetter P, Bracke M, De Wever O. Cancerassociated fibroblasts connect metastasis-promoting communication in colorectal cancer. Front Oncol. 2015;5:63.

40. Nagasaki T, Hara M, Nakanishi H, Takahashi H, Sato M, Takeyama H. Interleukin-6 released by colon cancer-associated fibroblasts is critical for tumour angiogenesis: anti-interleukin-6 receptor antibody suppressed angiogenesis and inhibited tumourstroma interaction. Br J Cancer. 2014;110(2):469-478.

41. Lahn M, Kloeker S, Berry BS. TGF-beta inhibitors for the treatment of cancer. Expert Opin Investig Drugs. 2005;14(6):629-643.

42. Liao Z, Tan ZW, Zhu P, Tan NS. Cancer-associated fibroblasts in tumor microenvironment - Accomplices in tumor malignancy. Cell Immunol. 2018.

43. Herrera M, Islam AB, Herrera A, et al. Functional heterogeneity of cancer-associated fibroblasts from human colon tumors shows specific prognostic gene expression signature. Clin Cancer Res. 2013;19(21):5914-5926.

44. Wikberg ML, Edin S, Lundberg IV, et al. High intratumoral expression of fibroblast activation protein (FAP) in colon cancer is associated with poorer patient prognosis. Tumour Biol. 2013;34(2):1013-1020.

45. Henry LR, Lee HO, Lee JS, et al. Clinical implications of fibroblast activation protein in patients with colon cancer. Clin Cancer Res. 2007;13(6):1736-1741.

46. Calon A, Lonardo E, Berenguer-Llergo A, et al. Stromal gene expression defines poor-prognosis subtypes in colorectal cancer. Nat Genet. 2015;47(4):320-329.

47. Isella $\mathrm{C}$, Terrasi A, Bellomo SE, et al. Stromal contribution to the colorectal cancer transcriptome. Nat Genet. 2015;47(4):312-319.

48. Fridman WH, Zitvogel L, Sautès-Fridman C, Kroemer G. The immune contexture in cancer prognosis and treatment. Nat Rev Clin Oncol. 2017;14(12):717-734. 
49. Ascierto PA, Kalos M, Schaer DA, Callahan MK, Wolchok JD. Biomarkers for immunostimulatory monoclonal antibodies in combination strategies for melanoma and other tumor types. Clin Cancer Res. 2013;19(5):1009-1020.

50. Ropponen KM, Eskelinen MJ, Lipponen PK, Alhava E, Kosma VM. Prognostic value of tumour-infiltrating lymphocytes (TILs) in colorectal cancer. J Pathol. 1997;182(3):318-324.

51. Galon J, Mlecnik B, Bindea G, et al. Towards the introduction of the 'Immunoscore' in the classification of malignant tumours. J Pathol. 2014;232(2):199-209.

52. Shibutani M, Maeda K, Nagahara H, et al. Tumor-infiltrating Lymphocytes Predict the Chemotherapeutic Outcomes in Patients with Stage IV Colorectal Cancer. In Vivo. 2018;32(1):151-158

53. Norton SE, Ward-Hartstonge KA, Taylor ES, Kemp RA. Immune cell interplay in colorectal cancer prognosis. World J Gastrointest Oncol. 2015;7(10):221-232.

54. Deschoolmeester V, Baay M, Lardon F, Pauwels P, Peeters M. Immune Cells in Colorectal Cancer: Prognostic Relevance and Role of MSI. Cancer Microenviron. 2011;4(3):377-392.

55. Governa V, Trella E, Mele V, et al. The Interplay Between Neutrophils and CD8. Clin Cancer Res. 2017;23(14):3847-3858.

56. Hirt C, Eppenberger-Castori S, Sconocchia G, et al. Colorectal carcinoma infiltration by myeloperoxidase-expressing neutrophil granulocytes is associated with favorable prognosis. Oncoimmunology. 2013;2(10):e25990.

57. Muz B, de la Puente P, Azab F, Azab AK. The role of hypoxia in cancer progression, angiogenesis, metastasis, and resistance to therapy. Hypoxia (Auckl). 2015;3:83-92.

58. Trédan O, Galmarini CM, Patel K, Tannock IF. Drug resistance and the solid tumor microenvironment. J Natl Cancer Inst. 2007;99(19):1441-1454. 
59. Sounni NE, Noel A. Targeting the tumor microenvironment for cancer therapy. Clin Chem. 2013;59(1):85-93.

60. Yingling JM, Blanchard KL, Sawyer JS. Development of TGF-beta signalling inhibitors for cancer therapy. Nat Rev Drug Discov. 2004;3(12):1011-1022.

61. Neuzillet C, Tijeras-Raballand A, Cohen R, et al. Targeting the TGF $\beta$ pathway for cancer therapy. Pharmacol Ther. 2015;147:22-31.

62. Kaminska B, Wesolowska A, Danilkiewicz M. TGF beta signalling and its role in tumour pathogenesis. Acta Biochim Pol. 2005;52(2):329-337.

63. Brünker P, Wartha K, Friess T, et al. RG7386, a Novel Tetravalent FAP-DR5 Antibody, Effectively Triggers FAP-Dependent, Avidity-Driven DR5 Hyperclustering and Tumor Cell Apoptosis. Mol Cancer Ther. 2016;15(5):946-957.

64. Lynch D, Murphy A. The emerging role of immunotherapy in colorectal cancer. Ann Trans1 Med. 2016;4(16):305.

65. Alsaab HO, Sau S, Alzhrani R, et al. PD-1 and PD-L1 Checkpoint Signaling Inhibition for Cancer Immunotherapy: Mechanism, Combinations, and Clinical Outcome. Front Pharmacol. 2017;8:561.

66. Sun X, Suo J, Yan J. Immunotherapy in human colorectal cancer: Challenges and prospective. World J Gastroenterol. 2016;22(28):6362-6372.

67. Vranic S. Microsatellite instability status predicts response to anti-PD-1/PD-L1 therapy regardless the histotype: A comment on recent advances. Bosn J Basic Med Sci. $2017 ; 17(3): 274-275$.

68. Bacac M, Fauti T, Sam J, et al. A Novel Carcinoembryonic Antigen T-Cell Bispecific Antibody (CEA TCB) for the Treatment of Solid Tumors. Clin Cancer Res. 2016;22(13):3286-3297.

69. Hutchinson L, Kirk R. High drug attrition rates--where are we going wrong? Nat Rev Clin Oncol. 2011;8(4):189-190. 
70. Edmondson R, Broglie JJ, Adcock AF, Yang L. Three-dimensional cell culture systems and their applications in drug discovery and cell-based biosensors. Assay Drug Dev Technol. 2014;12(4):207-218.

71. Hoarau-Véchot J, Rafii A, Touboul C, Pasquier J. Halfway between $2 \mathrm{D}$ and Animal Models: Are 3D Cultures the Ideal Tool to Study Cancer-Microenvironment Interactions? Int J Mol Sci. 2018;19(1).

72. Luca AC, Mersch S, Deenen R, et al. Impact of the $3 \mathrm{D}$ microenvironment on phenotype, gene expression, and EGFR inhibition of colorectal cancer cell lines. PLoS One. 2013;8(3):e59689.

73. Ohta Y, Sato T. Intestinal tumor in a dish. Front Med (Lausanne). 2014;1:14.

74. Noben M, Vanhove W, Arnauts K, et al. Human intestinal epithelium in a dish: Current models for research into gastrointestinal pathophysiology. United European Gastroenterol J. 2017;5(8):1073-1081.

75. Kim JB, Stein R, O'Hare MJ. Three-dimensional in vitro tissue culture models of breast cancer-- a review. Breast Cancer Res Treat. 2004;85(3):281-291.

76. Piccoli M, D'Angelo E, Crotti S, et al. Decellularized colorectal cancer matrix as bioactive microenvironment for in vitro 3D cancer research. J Cell Physiol. 2018;233(8):5937-5948.

77. Nietzer S, Baur F, Sieber S, et al. Mimicking Metastases Including Tumor Stroma: A New Technique to Generate a Three-Dimensional Colorectal Cancer Model Based on a Biological Decellularized Intestinal Scaffold. Tissue Eng Part C Methods. 2016;22(7):621-635.

78. Lin RZ, Chang HY. Recent advances in three-dimensional multicellular spheroid culture for biomedical research. Biotechnol J. 2008;3(9-10):1172-1184. 
79. Karlsson H, Fryknäs M, Larsson R, Nygren P. Loss of cancer drug activity in colon cancer HCT-116 cells during spheroid formation in a new 3-D spheroid cell culture system. Exp Cell Res. 2012;318(13):1577-1585.

80. Lu H, Stenzel MH. Multicellular Tumor Spheroids (MCTS) as a 3D In Vitro Evaluation Tool of Nanoparticles. Small. 2018;14(13):e1702858.

81. Ricci-Vitiani L, Lombardi DG, Pilozzi E, et al. Effect of HIF-1 modulation on the response of two- and three-dimensional cultures of human colon cancer cells to 5fluorouracil.

82. Kondo J, Endo H, Okuyama H, et al. Retaining cell-cell contact enables preparation and culture of spheroids composed of pure primary cancer cells from colorectal cancer. Proc Natl Acad Sci U S A. 2011;108(15):6235-6240.

83. Sato T, Vries RG, Snippert HJ, et al. Single Lgr5 stem cells build crypt-villus structures in vitro without a mesenchymal niche. Nature. 2009;459(7244):262-265.

84. Young M, Reed KR. Organoids as a Model for Colorectal Cancer. Curr Colorectal Cancer Rep. 2016;12(5):281-287.

85. Grabinger T, Luks L, Kostadinova F, et al. Ex vivo culture of intestinal crypt organoids as a model system for assessing cell death induction in intestinal epithelial cells and enteropathy. Cell Death Dis. 2014;5:e1228.

86. Friedrich J, Seidel C, Ebner R, Kunz-Schughart LA. Spheroid-based drug screen: considerations and practical approach. Nat Protoc. 2009;4(3):309-324.

87. Majumder B, Baraneedharan U, Thiyagarajan S, et al. Predicting clinical response to anticancer drugs using an ex vivo platform that captures tumour heterogeneity. Nat Commun. 2015;6:6169.

88. Sobrino A, Phan DT, Datta R, et al. 3D microtumors in vitro supported by perfused vascular networks. Sci Rep. 2016;6:31589. 
89. Moya M, Tran D, George SC. An integrated in vitro model of perfused tumor and cardiac tissue. Stem Cell Res Ther. 2013;4 Suppl 1:S15.

90. Wan X, Li Z, Ye H, Cui Z. Three-dimensional perfused tumour spheroid model for anti-cancer drug screening. Biotechnol Lett. 2016;38(8):1389-1395.

91. Hirt C, Papadimitropoulos A, Muraro MG, et al. Bioreactor-engineered cancer tissuelike structures mimic phenotypes, gene expression profiles and drug resistance patterns observed "in vivo". Biomaterials. 2015;62:138-146.

92. Brown KM, Xue A, Mittal A, Samra JS, Smith R, Hugh TJ. Patient-derived xenograft models of colorectal cancer in pre-clinical research: a systematic review. Oncotarget. 2016;7(40):66212-66225.

93. Rosfjord E, Lucas J, Li G, Gerber HP. Advances in patient-derived tumor xenografts: from target identification to predicting clinical response rates in oncology. Biochem Pharmacol. 2014;91(2):135-143.

94. Chao C, Widen SG, Wood TG, et al. Patient-derived Xenografts from Colorectal Carcinoma: A Temporal and Hierarchical Study of Murine Stromal Cell Replacement. Anticancer Res. 2017;37(7):3405-3412.

95. Fatehullah A, Tan SH, Barker N. Organoids as an in vitro model of human development and disease. Nat Cell Biol. 2016;18(3):246-254.

96. Papadimitropoulos A, Scherberich A, Güven S, et al. A 3D in vitro bone organ model using human progenitor cells. Eur Cell Mater. 2011;21:445-458; discussion 458.

97. Cerino G, Gaudiello E, Grussenmeyer T, et al. Three dimensional multi-cellular muscle-like tissue engineering in perfusion-based bioreactors. Biotechnol Bioeng. 2016;113(1):226-236.

98. Braccini A, Wendt D, Jaquiery C, et al. Three-dimensional perfusion culture of human bone marrow cells and generation of osteoinductive grafts. Stem Cells. 2005;23(8):1066-1072. 
99. Cioffi M, Kuffer J, Strobel S, Dubini G, Martin I, Wendt D. Computational evaluation of oxygen and shear stress distributions in 3D perfusion culture systems: macro-scale and micro-structured models. J Biomech. 2008;41(14):2918-2925.

100. Santoro M, Lamhamedi-Cherradi SE, Menegaz BA, Ludwig JA, Mikos AG. Flow perfusion effects on three-dimensional culture and drug sensitivity of Ewing sarcoma. Proc Natl Acad Sci U S A. 2015;112(33):10304-10309.

101. Radisic M, Deen W, Langer R, Vunjak-Novakovic G. Mathematical model of oxygen distribution in engineered cardiac tissue with parallel channel array perfused with culture medium containing oxygen carriers. Am J Physiol Heart Circ Physiol. 2005;288(3):H1278-1289.

102. Carpenter AE, Jones TR, Lamprecht MR, et al. CellProfiler: image analysis software for identifying and quantifying cell phenotypes. Genome Biol. 2006;7(10):R100.

103. Kyurkchiev D, Bochev I, Ivanova-Todorova E, et al. Secretion of immunoregulatory cytokines by mesenchymal stem cells. World J Stem Cells. 2014;6(5):552-570.

104. Gubin MM, Zhang X, Schuster H, et al. Checkpoint blockade cancer immunotherapy targets tumour-specific mutant antigens. Nature. 2014;515(7528):577-581.

105. Schumacher TN, Schreiber RD. Neoantigens in cancer immunotherapy. Science. 2015;348(6230):69-74.

106. Yamada KM, Cukierman E. Modeling tissue morphogenesis and cancer in 3D. Cell. 2007;130(4):601-610.

107. Ricci-Vitiani L, Lombardi DG, Pilozzi E, et al. Identification and expansion of human colon-cancer-initiating cells. Nature. 2007;445(7123):111-115.

108. Todaro M, Alea MP, Di Stefano AB, et al. Colon cancer stem cells dictate tumor growth and resist cell death by production of interleukin-4. Cell Stem Cell. 2007;1(4):389-402. 
109. Sato T, Stange DE, Ferrante M, et al. Long-term expansion of epithelial organoids from human colon, adenoma, adenocarcinoma, and Barrett's epithelium. Gastroenterology. 2011;141(5):1762-1772.

110. Tashiro T, Okuyama $\mathrm{H}$, Endo $\mathrm{H}$, et al. In vivo and ex vivo cetuximab sensitivity assay using three-dimensional primary culture system to stratify KRAS mutant colorectal cancer. PLoS One. 2017;12(3):e0174151.

111. Estes BT, Gimble JM, Guilak F. Mechanical signals as regulators of stem cell fate. Curr Top Dev Biol. 2004;60:91-126.

112. Bourgine PE, Pippenger BE, Todorov A, Tchang L, Martin I. Tissue decellularization by activation of programmed cell death. Biomaterials. 2013;34(26):6099-6108.

113. Rama AR, Jimenez-Lopez J, Cabeza L, et al. Last Advances in Nanocarriers-Based Drug Delivery Systems for Colorectal Cancer. Curr Drug Deliv. 2016;13(6):830-838.

114. Bartoş A, Bartoş D, Szabo B, et al. Recent achievements in colorectal cancer diagnostic and therapy by the use of nanoparticles. Drug Metab Rev. 2016;48(1):2746.

115. Kapadia CH, Perry JL, Tian S, Luft JC, DeSimone JM. Nanoparticulate immunotherapy for cancer. J Control Release. 2015;219:167-180.

116. Prados J, Melguizo C, Ortiz R, et al. Colon cancer therapy: recent developments in nanomedicine to improve the efficacy of conventional chemotherapeutic drugs. Anticancer Agents Med Chem. 2013;13(8):1204-1216.

117. Dong Z, Cui MY, Peng Z, et al. Nanoparticles for Colorectal Cancer Targeted Drug Delivery and MR Imaging: Current Situation and Perspectives. Curr Cancer Drug Targets. 2016;16(6):536-550.

118. Tiernan JP, Ingram N, Marston G, et al. CEA-targeted nanoparticles allow specific in vivo fluorescent imaging of colorectal cancer models. Nanomedicine (Lond). 2015;10(8):1223-1231. 
119. Tummala S, Satish Kumar MN, Prakash A. Formulation and characterization of 5Fluorouracil enteric coated nanoparticles for sustained and localized release in treating colorectal cancer. Saudi Pharm J. 2015;23(3):308-314.

120. Li S, Wang A, Jiang W, Guan Z. Pharmacokinetic characteristics and anticancer effects of 5-fluorouracil loaded nanoparticles. BMC Cancer. 2008;8:103.

121. Sharma A, Kaur A, Jain UK, Chandra R, Madan J. Stealth recombinant human serum albumin nanoparticles conjugating 5-fluorouracil augmented drug delivery and cytotoxicity in human colon cancer, HT-29 cells. Colloids Surf B Biointerfaces. 2017;155:200-208.

122. Aasen SN, Pospisilova A, Eichler TW, et al. A Novel Nanoprobe for Multimodal Imaging Is Effectively Incorporated into Human Melanoma Metastatic Cell Lines. Int J Mol Sci. 2015;16(9):21658-21680.

123. Filippov SK, Sedlacek O, Bogomolova A, et al. Glycogen as a biodegradable construction nanomaterial for in vivo use. Macromol Biosci. 2012;12(12):1731-1738.

124. Takahashi S, Satomi A, Yano K, et al. Estimation of glycogen levels in human colorectal cancer tissue: relationship with cell cycle and tumor outgrowth. J Gastroenterol. 1999;34(4):474-480.

125. Cortez C, Tomaskovic-Crook E, Johnston AP, et al. Influence of size, surface, cell line, and kinetic properties on the specific binding of A33 antigen-targeted multilayered particles and capsules to colorectal cancer cells. ACS Nano. 2007;1(2):93-102.

126. Johnston AP, Kamphuis MM, Such GK, et al. Targeting cancer cells: controlling the binding and internalization of antibody-functionalized capsules. ACS Nano. 2012;6(8):6667-6674. 
127. Besford QA, Wojnilowicz M, Suma T, Bertleff-Zieschang N, Caruso F, Cavalieri F. Lactosylated Glycogen Nanoparticles for Targeting Prostate Cancer Cells. ACS Appl Mater Interfaces. 2017;9(20):16869-16879.

128. Francesca C, Enrico C, Eleonora N, Nicola R, Muthupandian A. Sono-assembly of nanostructures via tyrosine-tyrosine coupling reactions at the interface of acoustic cavitation bubbles. Materials Horizons. 2016;3(6):563-567.

129. de Gramont A, Faivre S, Raymond E. Novel TGF-beta inhibitors ready for prime time in onco-immunology. Oncoimmunology. 2017;6(1):e1257453.

130. Lehmann S, Perera R, Grimm HP, et al. In Vivo Fluorescence Imaging of the Activity of CEA TCB, a Novel T-Cell Bispecific Antibody, Reveals Highly Specific Tumor Targeting and Fast Induction of T-Cell-Mediated Tumor Killing. Clin Cancer Res. 2016;22(17):4417-4427.

131. Nebot-Bral L, Brandao D, Verlingue L, et al. Hypermutated tumours in the era of immunotherapy: The paradigm of personalised medicine. Eur J Cancer. 2017;84:290303.

132. Kovacs RJ, Maldonado G, Azaro A, et al. Cardiac Safety of TGF- $\beta$ Receptor I Kinase Inhibitor LY2157299 Monohydrate in Cancer Patients in a First-in-Human Dose Study. Cardiovasc Toxicol. 2015;15(4):309-323.

133. Kubiczkova L, Sedlarikova L, Hajek R, Sevcikova S. TGF- $\beta$ - an excellent servant but a bad master. J Trans1 Med. 2012;10:183.

134. Zhang X, Yang Y, Fan D, Xiong D. The development of bispecific antibodies and their applications in tumor immune escape. Exp Hematol Oncol. 2017;6:12.

135. Muraro MG, Muenst S, Mele V, et al. assessment of drug response on breast cancer primary tissue with preserved microenvironments. Oncoimmunology. 2017;6(7):e1331798. 


\section{ACKNOWLEDGEMENTS}


"I know that I know nothing"; I would like to start my acknowledgments with this Socrate's famous philosophical quote. I understand this is not a common way to start thesis acknowledgments. However, this feeling accompanied me over these past four years and drove my thirst for knowledge.

I developed this thought thanks to all the people who crossed my path during my $\mathrm{PhD}$ studies. In particular, I would like to thank my PhD advisors, Professors Ivan Martin and Giandomenica Iezzi, for being such inspiring mentors and for supporting me during these past four years. I would like to thank you for encouraging me in my research and for allowing me to grow as a research scientist. I am honored you gave me the opportunity to pursue this interesting project and the freedom to bring my own ideas to it. Thank you for the opportunity to visit the University of Melbourne for six months during my $\mathrm{PhD}$ studies. This experience definitely contributed to my personal and professional growth. A special thanks goes to Professor Iezzi for the constant encouragement. Her support was crucial during my $\mathrm{PhD}$ studies. She spent endless hours proofreading my research papers, grants, projects and this dissertation and gave me excellent suggestions.

I would like to express my sincere gratitude to Professor Luigi Terracciano for the incredible effort with my $\mathrm{PhD}$ project. Without his continuous guidance and insight, I could not have completed this work. He has always been extremely supportive, always available despite his busy schedule to help me. Thank you for sharing not only your knowledge, but most importantly, the passion for your job with me.

I am truly grateful to Professor Giulio Spagnoli for his detailed and constructive comments which were vital to the development of my $\mathrm{PhD}$ project.

I wish to thank Professor Gerhard Christofori, member of my $\mathrm{PhD}$ committee, for offering me his time, support and encouragement and for the brilliant comments and suggestions that definitely improved my research. 
My sincere gratitude to Dr. Valentina Mele for her endless patience, teachings and insights. She was always present and ready to help me and give me valuable advice. She gave me the basis necessary to work independently and she supervised my results with meticulous attention.

I would like to thank Dr. Manuele Muraro who truly pushed me out from my comfort zone and encouraged me to learn more, to do more and to always do better. Thank you so much for all the time you spent sharing your insights and knowledge with me.

I would like to express my deepest gratitude to our collaborator Professor Marija Plodinec for supporting my research with enthusiasm and for the valuable advice that truly progressed the project.

My sincere thanks to Professor Susan Treves for kindly willing to chair my $\mathrm{PhD}$ dissertation.

Also, I would really like to thank Dr. Michael Abanto for all the valuable insight and effort with image analysis.

Moreover, I am grateful to Professor Frank Caruso and Dr. Francesca Cavalieri for the opportunity to pursue part of my $\mathrm{PhD}$ traning in their laboratories and for their valuable suggestions, ideas and the important contribution to my research.

Finally, I would like to thank my colleagues who helped me to quickly learn all the technical skills necessary to advance in my $\mathrm{PhD}$ project. In particular, a special thanks to Dr. Valeria Governa for all the insight into immunology. She has always been kindly available for clarifications, advice and help with experiments. A special thanks to Laura Power for proofreading grants and the present thesis dissertation.

My sincere gratitude to Dr. Britta Luescher for being my mentor during the past 4 years. She encouraged me to follow my dreams and helped me to define the step necessary to reach my goals. 
Thank you for being such a great role model. It would be impossible to count all the ways that you've helped me in my career. Thank you so much for all that you've done, I will forever be grateful for your guidance and kindness.

Also, I would really like to thank Dr. Federico Tortelli; Thank you for seeing my potential when I arrived in Switzerland 5 years ago. Thank you for all your valuable teaching, contribute not only to my professional but also to my personal growth.

Last but not least, I want to thank my family, in particular my father and mother who gave me endless support. Thank you for believing in me even when I was the first to doubt. You gave me the strength to take every chance that came into my life with joy and courage. A special thanks to my dad. Thank you so much for still being here to hold my hand and to celebrate this important accomplishment. Also, I want to express my sincere gratitude to my grandmother, my tireless confidant and guide. I want to sincerely thank my best friends Gloria, Dora, Claudia and my cousin Enrica for always being by my side. During the past five years they represented my anchor and they are the only thing that has never changed in my life. Finally, I want to express my deepest gratitude to Giulia, you have been not only a very good friend but my family here in Basel. Your support, encouragement and advice have been extremely important to me. I found a sister far from home! 
APPENDIX 


\title{
Maintenance of Primary Human Colorectal Cancer Microenvironment Using a Perfusion Bioreactor-Based 3D Culture System
}

\author{
Celeste Manfredonia, Manuele G. Muraro, Christian Hirt, Valentina Mele, Valeria Governa, \\ Adam Papadimitropoulos, Silvio Däster, Savas D. Soysal, Raoul A. Droeser, Robert Mechera, \\ Daniel Oertli, Raffaele Rosso, Martin Bolli, Andreas Zettl, Luigi M. Terracciano, \\ Giulio C. Spagnoli, Ivan Martin,** and Giandomenica Iezzi**
}

Colorectal cancer (CRC) is a leading cause of cancer-related death. Conventional chemotherapeutic regimens have limited success rates, and a major challenge for the development of novel therapies is the lack of adequate in vitro models. Nonmalignant mesenchymal and immune cells of the tumor microenvironment (TME) are known to critically affect CRC progression and drug responsiveness. However, tumor drug sensitivity is still evaluated on systems, such as cell monolayers, spheroids, or tumor xenografts, which typically neglect the original TME. Here, it is investigated whether a bioreactorbased 3D culture system can preserve the main TME cellular components in primary CRC samples. Freshly excised CRC fragments are inserted between two collagen scaffolds in a "sandwich-like" format and cultured under static or perfused conditions up to $3 \mathrm{~d}$. Perfused cultures maintain tumor tissue architecture and densities of proliferating tumor cells to significantly higher extents than static cultures. Stromal and immune cells are also preserved and fully viable, as indicated by their responsiveness to microenvironmental stimuli. Importantly, perfusion-based cultures prove suitable for testing the sensitivity of primary tumor cells to chemotherapies currently in use for CRC. Perfusionbased culture of primary CRC specimens recapitulates TME key features and may allow assessment of tumor drug response in a patient-specific context.

\section{Introduction}

Colorectal cancer (CRC) is a leading cause of cancer-related death worldwide. ${ }^{[1]}$ Whereas surgery represents the first therapeutic option for early stage disease, in more advanced stages the treatment includes chemotherapy, generally based on the administration of 5-fluorouracil (5-FU), in association with oxaliplatin (FOLFOX) or irinotecan (FOLFIRI). However, these chemotherapy protocols are characterized by low response rates, usually not exceeding $20 \%$ of treated patients. ${ }^{[2]}$

The development of novel, more effective therapies is hampered by the lack of culture systems adequately mimicking the architectural and cellular complexity of the tumor microenvironment (TME). Nontransformed cells within the TME, including so-called tumor-associated mesenchymal stromal cells and tumorinfiltrating immune cells, have been recognized to profoundly affect response
Dr. C. Manfredonia, Dr. V. Mele, Dr. V. Governa, Prof. G. lezzi

Cancer Immunotherapy

Department of Biomedicine

University Hospital Basel

University of Basel

Basel 4031, Switzerland

E-mail: giandomenica.iezzi@eoc.ch

Dr. C. Manfredonia, Dr. M. G. Muraro, Dr. C. Hirt,

Dr. A. Papadimitropoulos, Prof. I. Martin

Tissue Engineering

Department of Biomedicine

University Hospital Basel

University of Basel

Basel 4031, Switzerland

E-mail: ivan.martin@usb.ch

The ORCID identification number(s) for the author(s) of this article can be found under https://doi.org/10.1002/adbi.201800300.

DOI: 10.1002/adbi.201800300

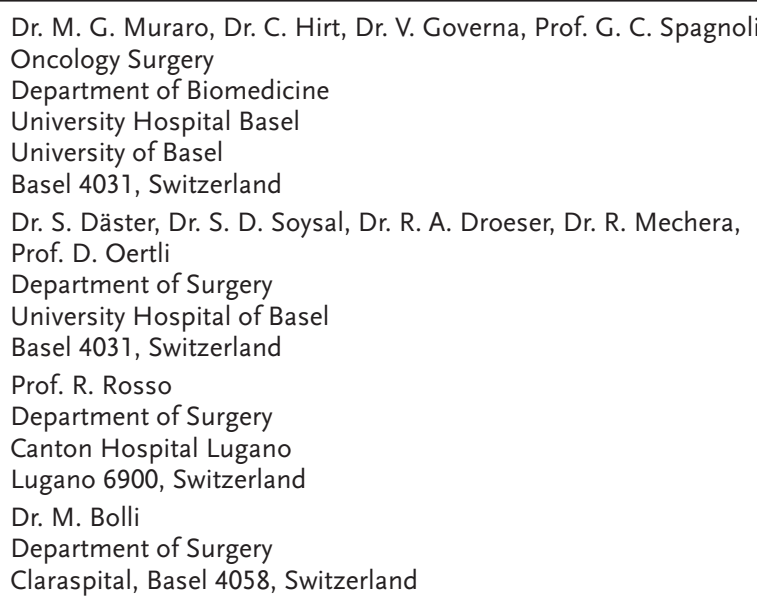


to treatment and tumor clinical outcome. ${ }^{[3-5]}$ Indeed, mesenchymal-related signatures have been found to predict poor clinical outcome ${ }^{[6,7]}$ and relapse upon therapy. ${ }^{[8]}$ Furthermore, CRC-derived stromal cells have been shown to promote chemoresistance of CRC cells to 5-FU and oxaliplatin in vitro. ${ }^{[9]}$

In contrast, CRC-infiltrating immune cells, and in particular CD8+ T lymphocytes and T-helper 1 cells, have been recognized to predict improved prognosis irrespective of tumor stage. ${ }^{[10]}$ Underlying mechanisms are still largely unclear. T lymphocytes might kill tumor cells upon recognition of tumorassociated (neo)-antigens. ${ }^{[11]}$ Furthermore, defined $\mathrm{T}$ cell subsets may be required for a full elicitation of chemotherapy effectiveness, as observed in mouse models of lung and breast cancer. $^{[12]}$ Finally, immune cells expressing Fc fragment receptors, including NK and myeloid cells, might contribute to therapeutic efficacy of growth factor receptor-specific monoclonal antibodies (mAbs) through antibody-dependent cell cytotoxicity (ADCC). ${ }^{[13]}$

Despite this evidence, drug screening is presently largely based on the use of established cell lines cultured in 2D tumor cell monolayers. More recently, multicellular tumor spheroids from cell lines ${ }^{[14]}$ or primary tumor cells ${ }^{[15,16]}$ and organoids from primary CRC tissues ${ }^{[17]}$ have been shown to mimic morphological and biochemical features of in vivo tumors and have been proposed to assess efficacy of novel compounds. ${ }^{[14,18]}$ However, these systems still lack the interaction between tumor cells and nontransformed components of cancer tissues. ${ }^{[19]}$ Improved spheroid-based cultures, also including stromal and endothelial cells, have been recently proposed, but, so far, only for culture of tumor cells derived from established cell lines. ${ }^{[20-22]}$

Alternatively, tumor xenografts, generated upon injection of cell lines in immunodeficient mice, and in particular patient derived xenograft (PDX) generated upon injection or implantation of primary tumor cells, are also used. PDXs retain to remarkable extents biological, histological, genomic, gene, and biomarker expression profiles of their tumor of origin. ${ }^{[23]}$ However, they lack human stromal and immune cells, which are rapidly replaced by murine cells. ${ }^{[24]}$ Thus, the establishment of new technologies reliably allowing the maintenance of the whole human TME is critically required.

In the last few years, perfusion-based cell cultures have demonstrated a unique ability to promote generation of tissue constructs displaying biological and structural characteristics comparable with those of primary tissues. ${ }^{[25,26]}$ Previous studies from our group have addressed the suitability of a perfused bioreactor for tumor engineering purposes. Culture of CRC cells

\footnotetext{
Dr. A. Zettl

Viollier AG

Allschwil 4123, Switzerland

Prof. L. M. Terracciano

Institute of Pathology

University Hospital Basel

Basel 4056, Switzerland

Prof. G. lezzi

Department of Surgery

Ente Ospedaliero Cantonale

and Università Svizzera Italiana

Bellinzona 6500, Switzerland
}

from established cell lines on collagen scaffold in this culture system, resulted in formation of tumor tissue-like structures displaying structural, molecular and drug sensitivity profiles similar to those of tissues generated in xenografts. ${ }^{[27]}$ More recently, this perfused bioreactor has proved helpful for midterm culture of human breast cancer tissues. ${ }^{[28]}$ However, its suitability, for tumor types more difficult to culture in vitro, such as CRC, ${ }^{[29]}$ has not been tested yet.

Capitalizing on these data, in this work we have investigated the suitability of perfusion-based culture for the maintenance of the main cellular components of the TME of human primary CRC.

\section{Results}

\subsection{Culture under Perfusion Preserves the Heterogeneity of CRC Microenvironment}

Tumor fragments from freshly resected human CRC specimens were placed between two collagen scaffolds in a sandwich-like configuration and cultured under perfusion in a previously described bioreactor ${ }^{[27,30]}$ up to $3 \mathrm{~d}$, a time point used in previous studies ${ }^{[31]}$ to assess drug responsiveness on human CRC tissues (Figure 1). Tissue valuable for subsequent analysis was recovered in 23 out of 23 samples.

As static culture controls, in initial experiments tissue samples were embedded in sandwich scaffolds and maintained in bioreactors without perfusion. However, these culture conditions resulted in complete tissue loss, precluding subsequent analysis (data not shown). Therefore, as alternative static condition, tumor fragments were seeded in conventional culture plates (Figure 1), a condition allowing the recovery of at least one tumor fragment out of three in all experiments performed $(n=17)$.

Tissue mass analysis performed before and after culture showed that perfused cultures preserved up to $75 \pm 8 \%$ of tissue weight, compared to $31 \pm 16 \%$ only in static cultures $(p=0.078$, Figure 2A). Accordingly, on histomorphological analysis, perfused tissues maintained higher tissue cellularity, as indicated by total nuclei counts, in comparison to static cultures $(68 \pm 20$ and $40 \pm 23 \%$, respectively, $p=0.02$, Figure $2 \mathrm{~B}$ and Figure S1A, Supporting Information).

Importantly, evaluation of tissue quality and composition showed that original architecture was partially maintained in CRC tissues cultured in the perfused bioreactor, whereas it was completely lost in nonperfused cultures (Figure 2C). In particular, in cultures under perfusion the epithelial component was only partially and not significantly reduced as compared to fresh tissues $(35 \pm 14$ vs $59 \pm 27 \%$, respectively, $p$-value $>0.1$ ) In contrast, tumor tissues cultured under static conditions displayed significant reductions of epithelial cell fraction $(10 \pm 13 \%, p=0.022$ vs fresh tissues, $p=0.016$ vs perfused cultures) (Figure 2D). Notably, in four out of seven samples evaluated, the epithelial fraction was completely lost (Figure S1B, Supporting Information). Numbers of mesenchymal stromal cells, as assessed upon vimentin staining, were also comparable in fresh tissues and tissues cultured under perfusion (out of five ROI: $604 \pm 376$ and $465 \pm 227$ positive cells, respectively, 


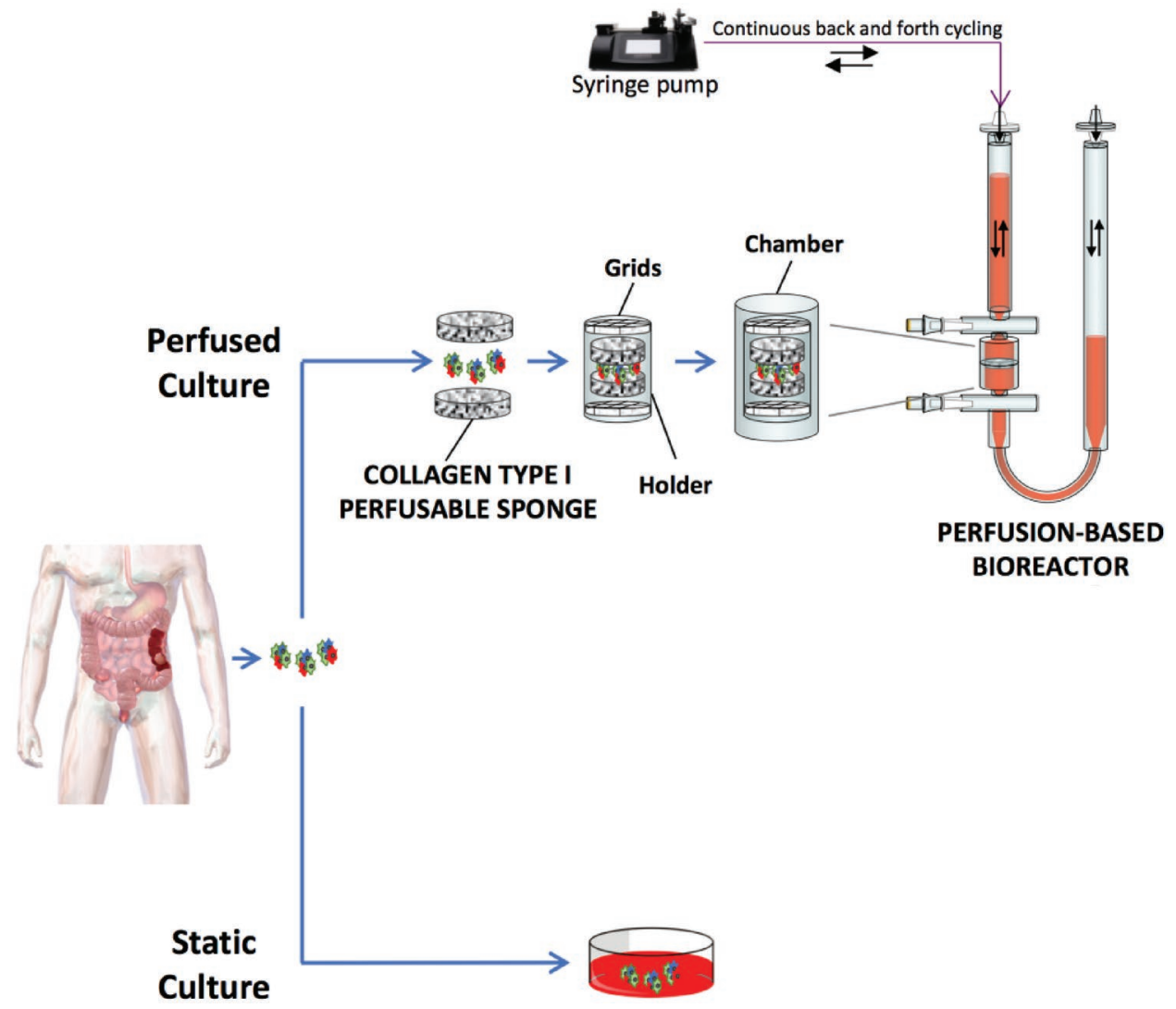

Figure 1. Schematic representation of the experimental design. Freshly resected CRC specimens were fragmented in $2 \times 2 \times 2 \mathrm{~mm}$ chunks. For perfused cultures, tumor fragments ( $n=3 /$ bioreactor) were placed between two collagen type I discs within a ring-shaped holder, restrained by two grids on the top and bottom. The holder was then inserted in the bioreactor chamber and subjected to continuous alternate perfusion. For static cultures, tumor chunks ( $n=3 /$ plate) were seeded in conventional culture plates. Perfused and static cultures were incubated at $37^{\circ} \mathrm{C}$ for $3 \mathrm{~d}$.

$p>0.3)$. However, they were significantly reduced in static cultures (128 \pm 102 positive cells), as compared to both fresh tissues and perfused cultures $(p=0.008$ and 0.016 , respectively) (Figure 2E and Figure S1B, Supporting Information). Accordingly, tumor/stroma ratios were more strongly reduced upon static than perfused cultures (Figure S1C, Supporting Information).

Tumor-infiltrating immune cells, identified as CD45+ cells, although being markedly reduced in both culture types, were still clearly detectable in perfused tissues (out of five ROI: $75 \pm 127$ cells vs $173 \pm 145$ in fresh tissues, $p>0.05$ ), whereas they were almost completely lost upon static culture $(9 \pm 9$, $p=0.008$ vs fresh tissues, $p=0.054$ vs perfused cultures, Figure $2 \mathrm{~F}$ and Figure S1B, Supporting Information). Characterization of immune cell subsets (Figure S2, Supporting Information) revealed that proportions of $\mathrm{T}$ lymphocytes $(\mathrm{CD} 3+)$ were comparable in fresh tissues and perfused cultures $(26.51 \pm 18.3$ vs $23.8 \pm 13.6 \%$ of total CD45+ cells $)$, whereas they were significantly reduced in static cultures $(2.3 \pm 3 \%, p$ vs fresh $=0.02)$. Few B lymphocytes $(C D 19+)$ were detected in fresh CRC tissues $(7.3 \pm 8.4 \%$ of CD45+ cells). Upon culture under perfusion they were markedly reduced but still detectable $(1.7 \pm 2.1 \% p=$ n.s. $)$, whereas upon static cultures they were completely lost. Finally, macrophages (CD64+) were detected in similar proportions in all culture conditions $(6.6 \pm 12.2$ in fresh tissues, $7.3 \pm 6.6$ in perfused culture, $5.6 \pm 6.5$ in static cultures).

Endothelial cells, as assessed by CD31 expression, were preserved in both culture conditions in amounts comparable to those found in fresh tissues (Figure S3, Supporting Information). Finally, when we evaluated distribution of collagen, one of major components of CRC extracellular matrix, ${ }^{[31]}$ we did not observe major differences between fresh and cultured tissues (Figure S4, Supporting Information).

These results cumulatively indicated that short-term culture of primary tumor fragments under perfusion maintains heterogeneity of CRC cellular components to significantly higher extents, as compared to static cultures.

\subsection{Perfused Cultures Better Preserved Viability and Functionality of All CRC Cellular Components}

To further assess cell viability within cultured tissues, we evaluated proportions of proliferating or apoptotic cells, upon Ki67 or cC3 staining, respectively (Figure 3 and Figure S5, Supporting Information). In perfused cultures, proliferation and apoptotic rates were comparable to those observed in fresh tissues (Ki67+ cells: $5 \pm 5 \%$ vs $6 \pm 4 \%$; cC $3+$ cells: $7 \pm 7 \%$ vs $5 \pm 4 \%$, respectively, Figure 3B,C and Figure S5, Supporting Information). 

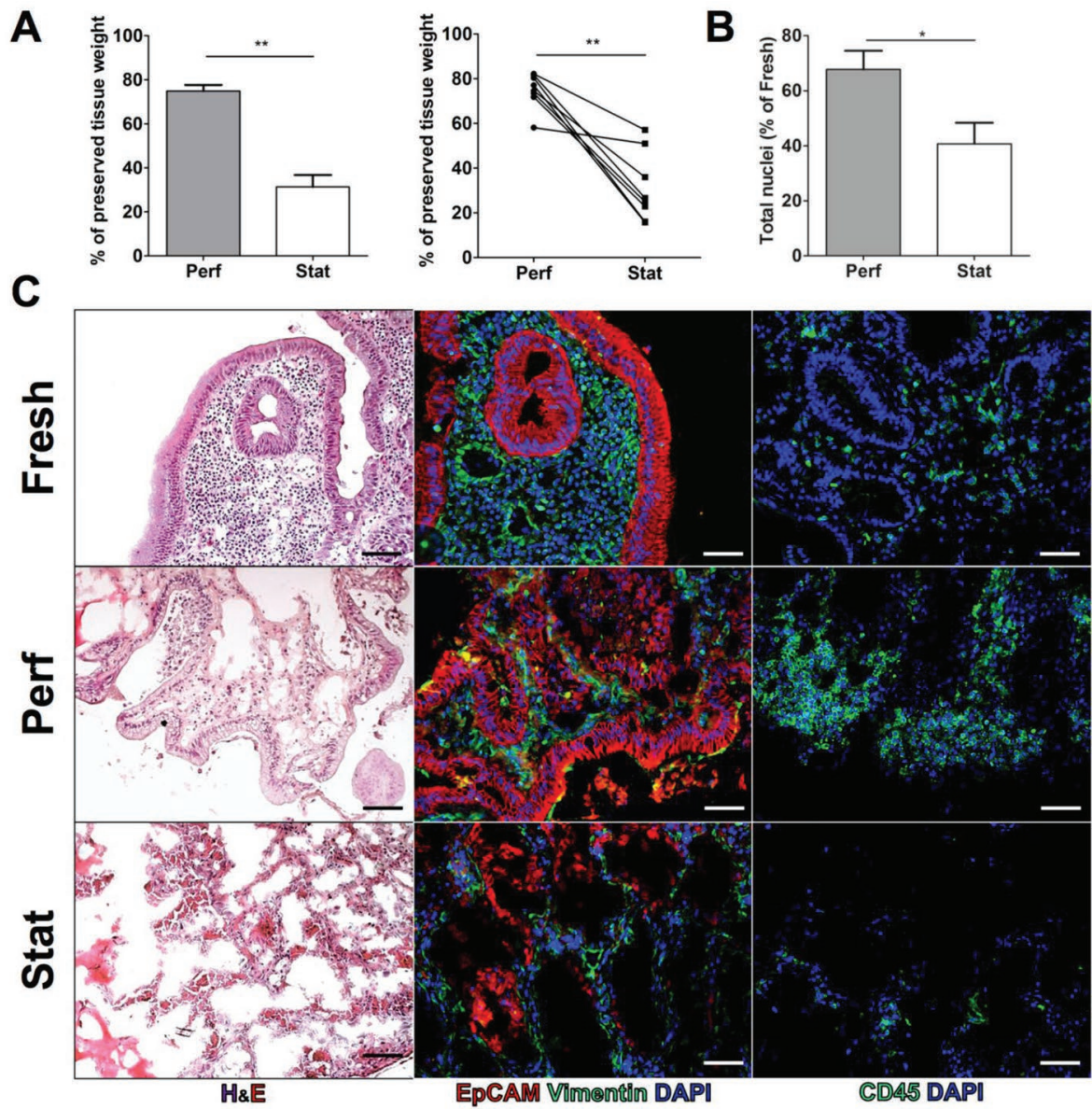

D
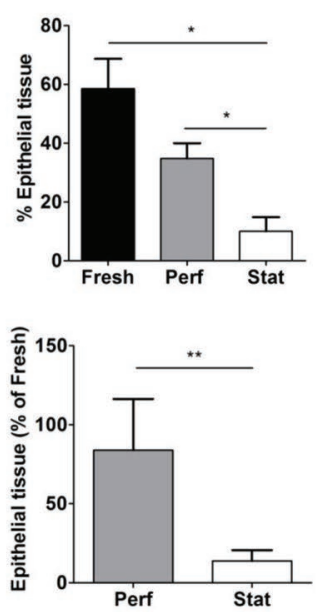

E
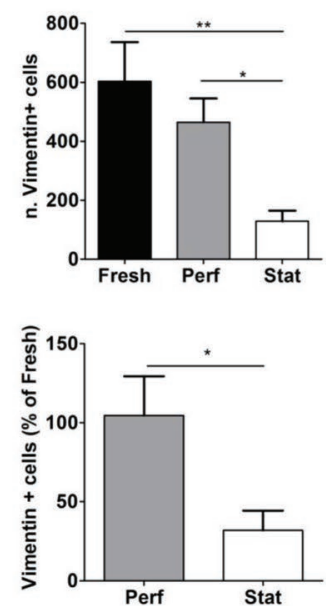

$F$
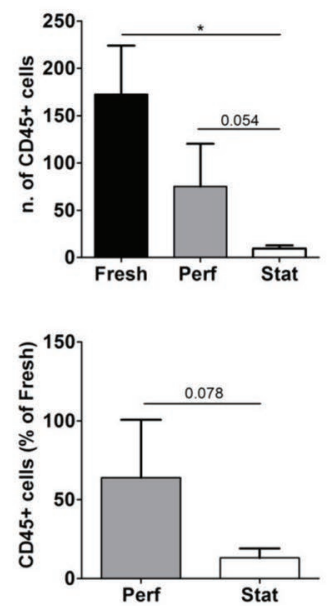

Figure 2. Culture under perfusion preserves the heterogeneity of CRC microenvironment. Tumor fragments derived from CRC specimens were weighted and cultured in perfused bioreactors (Perf) or under static conditions (Stat), as described in Figure 1. After $3 \mathrm{~d}$, fragments were collected, weighted, and embedded in OCT for histomorphological evaluation. A) Weight of cultured tumor chunks is shown as percentage of that recorded prior to culture (fresh). Mean \pm SD ( $n=8$, left panel) and individual values (right panel) are shown. B) Cultured tissues were stained by DAPI and cell nuclei within five ROI per chunk, identified at 40x magnification, were counted using CellProfiler image analysis software. Cumulative data refer to cell nuclei counts in chunks cultured under perfusion or static conditions as related to those of fresh tissues $(n=9)$. Data are expressed as mean \pm SD. C) Fresh tissues or tissues cultured under perfusion or static conditions were subjected to H\&E staining (left panels) or to IF analysis (middle and right panels), following stainings for EPCAM (red), vimentin (green), CD45 (green), and DAPI (blue). Images from a representative CRC sample were obtained at 20X magnification, scale bar $50 \mu \mathrm{m}$. D) Percentages of epithelial tissue scored in fresh or cultured tissues, based on H\&E staining. Cumulative data are expressed as mean \pm SD $(n=7)$. E,F) Numbers of vimentin+ or CD45+ cells counted out of five ROI per chunk are shown $(n=8)$. Statistical significance of differences observed was tested by Wilcoxon signed rank test ( $* p<0.05 ; * * p<0.01)$. 

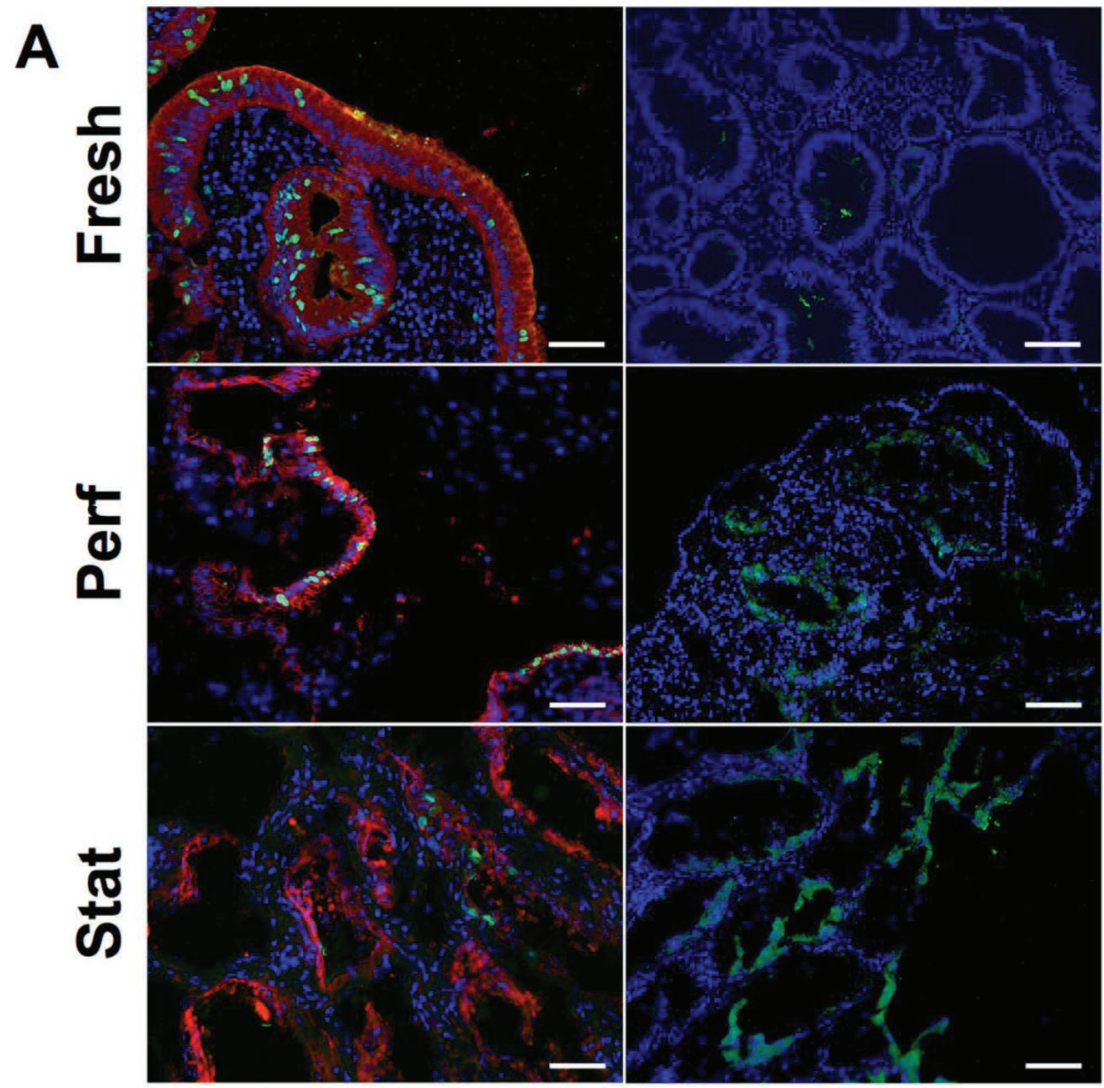

EpCAM KR67 DAPI

GC3 DAPI

B
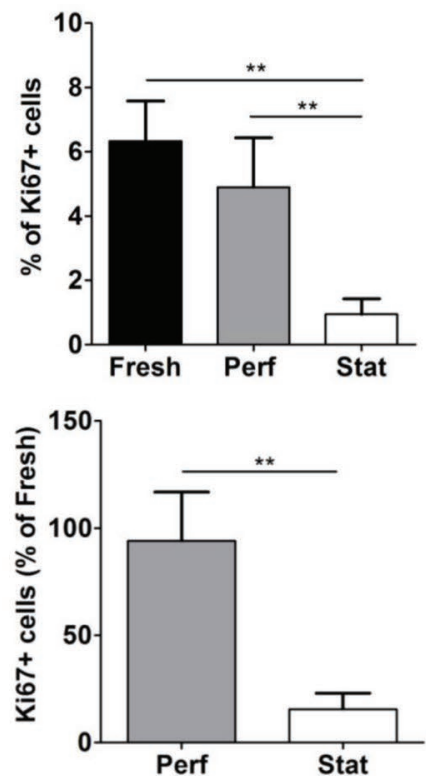

C
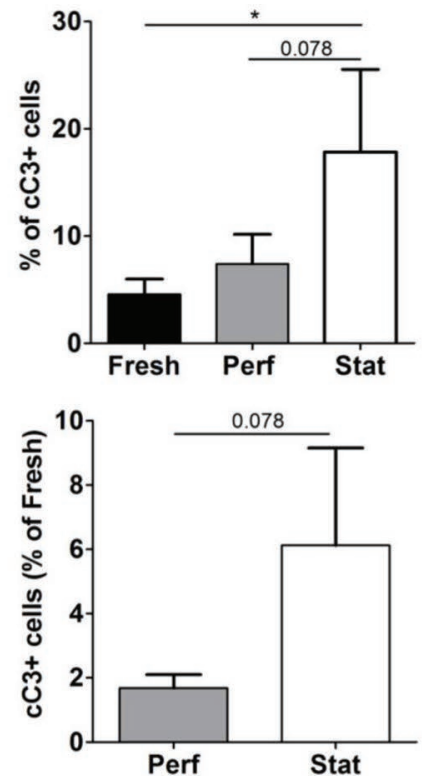

D
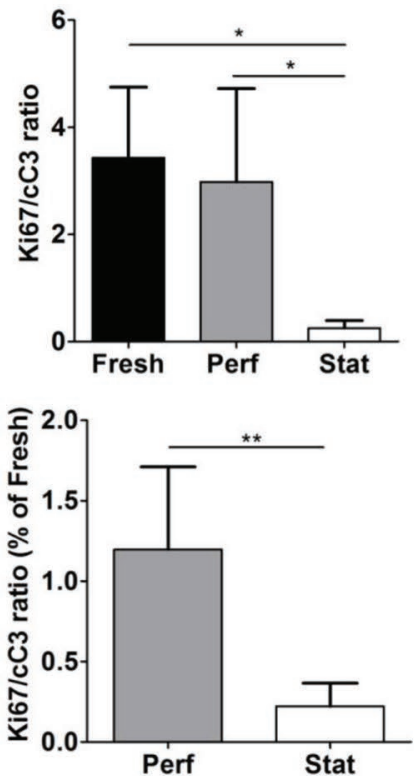

Figure 3. Perfusion allows maintenance of viable and proliferating tumor cells. CRC fragments freshly resected or cultured for $3 \mathrm{~d}$ in perfused bioreactors (Perf) or under static conditions (Stat) were analyzed by immunofluorescence upon staining for Ki67 (green), EpCAM (red), cC3 (green), and DAPI (blue). Five ROI per chunks were identified at 40x magnification, and total cell nuclei were counted using CellProfiler image analysis software. A) Representative images obtained at 20x magnification, scale bar $50 \mu \mathrm{m}$. B-D) Percentages of Ki67+ $(n=9)$ and cC3+ cells $(n=7)$, as related to numbers of total nuclei, and $\mathrm{Ki} 67 / \mathrm{CC} 3$ ratios $(n=7)$ are shown. Cumulative data are expressed as mean \pm SD. Statistical significance of observed differences was tested by Wilcoxon signed rank test ( $* p<0.05 ; * * p<0.01)$. 


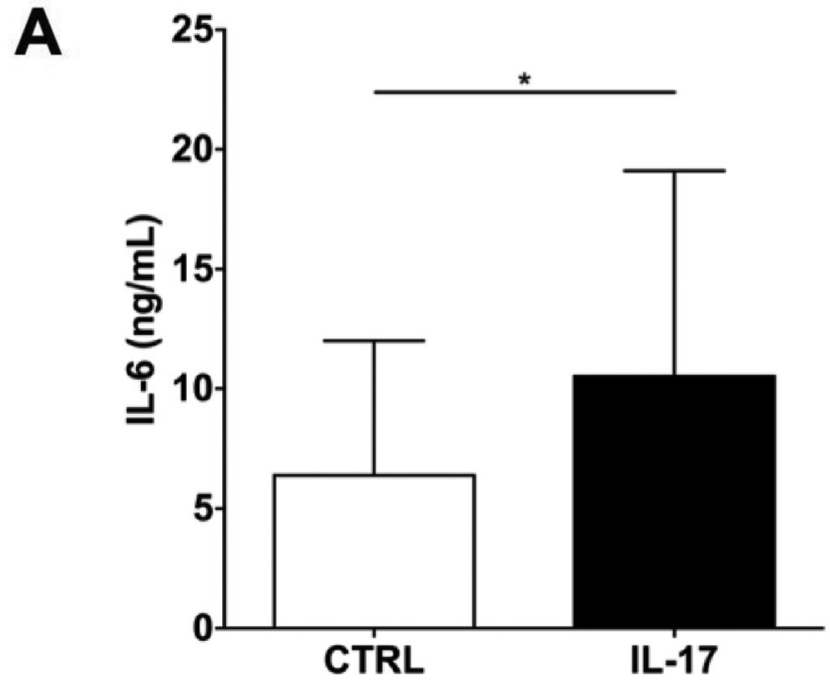

B
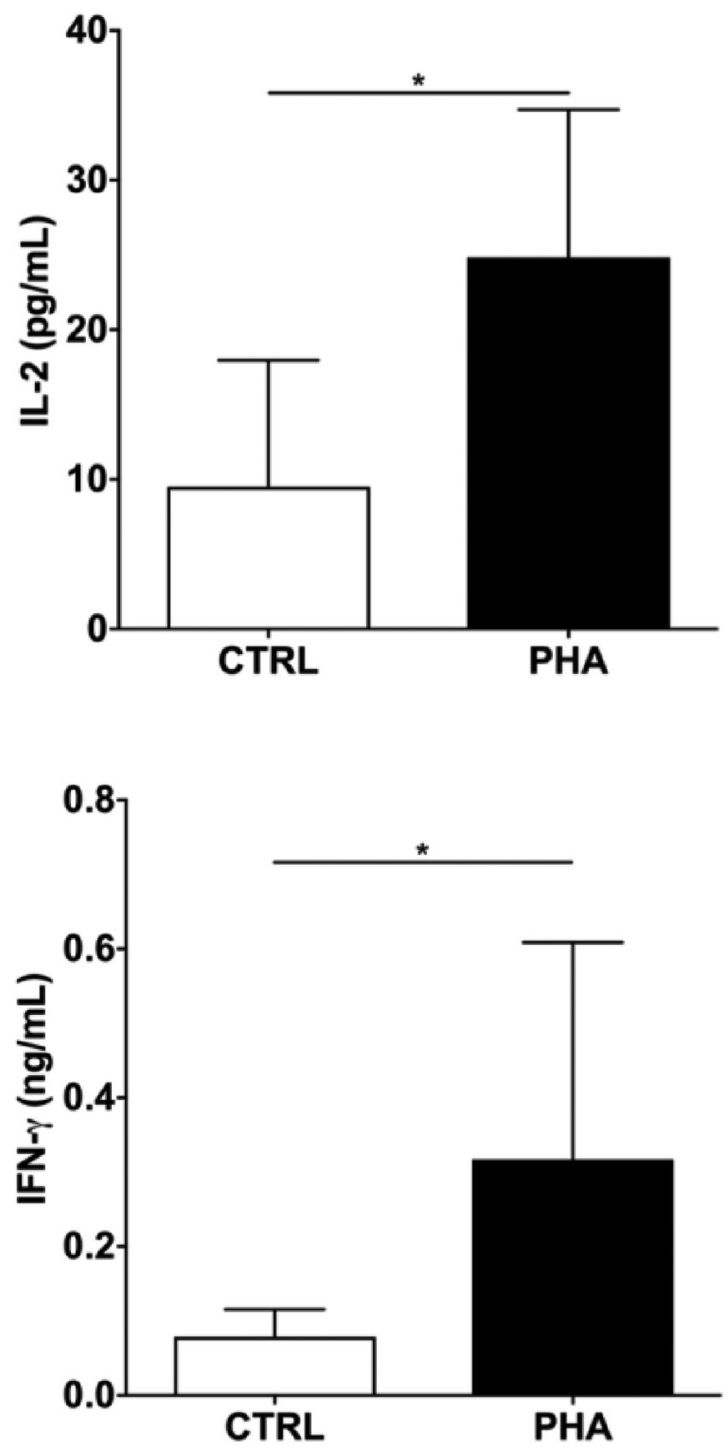

In contrast, in static cultures, percentages of proliferating cells were significantly reduced $(1 \pm 1 \%, p=0.004$ vs both fresh and perfused tissues), whereas those of apoptotic cells were significantly increased $(18 \pm 20 \%, p=0.047$ vs fresh tissues, Figure 3B,C and Figure S5, Supporting Information). Therefore, tissues cultured under static conditions displayed markedly and significantly decreased $\mathrm{Ki67/CC} 3$ ratios (Figure 3D and Figure S5, Supporting Information).

Notably, in all conditions Ki67 positivity was only detected within epithelial tumor cells. Thus, the perfusion allowed maintenance of larger numbers of viable and proliferating tumor cells as compared to static conditions.

Next, we investigated functionality of mesenchymal stromal cells by evaluating their IL-6 release, under steady-state conditions or upon stimulation with IL17, a proinflammatory cytokine promoting IL- 6 secretion by stromal cells. $^{[32]}$

IL-6 release was clearly detectable in media from unstimulated bioreactor cultures. Most importantly, however, its amount was significantly increased upon IL-17 stimulation $(p=0.03$, Figure $4 \mathrm{~A}$ and Figure S6A, Supporting Information), thus indicating that stromal cells maintained in perfused cultures were viable and responsive to microenvironmental stimuli.

We then assessed viability of tumor-infiltrating immune cells cultured under perfusion. In particular, we tested whether infiltrating $\mathrm{T}$ lymphocytes, key players of antitumor immune responses, ${ }^{[11,33]}$ were still responsive to stimulation. Therefore, we measured release of IL- 2 and IFN- $\gamma$ T cell-derived cytokines, upon mitogenic stimulation. As expected, basal levels of $\mathrm{T}$ cell-derived cytokines, in particular of IL-2, largely varied between different samples (Figure S6B, Supporting Information). Importantly, however, they consistently increased upon stimulation (Figure 4B), thus suggesting that immune cells within CRC TME may be successfully activated during culture in bioreactors.

\subsection{Bioreactor-Based Cultures Are Amenable to Test Drug Responsiveness of Primary CRC Tissues}

Finally, we evaluated the possibility to exploit bioreactor-based cultures to test responsiveness to drugs in primary CRC tissues. Fragments from six different samples were cultured under perfusion in the presence or absence of 5-FU, and proliferation and apoptosis rates were evaluated (Figure 5). Following $3 \mathrm{~d}$ of treatment, an overall significant reduction in percentages of epithelial proliferating cells and a significant

Figure 4. Perfused cultures preserve functionality of tumor-associated stromal and immune cells. CRC fragments were cultured within perfused bioreactors for $3 \mathrm{~d}$. A. In the last $24 \mathrm{~h}$ of culture, medium was supplemented with IL-17 (10 $\left.\mathrm{ng} \mathrm{mL}^{-1}\right)$ or left unmodified (CTRL). IL-6 release was assessed by ELISA. Cumulative data are expressed as mean \pm SD $(n=6)$. B) Cultures were performed in the absence (CTRL) or presence of PHA $\left(1 \mu \mathrm{g} \mathrm{mL} \mathrm{m}^{-1}\right)$. Release of IL-2 and IFN- $\gamma$ in culture media was quantified by ELISA. Cumulative data are expressed as mean \pm SD $(n=6)(* p<0.05)$. 


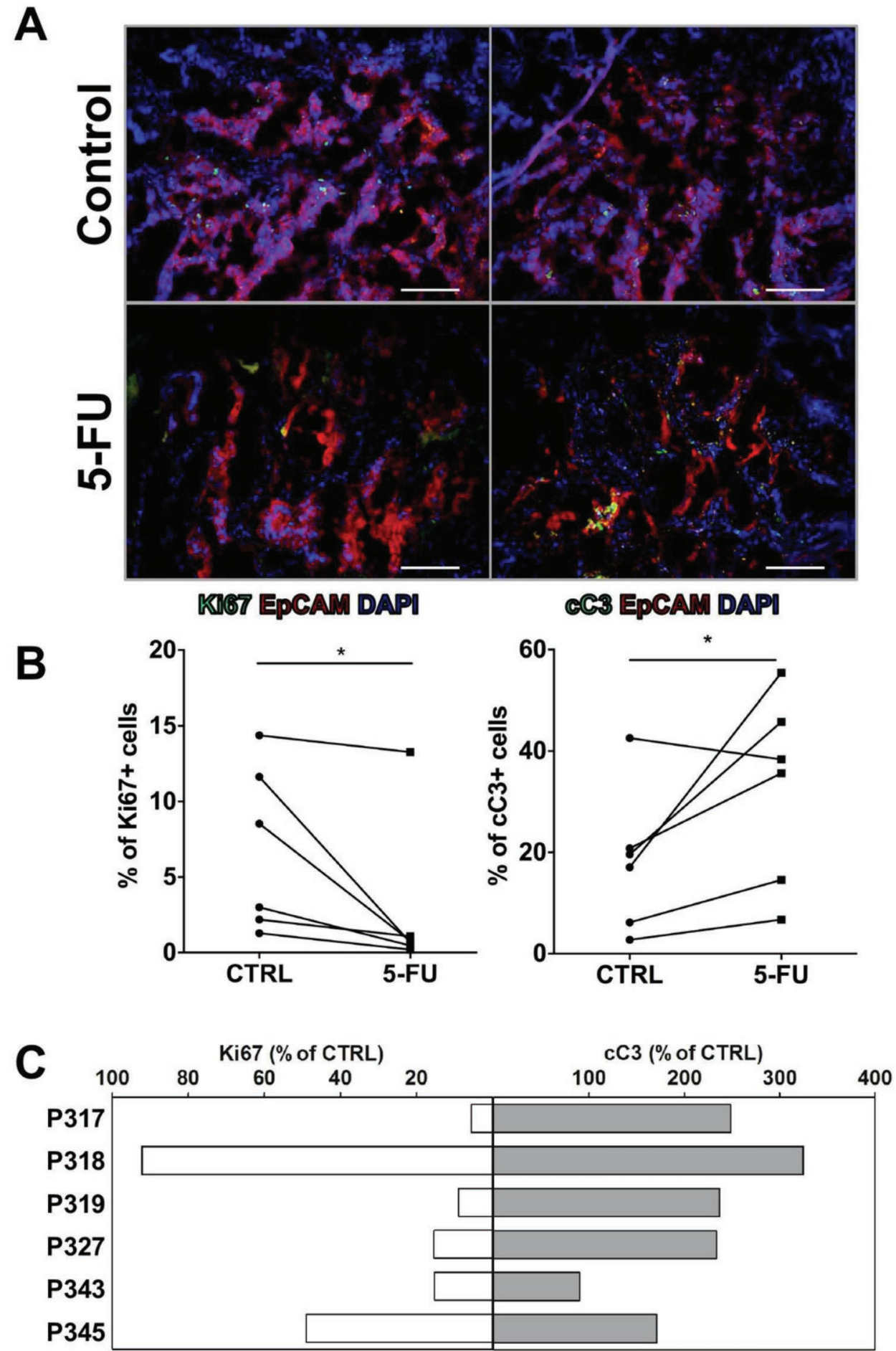

Figure 5. Bioreactor-based cultures are amenable to test drug responsiveness of primary CRC tissues. CRC fragments were cultured within perfused bioreactors for $3 \mathrm{~d}$ in the absence (CTRL) or presence of 5 -FU $\left(10 \mu \mathrm{g} \mathrm{mL} \mathrm{L}^{-1}\right)$. A) Representative IF analysis (magnification $20 \times$, scale bar $\left.50 \mu \mathrm{m}\right)$ upon Ki67 or cC3 (green), EpCAM (red), and DAPI (blue) staining. B) Percentages of Ki67+ (left, $n=6$ ) or cC3+ cells (right, $n=7)$. C) Percentages of Ki67+ and $\mathrm{cC} 3+$ cells in cultures supplemented with 5 -FU relative to untreated tissues in individual samples $(" p<0.05)$.

increase in the fraction of apoptotic cells (Figure 5A,B) could be observed. Notably however, analysis of individual samples revealed more heterogeneous responses. In particular, two out of six samples showed negligible (P318) or partial (P345) inhibition of proliferation, whereas an additional sample (P343) displayed a cytostatic response only, with a dramatic reduction in proliferation but no increase in the percentage of apoptotic cells (Figure 5C). 
Thus, bioreactor-based cultures appear to be suitable to assess patient-specific drug responsiveness in primary CRC within a short time frame.

\section{Discussion}

In the last decade, nontransformed cells of the TME have been recognized to heavily impact on tumor drug responsiveness and clinical outcome. However, in vitro models currently used for drug development do not take into account tumorstromal cell interactions, thus precluding on the one hand to assess the role of nonmalignant cells in tumor drug resistance and on the other to test novel stroma-targeted therapies. This is particularly important for the management of CRC, a leading cause of cancer-related death, for which success rates of conventional chemotherapeutic regimens are limited, whereas alternative therapeutic approaches may be preferable.

In this work, we have shown that culture of freshly resected human CRC specimens in a bioreactor under perfusion results in maintenance of viable whole tumor tissues, including cancer cells together with mesenchymal stromal cells and substantial fractions of immune cells, for a period of time reliably allowing assessment of tumor drug responsiveness.

Tumor cells cultured under perfusion displayed an almost intact structure, as compared to the original tumors, and were viable and proliferating. In addition, stromal cells were maintained in proportions similar to those of original tumors and fully viable, as indicated by responsiveness to microenvironmental stimuli, such as IL-17. Furthermore, immune cells were also partially preserved and were capable of releasing effector cytokines upon activation.

In contrast, in cultures performed under static conditions, fewer viable tumor and stromal cells were preserved, whereas immune cells were completely lost.

Importantly, perfusion-based cultures proved suitable for testing the sensitivity of primary tumor cells to chemotherapies of current use in CRC and revealed heterogeneous responses across individual samples. Thus, our system recapitulates key features of CRC TME and may allow the assessment of tumor response to treatment in a more reliable, patient-specific context.

Established cell lines have long been recognized to substantially differ from primary tumor cells and to fail to mimic the complexity of tumor microenvironment. ${ }^{[34]}$ Therefore, innovative culture systems relying on the use of primary tumor cells have been proposed. In particular, regarding CRC, multicellular tumor spheroids and organoids have been developed. Single CD133+ cells sorted from CRC specimens were shown to be able to expand in vitro in tridimensional spheroid-like structures. ${ }^{[15]}$ However, generation of CRC-derived spheres requires digestion of tumor tissues and presorting of CD133+ cells. Furthermore, sphere formation in CRC only occurs in a limited fraction of samples. ${ }^{[35]}$

Clevers and collaborators have pioneered the establishment of techniques reproducing in vitro "organoids" starting from cell clusters isolated from crypts derived from human normal or neoplastic intestinal epithelium. ${ }^{[17]}$ Organoid formation from primary CRC is highly reproducible and is suitable for high-throughput drug screening. Similarly, CRC tissue-originated spheroids preserving cell-cell contact between primary tumor cells have been shown to expand with high success rates and to be amenable to drug testing. ${ }^{[16,18]}$

However, the formation of both spheroids and organoids relies on the generation of new tumor tissues starting from few putative stem cells. Thus, it requires long culture periods, and the ability of newly generated structures to mirror original tumor tissue features is questionable.

Most importantly, these culture systems, while allowing expansion of epithelial tumor cells, fail to preserve mesenchymal and immune cell TME components, which critically influence tumor growth and responsiveness to treatment in CRC. Thus, they do not allow the assessment of the responsiveness of cancerous cells to anticancer compounds within a context similar to that of in vivo tumors and, in particular, to immunotherapeutic strategies.

Short-term culture (up to $3 \mathrm{~d}$ ) of CRC explants in tumor grade-matched matrix has been proposed as a platform for the prediction of tumor response to chemotherapy. ${ }^{[31]}$ This system certainly represents a major advance in primary tumor culture, inasmuch as it maintains the original tissue architecture and matrix protein components. Indeed, it has been shown to predict response to tumor cell-targeted therapies with high sensitivity and specificity. However, it remains unclear whether this system, where cultures are performed under static conditions, also preserves tumor-associated mesenchymal cells and infiltrating immune cells, thus allowing testing of therapies targeting tumor-associated stroma.

Our work shows that culture of human CRC tissues under perfusion preserves viable tumor cells for a period of time (3 d) comparable to that of the above described study. Most importantly, CRC-associated mesenchymal and immune cells, possibly modulating sensitivity of tumor cells to drugs, are also maintained in a viable and active state. On the other hand, however, the composition of tumor matrix, also possibly influencing CRC survival and drug responsiveness, ${ }^{[36-38]}$ could not be properly assessed in our system. Indeed, although collagen distribution in perfused cultures appeared to be comparable to that of fresh tissues, a proper quantification of matrix components could not be achieved, due to limited tissue availability. We envisage to perform a comparative proteomic analysis between fresh and cultured tissues, either under static or perfused conditions, in the context of followup studies.

The precise contribution of perfusion flow to maintenance of tumor and tumor-associated cells remains to be fully clarified. The continuous direct perfusion allows removal of cell waste products and maintenance of nutrient levels throughout the constructs, which is in turn promoting cellular proliferation and viability. ${ }^{[27,39]}$ Conversely, in static cultures, lack of oxygen and nutrients results in extensive cell necrosis, ultimately leading to proteolytic phenomena. This likely accounts for the dramatic tissue loss observed upon static cultures. Beyond reduction of mass transfer limitations, the flow-associated shear stress has been reported to facilitate the maintenance of stem cell status and prevent spontaneous differentiation. ${ }^{[40]}$ It may thus be speculated that cancer cells with stem cell-like features, including self-renewal ability, are preferentially 
maintained within perfused cultures due to the perfusiondriven mechanical signal, mimicking interstitial fluid flow.

The role of the scaffold composition also deserves to be further addressed in future studies. In this work, we decided to use a collagen-based scaffold, since collagen is a natural polymer and a major component of CRC matrix. ${ }^{[31]}$ In our system, the role of the scaffold was mainly to provide a mechanical support to the tissue chunks, once placed within the perfusion chamber, and, by avoiding flowing of the medium around the chunks, to favor a perfusion as homogenous as possible through the whole chamber. The use of a collagen scaffold did not appear to mediate per se any major effect on tissue maintenance, as in pilot experiments static cultures performed with sandwichscaffolds resulted in a more severe tissue loss than static culture without scaffold (data not shown). However, it would be interesting to test the potential effect of an inert material (e.g., alginate), or of specific ECM components derived from human healthy colonic mucosa or CRC tissues, on survival and proliferation of tumor and stromal cells within cultured tissues.

A proper comparative quantification of the ECM composition in cultured tissues was not possible due to the limited amount of material. A comparative proteomic analysis between fresh and cultured tissues, either under static or perfused conditions, will be attempt.

In conclusion, thanks to its capacity to maintain TME heterogeneity, our system may allow drug testing within a more physiological context. Although not suitable, in its current format, for high-throughput drug testing, our system may be utilized for evaluation of patient-specific sensitivity to available therapeutic options. We envisage validating its ability to predict patientspecific clinical responses in the context of follow-up studies. Furthermore, our culture system may also prove suitable for studying basic aspects of cancer immune-biology or testing therapies whose efficacy is influenced by whole TME (e.g., drug-loaded nanoparticles, ${ }^{[41]}$ targeting mesenchymal or immune cells, including TGF- $\beta$ blockers, ${ }^{[42]}$ bispecific antibodies, ${ }^{[43]}$ or checkpoint inhibitors, ${ }^{[4]}$ currently under clinical investigation for CRC).

\section{Experimental Section}

Tumor Sample Processing: Human primary colorectal cancer specimens were obtained from consenting patients undergoing surgical treatment at the University Hospital Basel, St. Claraspital in Basel, and Ospedale Civico of Lugano, all in Switzerland. Use of human samples was approved by local ethical authorities (Ethikkommission Nordwest und Zentralschweiz, Study Protocol No. 2014-388). Clinico-pathological characteristics of tumor samples used are listed in Table S1 (Supporting Information). Tumor specimens were sampled by experienced gastrointestinal pathologists (L.M.T. and A.Z.) from freshly resected cancer tissues based on macroscopical evaluation, and maintained in Custodiol HTK solution (Dr. Franz Köhler Chemie $\mathrm{GmbH}$ ) at $4{ }^{\circ} \mathrm{C}$ until used. To reduce the risk of contamination due to stool residues, specimens were rinsed in phosphate buffered saline (PBS) before processing, and incubated in a $10 \%$ Octenisept (Schülke \& Mayr AG) solution for $5 \mathrm{~min}$. After additional washings in PBS, tumor specimens were fragmented in $2 \times 2 \times 2 \mathrm{~mm}$ chunks using a scalpel. This size was selected based on preliminary experiments, and showed to allow the perfusion flow through the $8 \mathrm{~mm}$ diameter perfusion chamber, and displayed more limited cutting-related damages. Three randomized chunks from each sample were immediately embedded in optimal cutting temperature compound (OCT, Leica Biosystem) and preserved at $-80^{\circ} \mathrm{C}$, as "Fresh" controls. Remaining fragments were weighted and used for cultures.

Tumor Specimen Culture under Static Conditions: Three randomized CRC fragments were placed into six-well polystyrene culture plates (Sigma) in DMEM/F12 (Gibco) supplemented with $5 \%$ pooled human AB serum (Blood Bank, University Hospital Basel), $2 \times 10^{-3} \mathrm{M}$ Glutamine (GlutaMAX-I, Gibco), $100 \times 10^{-6} \mathrm{M}$ HEPES (Gibco), $1 \mu \mathrm{g} \mathrm{mL}^{-1}$ Kanamycin sulphate (Gibco), $2.5 \mu \mathrm{g} \mathrm{mL} \mathrm{m}^{-1}$ Amphotericin B (Sigma-Aldrich A9528), $20 \mu \mathrm{g} \mathrm{mL} \mathrm{m}^{-1}$ Metronidazol (Braun), $60 \mu \mathrm{g} \mathrm{mL} \mathrm{m}^{-1}$ Cefuroxim (Braun), $10 \mu \mathrm{g} \mathrm{mL}^{-1}$ Ciproxine (Bayer Schering Pharma), $10 \times 10^{-6} \mathrm{M} \mathrm{N}$-acetyl-cysteine (Sigma-Aldrich), $20 \times 10^{-6} \mathrm{M}$ nicotinamide (Sigma-Aldrich), $25 \mathrm{ng} \mathrm{mL}^{-1}$ epidermal growth factor (Stem Cell Technologies), $0.1 \mu \mathrm{g} \mathrm{mL}$ prostaglandin E2 (Tocris Bioscience). $3 \mathrm{~d}$ after, fragments were collected, weighted upon removal of superficial medium by absorbent tissues, and embedded in OCT for subsequent histomorphological evaluation.

Tumor Specimen Culture in Bioreactor under Perfusion: Two scaffold discs $(8 \mathrm{~mm}$ diameter $\times 3 \mathrm{~mm}$ ), made from a porous water-insoluble partial hydrochloric acid salt of purified bovine corium collagen sponge, known as Ultrafoam Collagen Hemostat (Avitene, Bard), were soaked in culture medium for $1 \mathrm{~h}$ at $37^{\circ} \mathrm{C}$.

Three randomized CRC fragments were placed between the discs in a sandwich-like configuration. The sandwich was assembled within a ring-shaped plastic holder closed on top and bottom by two EFTE nylon meshes (Fluorotex Sefar, 09-590/47) (Figure 1). The scaffold assembly was then placed into the perfusion chamber of a previously described perfusion-based bioreactor ${ }^{[30]}$ (currently distributed as U-CUP by Cellec Biotek AG) and perfused with the same culture medium used for static cultures. Perfusion flow rate was set at $0.3 \mathrm{~mL} \mathrm{~min}{ }^{-1}$, corresponding to a superficial velocity of $100 \mu \mathrm{m} \mathrm{s}^{-1},{ }^{[39]}$ as previously used for the generation of normal and tumor tissue-like constructs. ${ }^{[27,30,39,45,46]}$ To assess the functionality of tumor-infiltrating lymphocytes, phytohemagglutinin (PHA, $1 \mu \mathrm{g} \mathrm{mL} \mathrm{m}^{-1}$, Remel Inc.) was added to the culture medium at the beginning of the culture. To evaluate viability and functions of mesenchymal stromal cells, human recombinant IL-17 (10 ng mL ${ }^{-1}, R \& D$ Systems) was added to the culture medium in the last $24 \mathrm{~h}$ of culture. To evaluate responsiveness to drug treatment, 5-FU $\left(10 \mu \mathrm{g} \mathrm{mL} \mathrm{L}^{-1}\right)$ was added to culture medium. In all experiments, a minimum of two bioreactors including a total of six tumor fragments per condition were used.

After $3 \mathrm{~d}$, culture media were collected and assessed for cytokine content by ELISA. Fragments were collected, weighted upon removal of superficial medium by absorbent tissues, and embedded in OCT for histomorphological evaluation.

ELISA: IL-2, IFN- $\gamma$, and IL- 6 release in culture media was assessed by using commercial IL-2 and IFN- $\gamma$ ELISA kits (BD Biosciences), and human IL-6 Instant ELISA (BioLegend), respectively, according to standard protocols.

Histomorphological Assessment and Immunofluorescence (IF): $8 \mu \mathrm{m}$ cryosections were obtained from cryopreserved, OCT embedded, freshly resected tumor chunks, or from fragments maintained in static or perfusion-based cultures. Cryosections were cut from a minimum of two up to ten different levels of the entire tissue block for each specimen, using a $25 \mu \mathrm{m}$ trimming. Slides were fixed with formalin $4 \%$ and stained with hematoxylin and eosin (H\&E) or used for IF analysis.

Upon H\&E staining, abundance of epithelial and stromal components of neoplastic tissues was semiquantitatively assessed by an experienced gastrointestinal pathologist (L.M.T.), based on the evaluation of a minimum of two sections for each specimen and five regions of interest (ROI) per section at 40x magnification using Zeiss Axioskop 2 Plus microscope.

In IF studies, epithelial and stromal cells were identified upon staining with anti-EpCAM mouse mAbs (clone VU1D9, Cell Signaling), followed by Alexa Fluor 546-labeled goat antimouse polyclonal antibodies (A-11030, Invitrogen, 1:100), and anti-vimentin rabbit mAb (clone D21H3, Cell Signaling), followed by Alexa Fluor 488-labeled goat antirabbit polyclonal antibodies (A-11034, Invitrogen). Tumorinfiltrating immune cells were identified by staining with anti-CD45specific Alexa Fluor 488 or Alexa Fluor 647 labeled mouse mAb (clone HI30, BioLegend), anti-CD3 Alexa Fluor 488 (clone UCHT1, BioLegend), 
anti-CD19 Alexa Fluor 594 (clone HIB19, BioLegend), and anti-CD64 Alexa Fluor 647 (clone 10.1, BioLegend). Endothelial cells were identified by staining with anti-CD31-specific DyLight 550 labeled mouse mAb (clone 31.3, Novusbio), and major ECM component collagen type 1 was stained using a rabbit anti-human Collagen 1 (clone EPR7785, abcam). To evaluate proliferating and apoptotic cells, tissue sections were stained with anti-Ki67 488-labeled mAb (clone EPR3610, abcam), or anticleaved Caspase 3 (cC3) rabbit polyclonal antibodies (\#9661, Cell Signaling), followed by Alexa Fluor 488-labeled goat antirabbit polyclonal antibodies (\#A-11034, Invitrogen). Nuclei were counterstained by DAPI (Invitrogen).

Images were taken by using IX83 inverted microscope system (Olympus). Numbers of total nuclei or cells positive for vimentin, cC3, or Ki67 were analyzed using an automated image quantification program (CellProfiler 2.1.1, Broad Institute Imaging Platform). ${ }^{[47]}$ For immune cells and CD31, numbers of total nuclei and positive cells were manually counted. For each specimen, five ROI per section were analyzed.

Statistical Analysis: Statistical significance of observed differences was tested using Wilcoxon matched-pairs signed rank test. Two-sided $p$-values were considered significant at $p$-values $<0.05$ (*p $\leq 0.05 ; * * p \leq 0.01$ ). Statistical analysis was performed using GraphPad Prism version 7.0c (GraphPad Software, La Jolla, CA, www.graphpad.com).

\section{Supporting Information}

Supporting Information is available from the Wiley Online Library or from the author.

\section{Acknowledgements}

C.M., M.G.M., I.M., and G.I. contributed equally to this work. The authors thank Volker Lorber for the initial setup of experiments and Michael Abanto for advice in software-based image analysis. The authors are grateful to Dr. Anna Marsano for critical comments and discussion. All authors have contributed to this work as it follows: C.M., M.G.M., C.H., A.P., G.C.S., I.M., and G.I. conceived and designed the experiments; C.M., M.G.M., V.M., V.G., S.D., S.D.S., R.A.D., R.M., D.O. R.R., M.B., and A.Z. acquired the data; C.M., M.G.M., V.M., V.G., L.M.T., G.C.S., I.M., and G.I. analyzed and interpreted the results; C.M., M.G.M., C.H., V.M., V.G., G.C.S., I.M., and G.I. wrote and critically revised the manuscript; D.O., G.C.S., I.M., and G.I. obtained funding. This work was supported by the Department of Surgery, University Hospital of Basel, and by the Swiss National Science Foundation (Grant Nos. PP00P3-133699 and PP00P3-159262 to G.I. and CRSII5-171037 to I.M.). Patient consent: Obtained. Ethics approval: EKBB/EKNZ (study protocol no. 2014-388). The data generated during and/or analyzed during the current study are available from the corresponding author on reasonable request.

\section{Conflict of Interest}

M.G.M., G.C.S., and I.M. are Cellec Biotek AG shareholders. I.M. is a founder of Cellec Biotek AG. M.G.M., C.H., A.P., and G.I. are inventors of the patent application PCT/EP2015/061614.

\section{Keywords}

3D model, colorectal cancer, human tumor tissue culture, immune cells, perfusion bioreactor, tissue microenvironment

Received: November 5, 2018

Revised: February 3, 2019

Published online: March 7, 2019
[1] J. Ferlay, E. Steliarova-Foucher, J. Lortet-Tieulent, S. Rosso, J. W. Coebergh, H. Comber, D. Forman, F. Bray, Eur. J. Cancer 2013, 49, 1374

[2] D. Cunningham, W. Atkin, H. J. Lenz, H. T. Lynch, B. Minsky, B. Nordlinger, N. Starling, Lancet 2010, 375, 1030.

[3] D. E. Ingber, Differentiation 2002, 70, 547.

[4] J. Schrader, T. T. Gordon-Walker, R. L. Aucott, M. van Deemter, A. Quaas, S. Walsh, D. Benten, S. J. Forbes, R. G. Wells, J. P. Iredale, Hepatology 2011, 53, 1192.

[5] A. L. Correia, M. J. Bissell, Drug Resist. Updates 2012, 15, 39.

[6] A. Calon, E. Lonardo, A. Berenguer-Llergo, E. Espinet, X. Hernando-Momblona, M. Iglesias, M. Sevillano, S. Palomo-Ponce, D. V. Tauriello, D. Byrom, C. Cortina, C. Morral, C. Barcelo, S. Tosi, A. Riera, C. S. Attolini, D. Rossell, E. Sancho, E. Batlle, Nat. Genet. 2015, 47, 320.

[7] C. Isella, A. Terrasi, S. E. Bellomo, C. Petti, G. Galatola, A. Muratore, A. Mellano, R. Senetta, A. Cassenti, C. Sonetto, G. Inghirami, L. Trusolino, Z. Fekete, M. De Ridder, P. Cassoni, G. Storme, A. Bertotti, E. Medico, Nat. Genet. 2015, 47, 312.

[8] A. Calon, E. Espinet, S. Palomo-Ponce, D. V. Tauriello, M. Iglesias, M. V. Cespedes, M. Sevillano, C. Nadal, P. Jung, X. H. Zhang, D. Byrom, A. Riera, D. Rossell, R. Mangues, J. Massague, E. Sancho, E. Batlle, Cancer Cell 2012, 22, 571.

[9] S. Goncalves-Ribeiro, N. G. Diaz-Maroto, M. Berdiel-Acer, A. Soriano, I. Guardiola, M. Martinez-Villacampa, R. Salazar, G. Capella, A. Villanueva, E. Martinez-Balibrea, D. G. Mollevi, Oncotarget 2016, 7, 59766.

[10] W. N. Hait, Nat. Rev. Drug Discovery 2010, 9, 253.

[11] T. N. Schumacher, R. D. Schreiber, Science 2015, 348, 69.

[12] S. Viaud, F. Saccheri, G. Mignot, T. Yamazaki, R. Daillere, D. Hannani, D. P. Enot, C. Pfirschke, C. Engblom, M. J. Pittet, A. Schlitzer, F. Ginhoux, L. Apetoh, E. Chachaty, P. L. Woerther, G. Eberl, M. Berard, C. Ecobichon, D. Clermont, C. Bizet, V. Gaboriau-Routhiau, N. Cerf-Bensussan, P. Opolon, N. Yessaad, E. Vivier, B. Ryffel, C. O. Elson, J. Dore, G. Kroemer, P. Lepage, I. G. Boneca, F. Ghiringhelli, L. Zitvogel, Science 2013, 342, 971.

[13] L. M. Weiner, R. Surana, S. Wang, Nat. Rev. Immunol. 2010, 10, 317.

[14] S. Daster, N. Amatruda, D. Calabrese, R. Ivanek, E. Turrini, R. A. Droeser, P. Zajac, C. Fimognari, G. C. Spagnoli, G. lezzi, V. Mele, M. G. Muraro, Oncotarget 2017, 8, 1725.

[15] L. Ricci-Vitiani, D. G. Lombardi, E. Pilozzi, M. Biffoni, M. Todaro, C. Peschle, R. De Maria, Nature 2007, 445, 111.

[16] J. Kondo, H. Endo, H. Okuyama, O. Ishikawa, H. Iishi, M. Tsujii, M. Ohue, M. Inoue, Proc. Natl. Acad. Sci. USA 2011, 108, 6235.

[17] T. Sato, D. E. Stange, M. Ferrante, R. G. Vries, J. H. Van Es, S. Van den Brink, W. J. Van Houdt, A. Pronk, J. Van Gorp, P. D. Siersema, H. Clevers, Gastroenterology 2011, 141, 1762.

[18] T. Tashiro, H. Okuyama, H. Endo, K. Kawada, Y. Ashida, M. Ohue, Y. Sakai, M. Inoue, PLoS One 2017, 12, e0174151.

[19] C. Hirt, A. Papadimitropoulos, V. Mele, M. G. Muraro, C. Mengus, G. Iezzi, L. Terracciano, I. Martin, G. C. Spagnoli, Adv. Drug Delivery Rev. 2014, 79, 145.

[20] H. Dolznig, C. Rupp, C. Puri, C. Haslinger, N. Schweifer, E. Wieser, D. Kerjaschki, P. Garin-Chesa, Am. J. Pathol. 2011, 179, 487.

[21] S. Nietzer, F. Baur, S. Sieber, J. Hansmann, T. Schwarz, C. Stoffer, H. Hafner, M. Gasser, A. M. Waaga-Gasser, H. Walles, G. Dandekar, Tissue Eng., Part C 2016, 22, 621.

[22] S. Cattin, L. Ramont, C. Ruegg, Front. Bioeng. Biotechnol. 2018, 6, 97.

[23] L. E. Dobrolecki, S. D. Airhart, D. G. Alferez, S. Aparicio, F. Behbod, M. Bentires-Alj, C. Brisken, C. J. Bult, S. Cai, R. B. Clarke, H. Dowst, M. J. Ellis, E. Gonzalez-Suarez, R. D. Iggo, P. Kabos, S. Li, G. J. Lindeman, E. Marangoni, A. McCoy, F. Meric-Bernstam, H. Piwnica-Worms, M. F. Poupon, J. Reis-Filho, C. A. Sartorius, 
V. Scabia, G. Sflomos, Y. Tu, F. Vaillant, J. E. Visvader, A. Welm, M. S. Wicha, M. T. Lewis, Cancer Metastasis Rev. 2016, 35, 547.

[24] S. Julien, A. Merino-Trigo, L. Lacroix, M. Pocard, D. Goere, P. Mariani, S. Landron, L. Bigot, F. Nemati, P. Dartigues, L. B. Weiswald, D. Lantuas, L. Morgand, E. Pham, P. Gonin, V. Dangles-Marie, B. Job, P. Dessen, A. Bruno, A. Pierre, H. De The, H. Soliman, M. Nunes, G. Lardier, L. Calvet, B. Demers, G. Prevost, P. Vrignaud, S. Roman-Roman, O. Duchamp, C. Berthet, Clin. Cancer Res. 2012, 18, 5314.

[25] A. Papadimitropoulos, A. Scherberich, S. Guven, N. Theilgaard, H. J. Crooijmans, F. Santini, K. Scheffler, A. Zallone, I. Martin, Eur. Cells Mater. 2011, 21, 445.

[26] G. Cerino, E. Gaudiello, T. Grussenmeyer, L. Melly, D. Massai, A. Banfi, I. Martin, F. Eckstein, M. Grapow, A. Marsano, Biotechnol. Bioeng. 2016, 113, 226

[27] C. Hirt, A. Papadimitropoulos, M. G. Muraro, V. Mele, E. Panopoulos, E. Cremonesi, R. Ivanek, E. Schultz-Thater, R. A. Droeser, C. Mengus, M. Heberer, D. Oertli, G. lezzi, P. Zajac, S. Eppenberger-Castori, L. Tornillo, L. Terracciano, I. Martin, G. C. Spagnoli, Biomaterials 2015, 62, 138.

[28] M. G. Muraro, S. Muenst, V. Mele, L. Quagliata, G. lezzi, A. Tzankov, W. P. Weber, G. C. Spagnoli, S. D. Soysal, Oncolmmunology 2017, 6, e1331798.

[29] I. Turin, R. Schiavo, M. Maestri, O. Luinetti, B. Bello, M. Paulli, P. Dionigi, M. Roccio, A. Spinillo, F. Ferulli, M. Tanzi, R. Maccario, D. Montagna, P. Pedrazzoli, Med. Sci. 2014, 2, 70.

[30] A. Braccini, D. Wendt, C. Jaquiery, M. Jakob, M. Heberer, L. Kenins, A. Wodnar-Filipowicz, R. Quarto, I. Martin, Stem Cells 2005, 23, 1066.

[31] B. Majumder, U. Baraneedharan, S. Thiyagarajan, P. Radhakrishnan, H. Narasimhan, M. Dhandapani, N. Brijwani, D. D. Pinto, A. Prasath, B. U. Shanthappa, A. Thayakumar, R. Surendran, G. K. Babu, A. M. Shenoy, M. A. Kuriakose, G. Bergthold, P. Horowitz, M. Loda, R. Beroukhim, S. Agarwal, S. Sengupta, M. Sundaram, P. K. Majumder, Nat. Commun. 2015, 6,6169 .

[32] D. Kyurkchiev, I. Bochev, E. Ivanova-Todorova, M. Mourdjeva, T. Oreshkova, K. Belemezova, S. Kyurkchiev, World J. Stem Cells 2014, 6, 552

[33] M. M. Gubin, X. Zhang, H. Schuster, E. Caron, J. P. Ward, T. Noguchi, Y. Ivanova, J. Hundal, C. D. Arthur, W. J. Krebber,
G. E. Mulder, M. Toebes, M. D. Vesely, S. S. Lam, A. J. Korman, J. P. Allison, G. J. Freeman, A. H. Sharpe, E. L. Pearce, T. N. Schumacher, R. Aebersold, H. G. Rammensee, C. J. Melief, E. R. Mardis, W. E. Gillanders, M. N. Artyomov, R. D. Schreiber, Nature 2014, 515, 577

[34] K. M. Yamada, E. Cukierman, Cell 2007, 130, 601.

[35] M. Todaro, M. P. Alea, A. B. Di Stefano, P. Cammareri, L. Vermeulen, F. Iovino, C. Tripodo, A. Russo, G. Gulotta, J. P. Medema, G. Stassi, Cell Stem Cell 2007, 1, 389.

[36] A. Naba, K. R. Clauser, C. A. Whittaker, S. A. Carr, K. K. Tanabe, R. O. Hynes, BMC Cancer 2014, 14, 518.

[37] T. Hoshiba, M. Tanaka, Biochim. Biophys. Acta, Mol. Cell Res. 2016, 1863, 2749.

[38] B. Wei, X. Zhou, C. Liang, X. Zheng, P. Lei, J. Fang, X. Han, L. Wang, C. Qi, H. Wei, Int. J. Biol. Sci. 2017, 13, 1450.

[39] M. Cioffi, J. Kuffer, S. Strobel, G. Dubini, I. Martin, D. Wendt, J. Biomech. 2008, 41, 2918

[40] B. T. Estes, J. M. Gimble, F. Guilak, Curr. Top. Dev. Biol. 2004, 60, 91

[41] A. R. Rama, J. Jimenez-Lopez, L. Cabeza, C. Jimenez-Luna, M. C. Leiva, G. Perazzoli, R. Hernandez, I. Zafra, R. Ortiz, C. Melguizo, J. Prados, Curr. Drug Delivery 2016, 13, 830.

[42] A. de Gramont, S. Faivre, E. Raymond, Oncolmmunology 2017, 6, e1257453.

[43] S. Lehmann, R. Perera, H. P. Grimm, J. Sam, S. Colombetti, T. Fauti, L. Fahrni, T. Schaller, A. Freimoser-Grundschober, J. Zielonka, S. Stoma, M. Rudin, C. Klein, P. Umana, C. Gerdes, M. Bacac, Clin. Cancer Res. 2016, 22, 4417.

[44] L. Nebot-Bral, D. Brandao, L. Verlingue, E. Rouleau, O. Caron, E. Despras, Y. El-Dakdouki, S. Champiat, S. Aoufouchi, A. Leary, A. Marabelle, D. Malka, N. Chaput, P. L. Kannouche, Eur. J. Cancer 2017, 84, 290

[45] M. Santoro, S. E. Lamhamedi-Cherradi, B. A. Menegaz, J. A. Ludwig, A. G. Mikos, Proc. Natl. Acad. Sci. USA 2015, 112, 10304.

[46] M. Radisic, W. Deen, R. Langer, G. Vunjak-Novakovic, Am. J. Physiol.: Heart Circ. Physiol. 2005, 288, H1278.

[47] A. E. Carpenter, T. R. Jones, M. R. Lamprecht, C. Clarke, I. H. Kang, O. Friman, D. A. Guertin, J. H. Chang, R. A. Lindquist, J. Moffat, P. Golland, D. M. Sabatini, Genome Biol. 2006, 7, R100. 


\title{
In Vitro Modeling of Tumor-Immune System Interaction
}

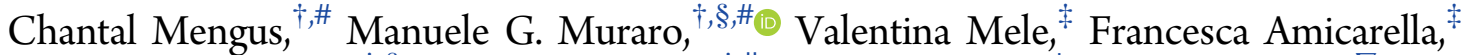 \\ Celeste Manfredonia, ${ }^{\ddagger, \S}$ Federica Foglietta, ${ }^{\dagger, \|}$ Simone Muenst, ${ }^{\perp}$ Savas D. Soysal, ${ }^{\nabla}$ Giandomenica Iezzi, ${ }^{\ddagger}$ \\ and Giulio C. Spagnoli*, ${ }^{\dagger}$ \\ ${ }^{\dagger}$ Oncology Surgery, Department of Biomedicine, ${ }^{\ddagger}$ Cancer Immunotherapy, Department of Biomedicine, and ${ }^{\S}$ Tissue Engineering, \\ Department of Biomedicine, University Hospital Basel, University of Basel, Hebelstrasse 20, 4031, Basel, Switzerland \\ "Department of Drug Science and Technology, University of Torino, Via Pietro Giuria 13, 10125 Torino, Italy \\ ${ }^{\perp}$ Institute of Pathology, University Hospital Basel, University of Basel, Schönbeinstrasse 40, 4056, Basel, Switzerland \\ ${ }^{\nabla}$ Department of Surgery, University Hospital Basel, Spitalstrasse 21, 4031, Basel, Switzerland
}

ABSTRACT: Immunotherapy has emerged during the past two decades as an innovative and successful form of cancer treatment. However, frequently, mechanisms of actions are still unclear, predictive markers are insufficiently characterized, and preclinical assays for innovative treatments are poorly reliable. In this context, the analysis of tumor/immune system interaction plays key roles, but may be unreliably mirrored by in vivo experimental models and standard bidimensional culture systems. Tridimensional cultures of tumor cells have been developed to bridge the gap between in vitro and in vivo systems. Interestingly, defined aspects of the interaction of cells from adaptive and innate immune systems and tumor cells may also be mirrored by 3D cultures. Here we review in vitro models of cancer/immune cell interaction and we propose that updated technologies might help develop innovative treatments, identify biologicals of potential clinical relevance, and select patients eligible for immunotherapy treatments.

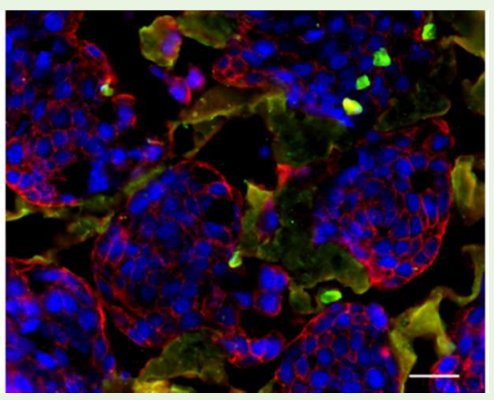

KEYWORDS: tumor infiltrating cells, tumor microenvironment, three-dimensional cultures, tumor engineering, tumor-immune cell interaction

\section{INTRODUCTION}

The interaction between cancer cells and the immune system plays decisive roles in tumor outgrowth and in the control of tumor progression. ${ }^{1}$ Indeed, tumor promoting inflammation ${ }^{2}$ and the ability to escape immune-mediated destruction ${ }^{3}$ do represent bona fide cancer hallmarks. ${ }^{4}$ Studies on clinical specimens have provided a powerful validation of results emerging from experimental models and highly significant prognostic correlations have emerged from the analysis of human tumor infiltration by cells of the innate and adaptive immune system. ${ }^{5}$ Most importantly, immunotherapies now represent routine treatments of patients with cancers of different histological origin. ${ }^{6}$

A variety of monoclonal antibodies (mAbs) have been routinely used for almost two decades in cancer treatment. ${ }^{7}$ In many instances, they were developed to prevent the binding of receptors expressed by tumor cells by growth factors promoting their proliferation. However, mechanisms mediated by immune cells including phagocytosis and antibody-dependent cell cytotoxicity (ADCC) have frequently been shown to underlie their clinical effectiveness. ${ }^{8}$ Indeed, critically depending on their affinity and isotype, ${ }^{9}$ therapeutic mAbs may mediate target cell cytotoxicity elicited by lymphocytes or myeloid cells expressing activating $\mathrm{Fc}$ receptors. A main issue in mAb-mediated immunotherapy, particularly regarding innovative reagents recognizing markers expressed by immune cells, is whether it is desirable to kill target cells or rather to merely inhibit their interaction with specific ligands without killing them. In the latter case, the use of mAbs binding inhibitory Fc receptors would be recommended. Considering current uncertainties concerning the mechanism of action of several therapeutic mAbs, ${ }^{10}$ isotype is emerging as critical for success or failure of reagents recognizing the same target molecule. On the basis of this background, reagents characterized by differential affinity and ability to bind Fc receptors expressed by effector cells are continuously being developed. $^{11,12}$ Moreover, bispecific mAbs specifically targeting defined effector functions to tumor cells are presently in advanced clinical experimentation. ${ }^{13}$

Most importantly, in the past decade, therapeutic mAbs recognizing immunological checkpoints have been successfully tested and utilized in clinical practice. ${ }^{14}$ The rationale underlying their development is that they are supposed to prevent the interaction between activation markers expressed by antigen specific $T$ cells and their ligands expressed by antigen presenting and/or tumor cells, physiologically resulting in the inhibition of adaptive $\mathrm{T}$ cell responses. Releasing the brakes of antitumor responses has proven effective in a variety of cancers. ${ }^{15}$ However,

Special Issue: Tissue Engineering and Biomaterials Approaches to Tumor Modeling

Received: February 3, 2017

Accepted: July 13, 2017

Published: July 13, 2017 


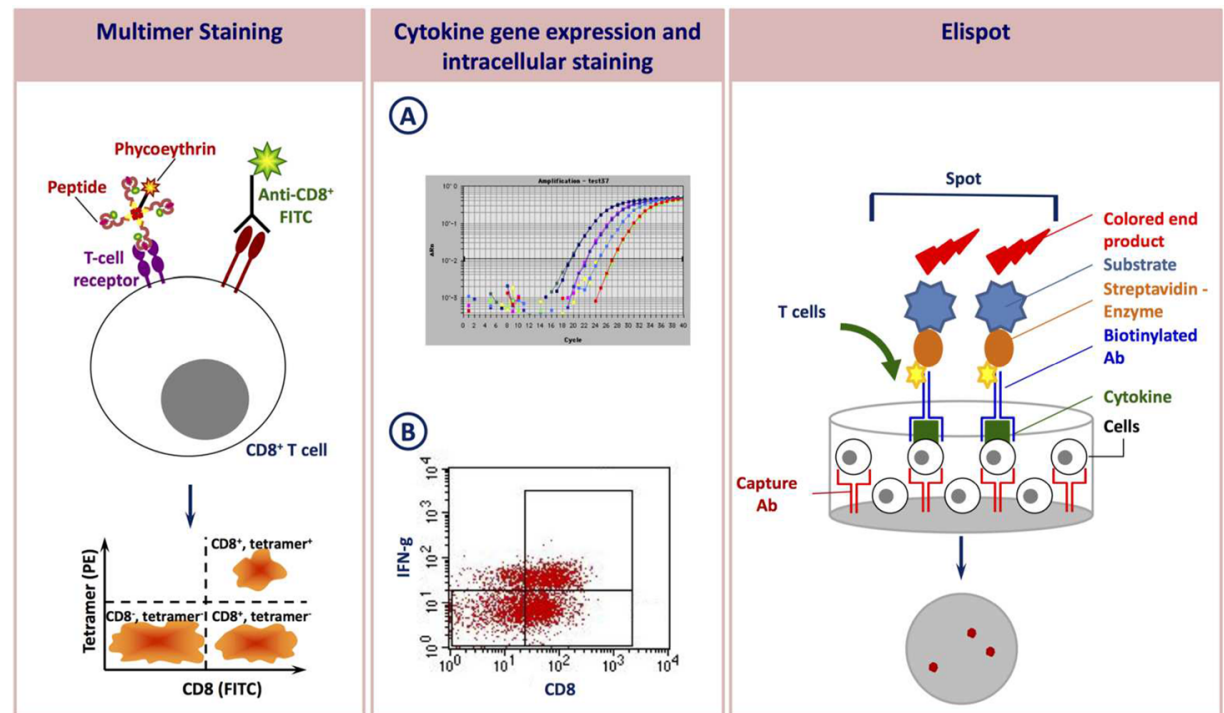

Figure 1. Currently used in vitro assays for the analysis of tumor/T lymphocyte interactions. Antitumor functions of human immune cells are currently assessed in vitro by a variety of established tests. They include the analysis of the expression of T-cell receptors recognizing tumor-specific or tumorassociated antigens (tetramer or multimer staining, left panel). Expression of cytokine genes or production of specific factors upon culture in the presence of tumor cells in standard bidimensional conditions are usually assessed by quantitative PCR (middle panel A) or by flow-cytometry upon intracellular staining (middle panel B). Elispot assays evaluate the numbers of cytokine producing cells, as detectable following culture in the presence of tumor cells or antigen presenting cells pulsed with specific peptides in standard bidimensional conditions (right panel).

mechanisms of action have not been fully clarified and markers predictive of clinical responsiveness still need to be satisfactorily identified. ${ }^{10}$ On a similar line anti-CD $47 \mathrm{mAbs}$ have been used to promote tumor cell phagocytosis by macrophages. ${ }^{16,17}$

Adoptive cancer immunotherapies have also been developed in the past two decades. ${ }^{18}$ They are based on the administration to patients of autologous cells following in vitro culture and expansion. Current adoptive treatments usually capitalize on the use of $\mathrm{T}$ cells from patients transduced with genes encoding conventional or enhanced-avidity HLA-restricted T-cell receptors recognizing tumor-associated antigens, or chimeric HLAunrestricted antigen receptors (CAR) recognizing surface molecules highly expressed by malignant cells. While these technologies are mostly used in the treatment of hematological malignancies ongoing clinical trials also target solid malignancies.

Following these breakthroughs, a large number of innovative biologicals and procedures addressing cancer immunotherapy are being generated and tested in clinical trials and this research field is facing an unprecedented explosion of knowledge and applications, urging the development of adequate assays for preclinical assessments and for the selection of patients potentially benefiting from treatment.

\section{MODELING HUMAN TUMOR-IMMUNE SYSTEM INTERACTIONS: THE PRESENT}

Substantial knowledge underlying the development of therapeutic mAbs and innovative immunotherapy procedures has been gained from in vivo experimental animal models. ${ }^{1,3,19}$ In vitro studies utilizing human cells have proven more problematic, not least due to difficulties inherent in the availability of sufficient numbers of freshly derived tumor or immune cells and of autologous immune/tumor cells systems. Furthermore, the generation of established tumor cell lines from clinical specimens remains a major challenge and the intrinsic heterogeneity of human cancers, in spite of a similar histological origin, must not be underestimated.
Nevertheless, conventional in vitro models have proven of paramount importance in human immunology and, in particular, in tumor immunology. ${ }^{51} \mathrm{Cr}$ release assays ${ }^{20}$ have represented the ultimate tests for the identification of human tumor associated antigens, ${ }^{21,22}$ and standard bidimensional cultures have allowed the expansion of tumor infiltrating lymphocytes, ${ }^{23}$ the generation of tumor specific $\mathrm{T}$ cell clones, ${ }^{22}$ and the monitoring of the effectiveness of therapeutic antitumor vaccinations. ${ }^{24}$ Presently, flow-cytometry techniques based on the detection of cells expressing $\mathrm{T}$-cell receptors recognizing antigenic peptides restricted by defined HLA determinants, for example, multimers, frequently complemented by the analysis of intracellular cytokine expression upon antigenic triggering represent routinely used technologies for the evaluation of adaptive $\mathrm{T}$ cell responses. These techniques are frequently accompanied by so-called Elispot assays identifying individual cells producing specific cytokines upon antigenic stimulation. Combinations of these techniques are currently included in the monitoring of antigen specific $T$ cells responses in patients undergoing immunotherapy treatments (Figure 1). ${ }^{25}$

Cytotoxic activities of NK lymphocytes against malignant cells opsonized by antibody treatments are typically assessed in vitro by using tumor cell line monolayers as targets. Similar assays are also used to analyze the cytotoxic or cytostatic potential of other effector cell types expressing Fc receptors, including macrophages, dendritic cells (DCs), and neutrophils. Tumor cell proliferation or ${ }^{51} \mathrm{Cr}$ release are classically used as read-out. Phagocytosis of tumor cells by macrophages is usually tested by admixing differentially labeled effector and tumor cells in the presence or absence of biologicals of potential therapeutic relevance and using flow-cytometry to identify phagocytosed cells. $^{26}$ 


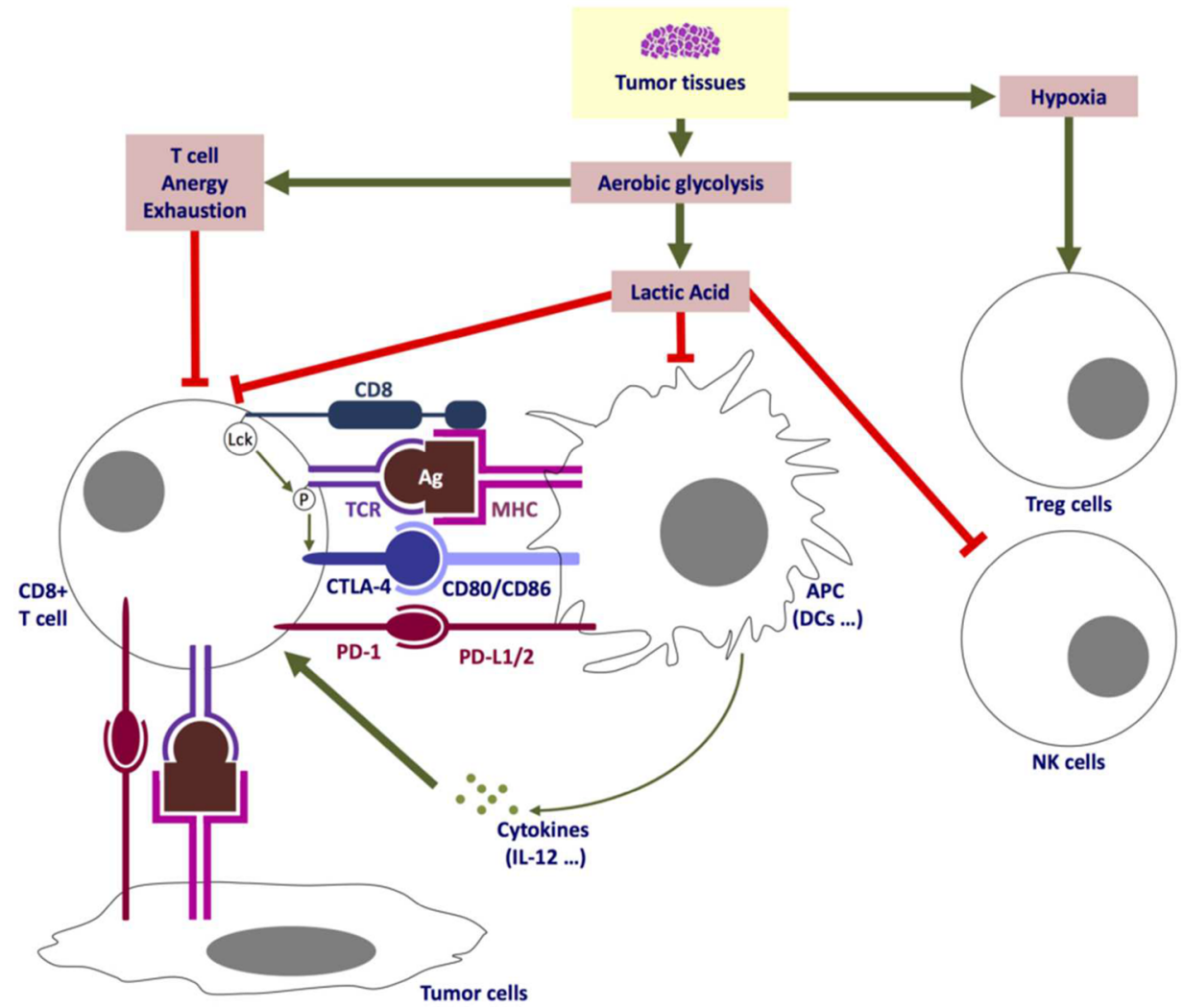

Figure 2. Metabolic alterations of the tumor microenvironment affecting tumor/immune cell interactions. The in vivo tumor microenvironment is characterized by specific metabolic features, including, among others, hypoxia and aerobic glycolysis, resulting in competition for glucose and other nutrients between tumor and immune cells and production of lactic acid. As a result, a variety of effector functions of different immune cell subpopulations are inhibited. Furthermore, functions of antigen presenting cells are also affected. At difference with standard assays, tridimensional culture systems may at least partially mirror these conditions in vitro.

WHY ARE INNOVATIVE MODELS OF TUMOR IMMUNE SYSTEM INTERACTION IMPORTANT?

In vitro data consistently indicate that, in defined assay conditions, at least $\mathrm{T}$ and $\mathrm{NK}$ lymphocytes and macrophages are able to efficiently elicit antitumor functions. Notably, however, cytotoxic tumor infiltrating $\mathrm{T}$ lymphocytes are frequently disfunctional in vivo, ${ }^{27}$ as also indirectly suggested by the clinical effectiveness of immunological checkpoints targeted treatment. ${ }^{28}$ Furthermore, immune-histochemical studies suggest that solid tumors most frequently lack detectable NK cell infiltration. ${ }^{29,30}$ More importantly, with a few exceptions, including colorectal cancer (CRC), macrophage infiltration of solid tumors is usually associated with poor prognosis. ${ }^{31}$

Discrepancies between in vivo and in vitro functional profiles of immune cells have stimulated research aimed at unraveling mechanisms and conditions favoring $\mathrm{T}$ cell anergy and exhaustion, pro-tumor macrophage polarization, defective NK cell recruitment and, ultimately, tumor escape from immune surveillance. A variety of different cell types including alternatively activated macrophages, ${ }^{32}$ regulatory $\mathrm{T}$ cells (Treg), ${ }^{33}$ and myeloid derived suppressor cells ${ }^{34}$ have been considered. Furthermore, immunosuppressive mechanisms at work in the tumor microenvironment have been shown include hypoxia and adenosine receptor triggering, ${ }^{35,36}$ and expression of ligands for immunological checkpoints (see above).

Earlier reports in the past had suggested that oxygen levels may dramatically affect lymphocyte responsiveness. ${ }^{37}$ More recently, a large number of important studies appear to indicate that hypoxia and specific metabolic conditions occurring with tumor tissues might provide a unifying background for a variety of previously observed immunosuppressive mechanisms and decisively hamper the potential effectiveness of anticancer immune responses. Indeed, hypoxia has been shown to promote immune tolerance by Treg recruitment. ${ }^{38}$ Intriguingly, expression of PD-1 immunological checkpoint has been related to metabolic alterations occurring within tumor tissues. ${ }^{39,40} \mathrm{~A}$ key point appears to be represented by the competition for glucose between tumor cells and T-cell receptor triggered, antigen specific $\mathrm{T}$ cells, both characterized by aerobic glycolysis. ${ }^{41-44}$ Moreover pro-tumor M2 macrophage activation has also been associated with increased glycolysis, ${ }^{45,46}$ and the development of myeloid derived suppressor cells within the tumor microenvironment has been related to hypoxia (Figure 2). ${ }^{47}$

While these phenomena have been extensively characterized in vivo and ex vivo, although mostly in experimental models, they also suggest the fascinating possibility of generating innovative in vitro models adding new dimensions to the analysis of the tumor microenvironment in highly controlled conditions and allowing the preclinical screening of biologicals and small molecules in conditions closer to in vivo features of the human tumor microenvironment. 

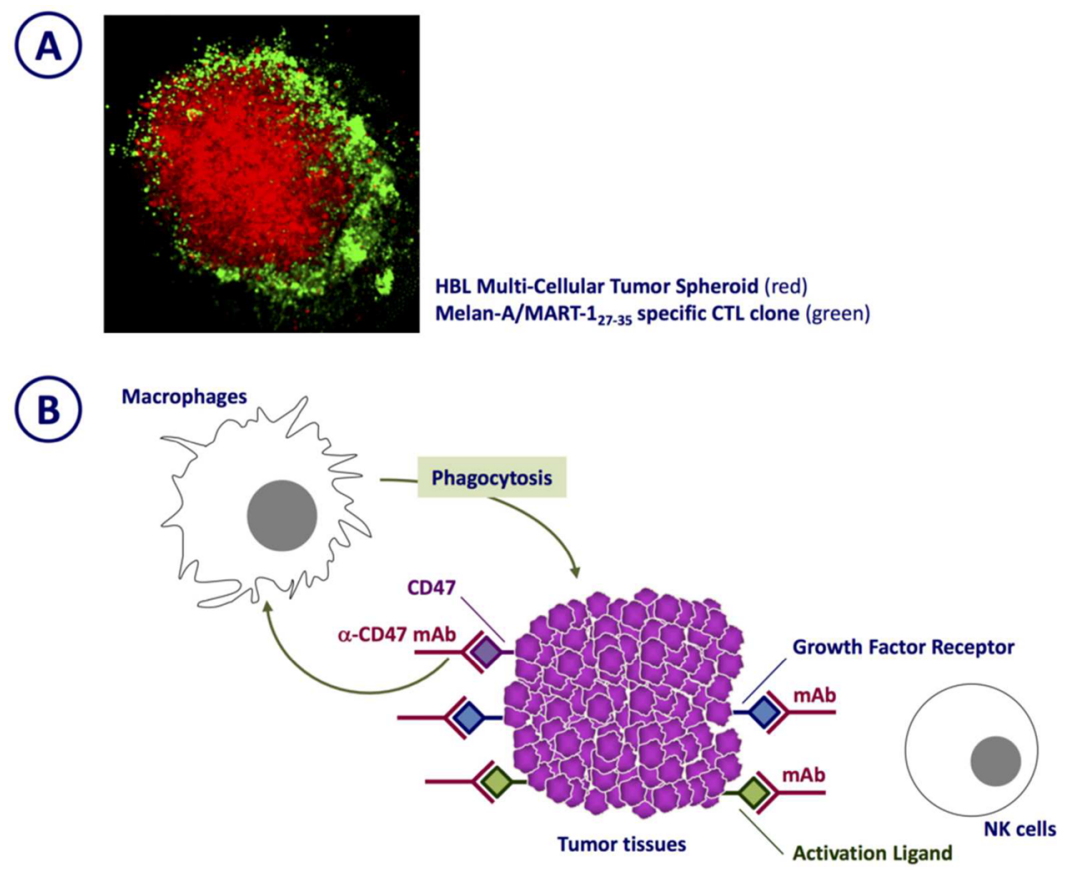

Figure 3. Tumor cell spheroids as targets of immune cell effector functions. Tumor cell spheroids generated by different procedures have been used to verify the effects of culture in tridimensional conditions on a variety of immune cell functions. T-cell clones recognizing melanoma-associated antigens have been cocultured with melanoma cells (panel A). CAR-transduced cells for adoptive treatments have similarly been tested. Functions of monocyte/ macrophage lineage cells, including phagocytosis and antigen presentation have also been assessed. Moreover, antibody-dependent cell cytotoxicity mediated by NK cells has been explored using target cells cultured as spheroids (panel B).

\section{MODELING HUMAN TUMOR-IMMUNE SYSTEM INTERACTIONS: THE THREE-DIMENSIONAL APPROACH}

To address the high attrition rate in the development of innovative anticancer compounds a variety of tridimensional culture models have been developed in the past. ${ }^{48}$ They have revealed the major role played by the architecture of cell growth in the definition of the gene expression profiles of tumor cells, their metabolic activities, and their sensitivity or resistance to drug treatment. ${ }^{49-51}$ On the basis of these findings, innovative high throughput drug screening platforms have been generated and are currently utilized in pharmacological research. In initial studies, multicellular spheroids were obtained by preventing the adhesion of tumor cells on plastic cell culture surfaces. ${ }^{52}$ Later, scaffolds, hanging drops, and microfluidics-based technologies were successfully developed. ${ }^{53}$

Control of spheroid size has allowed the generation of structures characterized by controlled levels of hypoxia and perfused bioreactors have proven to be useful to generate tissuelike structures from established human tumor cell lines. ${ }^{54,55}$ In this context, it is also remarkable that human cancer cells endowed with tumor initiating capacity, so-called tumor initiating cells (TIC) or cancer stem cells (CSC), from tumors of different histological origin, including colon, breast, and CNS, are typically characterized by the ability of generating spheres that are able to slowly replicate with asymmetric divisions. ${ }^{56,57}$

Models of higher complexity are continuously being developed $^{58,59}$ aiming at including additional components of the tumor microenvironment of proven relevance in clinical course and in the development of resistance to treatment. Furthermore, physical conditions within tumor tissues and the possibility of reliably reproducing them in vitro are increasingly attracting the attention of the scientific community. In particular, microfluidics models have been generated ${ }^{60}$ to address sensitivity to drugs and dissemination of cancer cells, ${ }^{61}$ tumor lymphatic vessel interaction, ${ }^{62}$ and homing of tumor cells to defined metastatic niches. ${ }^{63}$ Intriguingly, however, the first 3D culture models had initially been developed to address immune responsiveness to solid tumor allografts. ${ }^{52}$

In view of this background it is surprising that only relatively few studies have addressed the effects of 3D culture of tumor cells and on their sensitivity to lymphocyte effector activities. Pioneering works suggested that tumor cells cultured in 3D were poorly targeted by cytokine activated lymphocytes ${ }^{64}$ and that the disruption of these architecture represented an important prerequisite for a full elicitation of antitumor cytotoxicity. ${ }^{65}$ More recently, we and others observed that $\mathrm{T}$ cell effector functions are severely impaired when target cells are structured in 3D architectures. ${ }^{66,67}$

Different mechanisms have been proposed. Dangles-Marie et al. suggested that decreased expression of heat shock protein-70 by tumor target cells might result in inefficient antigen presentation. ${ }^{68}$ We observed that cells from established melanoma cell lines may down-regulate expression of HLA and melanoma differentiation antigens following culture in spheroids. ${ }^{69}$ Interestingly, decreased expression of Melan-A/MART1 differentiation antigen has also been observed in hypoxic areas of clinical melanoma specimens. ${ }^{70}$

On the other hand, lactic acid is produced to increasing extents in cells cultured in 3D, as compared to their $2 \mathrm{D}$ counterparts. ${ }^{69,71}$ Notably, concentrations of lactic acid produced in these conditions are sufficient to significantly inhibit the elicitation of effector functions of antigen specific cytotoxic $\mathrm{T}$ lymphocyte (CTL) clones, thus providing an important link between typical metabolic features of tumor cells and $\mathrm{T}$ cell functional impairment. 
NK lymphocyte infiltration has also been studied in scaffoldfree and 3D Matrigel-based models ${ }^{72,73}$ and the impaired cytotoxic ability of natural killer (NK) cells against targets cultured in tridimensional architectures has also been reported. ${ }^{74}$ In particular, the resistance of tumor cells to NK lymphocytemediated cytotoxicity in 3D glioma models has been attributed to increased HLA-E expression by tumor cells. ${ }^{75}$ NK and Treg interaction with breast cancer cells in $3 \mathrm{D}$ has been shown to result in increased production of CCL4-attracting inflammatory cells of pro-tumor significance. ${ }^{73}$ Instead, despite their potential relevance in the cancer microenvironment, there is a lack of studies investigating B-cell tumor cell interaction in 3D architectures. Most recently, models based on microfluidic technology have also been proposed to analyze tumor/ lymphocyte interaction. ${ }^{76}$

Interestingly, recently, an advanced model based on hanging drop technology and including fibroblasts, additional key components of the tumor microenvironment has been successfully used to explore the ability of different types of immune cells to display their effector, antitumor potential, ${ }^{77}$ as mediated by therapeutic mAbs.

A number of studies on tridimensional modeling have focused on lymphocytes. However, macrophages and other myeloid cells are also frequently infiltrating human cancers. ${ }^{78}$ Murine and human cells of the monocyte/macrophage lineage may be polarized by cytokine treatment into M1 macrophages endowed with antitumor potential or M2 macrophages which have been shown to be rather tumor-supportive and characterized by a proangiogenic functional profile. ${ }^{32}$ It is worth noting that the M1/ M2 polarization notion represents a useful oversimplification of a process more realistically described as a continuum. ${ }^{79}$ Nevertheless, the culture of monocytes and macrophages within tridimensional tumor spheroids has been shown to profoundly affect their differentiation and functional profiles. ${ }^{80-82} \mathrm{~A}$ coculture of human and murine macrophages together with squamous cell carcinoma cells in $3 \mathrm{D}$ architectures, in the presence or absence of fibroblasts, has been shown to promote their polarization toward an M2 functional profile and induce metalloproteases (MMP) production, thereby favoring tumor invasiveness, as related to increased extracellular matrix degradation. ${ }^{83}$ Similar observations were also made in experiments performed by using breast, ${ }^{84,85}$ thyroid, ${ }^{86}$ hepatocellular, ${ }^{87}$ and bladder ${ }^{88}$ cancer cell lines. In all these cases alterations of the chemokine secretome in $3 \mathrm{D}$ cultures including tumor cells and macrophages in the presence or absence of fibroblasts were consistently observed. NSCLC cells cultured in aggregates have been shown to preferentially attract M2 macrophages, which, in turn promote their epithelial-mesenchymal transition (EMT) and migration, as observed by using microfluidic devices. ${ }^{61}$ In this study macrophages cultured in different conditions, potentially related to intermediate polarization stages were comparatively analyzed. Most recently, tumor cell migration in a 3D extracellular matrix was also reported to be enhanced by macrophage-secreted TNF $\alpha$ and TGF $\beta 1{ }^{89}$

On the other hand, importantly, antigen presentation and differentiation capacity of DCs have been shown to be inhibited by lactic acid produced by tumor cells in $3 \mathrm{D}$ cultures including microfluidic models (Figure 3). ${ }^{71,90}$ These data indicate that tridimensional models could also be advantageously used to analyze, in controlled conditions, the interactions occurring in vivo between tumor cells and cells of the monocyte/macrophage/dendritic cell lineages (Table 1).
Table 1

\begin{tabular}{|c|c|c|}
\hline & 3D culture system & ref \\
\hline \multirow{2}{*}{$\begin{array}{l}\text { cytotoxic T } \\
\text { lymphocyte } \\
\text { activity assays }\end{array}$} & spheroids & $52,64,66,67$ \\
\hline & engineered tumor models & 76,110 \\
\hline $\begin{array}{l}\text { NK cytotoxicity } \\
\text { assays }\end{array}$ & spheroids & 74 \\
\hline $\begin{array}{l}\text { monocytes/ } \\
\text { macrophage/DC }\end{array}$ & spheroids & $71,80,81,116$ \\
\hline $\begin{array}{l}\text {-tumor cell } \\
\text { interaction }\end{array}$ & microfluidic devices & 60,90 \\
\hline $\begin{array}{l}\text { therapeutic mAbs } \\
\text { (ADCC, Bispecific } \\
\text { Abs) }\end{array}$ & spheroids & $\begin{array}{l}12-14,65 \text {, and } \\
77\end{array}$ \\
\hline $\begin{array}{l}\text { drug tests in } \\
\text { engineered } \mathrm{TME}^{a}\end{array}$ & $\begin{array}{l}\text { in vitro engineered tissue models } \\
\text { (spheroids, microfluidics devices } \\
\text { bioreactors) }\end{array}$ & $\begin{array}{l}51,54,55,58, \\
59,117 \text {, and } \\
118\end{array}$ \\
\hline
\end{tabular}

Interestingly, neutrophil polarization similar to functional features similar to those detected in macrophages, has also been recently reported. ${ }^{82,91}$ However, possibly due to difficulties inherent in a granulocyte culture, the effects of incubation with tumor cells cultured in 3D on their polarization have not been addressed so far, and further research in this area is warranted.

\section{MODELING HUMAN TUMOR-IMMUNE SYSTEM INTERACTIONS: THE BIOMATERIALS}

In addition to cell composition and structural architecture, the extracellular matrix (ECM) also plays key roles in the tumor microenvironment, critically affecting cancer cell dynamics and response to treatment in vivo and in vitro. ${ }^{92,93}$ To address these issues, a variety of biomaterials are currently being evaluated to help mimic tumor microenvironment features. While a thorough analysis of biomaterials used in 3D cultures of tumor cells ${ }^{94}$ clearly exceeds the purposes of this review, it might be of interest to recapitulate recent advances in this area, as related to the modeling of tumor-immune system interaction.

The use of a decellularized matrix ${ }^{95}$ from cancer specimens has been proposed. ${ }^{96}$ However, harsh decellularization treatments might result in loss of ECM components and alterations of its ultrastructure. ${ }^{95}$ Furthermore, ECM from human tissues are not commercially available. Notably, ECM composition may be remarkably different in cancers of similar histological origin, thus complicating standardization. For instance, in CRC, while collagen type 1 is the single most represented ECM component, laminin and fibronectin may also be present to highly different extents in different samples. ${ }^{97}$ Useful simplifications of these complex issues might reside in the use of single most represented components $^{98}$ or commercially available ECM mixtures from experimental animals, such as Matrigel or Cultrex. ${ }^{86,99}$ Even in these cases, however, differences from batch to batch of commercial products should not be underestimated. Agar, agarose, and hyaluronic acid have also been used for spheroid formation. ${ }^{100}$

In a number of reports the tumor-immune system interaction in $3 \mathrm{D}$ structures has been investigated in the absence of scaffolds. ${ }^{66-69,72,88}$ In these studies spheroids might be righteously considered as building blocks of in vitro developed tumor tissues, also considering the ability of cancer cells to produce ECM components. Alternatively, collagen has been used as scaffold or to coat microfluidics devices. ${ }^{61,83,89,101}$ Matrigel and Cultrex have been widely utilized ${ }^{85,86,102}$ and the use of alginate ${ }^{84}$ and synthetic materials has also been investigated. ${ }^{103,104}$ 


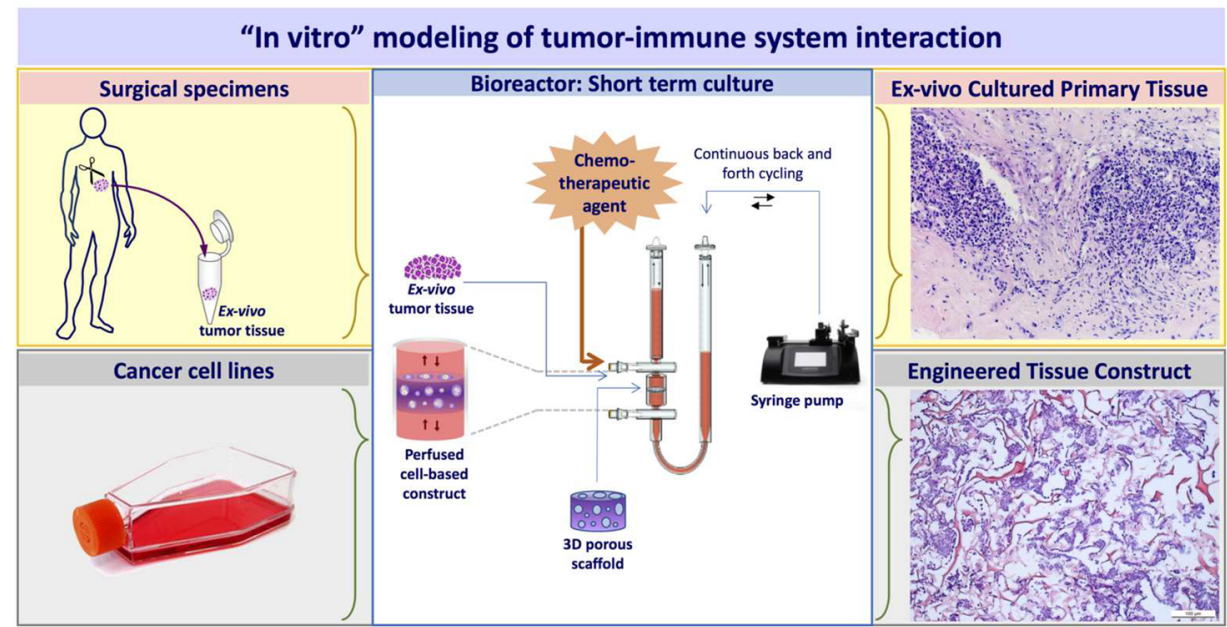

Figure 4. Innovative tridimensional models of tumor/immune cell interaction. Innovative models of tumor immune system interaction may take advantage of the use of established cell lines producing tissue-like structures upon culture in perfused bioreactors. Furthermore, the use of ex vivo cultured fragments from surgically excised cancers could also be envisaged. In either case, combinations of immune cells, biologicals and/or small molecules could be tested for their effects on malignant cells.

On the other hand, progress in the characterization of natural biomaterials and in the engineering of synthetic ones, combined with advances in the understanding of biological processes, have widely extended the range of compounds under investigation. ${ }^{94}$ Multifunctional biomaterials targeting defined cell populations and favoring cell-to-cell interactions and crosstalk have been designed. Some of them are able to promote durable immune responses by protecting agents from degradation and providing sustained signals to host immune cells. ${ }^{105-108}$ Therefore, biomaterials are evolving from mere structural supports into tools interacting with cells and tissues to induce and modulate biological responses.

It is tempting to speculate that $3 \mathrm{D}$ models of cancer-immune cell interaction will prove extremely useful for the preclinical testing of innovative biomaterials.

\section{MODELING HUMAN TUMOR-IMMUNE SYSTEM INTERACTIONS: AN OUTLOOK}

Tumor tissues include a large variety of nonmalignant cells. Their numbers may vary widely depending on the histological origin of the cancer. For instance, in melanoma, cancer cells usually account for $>90 \%$ of the cells detectable within clinical specimens. In contrast, malignant cells represent a mere $10 \%$ of cells from cancer tissues in Hodgkin lymphoma. The mutual interaction between malignant and nontransformed cells is highly dynamic and critically affects both components of the tumor microenvironment. ${ }^{109}$ In the recent past, engineered tumor tissue constructs have successfully been used to investigate the chemo-attractive potential of tumor and tumor infiltrating cells. $^{110}$

Most importantly, the composition of the tumor microenvironment is of decisive relevance to predict the clinical course of the disease $\mathrm{e}^{111,112}$ and the response to treatment. ${ }^{113}$ This background urges the development of techniques allowing the investigation of functional features of the human tumor microenvironment in controlled conditions. However, a number of hurdles need to be preliminarily addressed. For many human cancers, no reliable experimental model is available. Moreover, the characteristics of the immune systems of a variety of inbred murine strains poorly mirror those detectable in patients' populations. ${ }^{114}$ On the other hand, generation of established cell lines from clinical specimens is only feasible in a limited number of human cancer types.

To obviate these difficulties the generation of patient-derived xenografts (PDTX) in immune-deficient mice has been proposed for personalized assessment of the sensitivity of tumor cells to defined chemotherapy regimens. ${ }^{115}$ These assays are widely used in basic and translational research. However, they are characterized by a number of limitations. In vivo growth of xenografts might be difficult or require relatively long time spans, particularly for tumors of specific histological origin, such as prostate cancers. In addition, human tumor cell growth might be limited by the lack of cross-species activity of a variety of factors produced in the xenograft microenvironment. Most importantly, PDTX technologies are poorly suitable for the evaluation of biologicals and small molecules targeting tumor-immune system interaction, since human interstitial cells are rapidly replaced by murine cells in successfully growing xenografts, and human infiltrating immune cells are lost.

Ideally, innovative assays should include as many cellular components of the microenvironment of a specific cancer as possible. This represents a major challenge since primary and metastatic tumor niches may be substantially different. Furthermore, even in cancers of similar histological origin, the tumor microenvironment is highly variable and its composition might also be related to factors, for example, commensal flora in colorectal cancers poorly amenable to in vitro modeling.

To attempt to address these issues, at least in part, Majumder et al. used entire fragments of clinical specimens to predict the effectiveness of chemotherapy. ${ }^{97}$ Limitations associated with these approaches are mainly inherent in the short timing available for testing, since a major loss of tumor viability, particularly for carcinoma tissues rapidly occurs following surgical excision. It is tempting to speculate that tumor fragments might serve as precious tools to assess the effectiveness of anticancer treatments prior to their administration to patients. A similar approach would likely require the establishment of innovative culture approaches preserving viability and functional potential of the different cell types included in the tumor microenvironment for time periods allowing the elicitation of anticancer immune effects. 
Indeed, advanced immunotherapy protocols utilizing biologicals targeting immunological checkpoints presently provide significant benefit to sizable fractions of treated patients, varying in cancers of different histological origin. However, these treatments are also characterized by a high incidence of severe adverse events. Although the identification of markers predicting responsiveness currently represents an active research area ${ }^{10}$ relatively large numbers of patients undergo highly toxic treatments without clinical benefit. Personalized in vitro models could help to identify responsive patients prior to the initiation of therapy and novel combination approaches.

On the other hand, fragments from clinical specimens cannot be used for high throughput screening and may only be utilized to validate data emerging from less heterogeneous and more standardized models. Therefore, the establishment of more complex and realistic models of the tumor immune system interaction in vitro still represents a challenge (Figure 4).

\section{CONCLUSIONS}

It is all too obvious that in vitro models will never reproduce the enormous complexity of cancer growth in vivo. Nevertheless, they might provide the opportunity to test, in highly controlled conditions, basic science hypotheses and innovative treatments. The major advances of the past two decades have boosted an enormous interest in tumor immunobiology and immunotherapy, leading to unprecedented numbers of preclinical and clinical studies. Assessment of the effectiveness of innovative treatments will require the establishment of innovative in vitro technologies. Remarkably, the potential toxicity of these treatments will also have to be tested. Cytokine release and tumor lysis syndromes, and on target/off tumor reactivity do represent major concerns in this area and also urge the establishment of adequate in vitro models.

On the other hand, the analysis of tumor genomes and of the tumor microenvironment is challenging current tumor classification and staging criteria, usually underlying the selection of patients for standard therapeutic protocols. The emerging quest for personalized treatments might provide an additional incentive for the development of innovative culture technologies.

On the basis of this background it is easy to predict a bright future for the in vitro modeling of tumor immune-system interactions.

\section{AUTHOR INFORMATION}

\section{Corresponding Author}

*E-mail: giulio.spagnoli@usb.ch/gcspagnoli@gmail.com. Tel.: +4161265 2378. Fax: +41612653990.

\section{ORCID}

Manuele G. Muraro: 0000-0002-4590-1916

\section{Author Contributions}

${ }^{\#}$ C.M. and M.G.M. contributed equally.

\section{Funding}

This work was partially funded by the Swiss National Fond for Scientific Research (Grant No. 310030_149745 to GCS).

\section{Notes}

The authors declare the following competing financial interest(s): MGM and GCS are shareholders of a company that produces bioreactors for cell culture.

\section{REFERENCES}

(1) Vesely, M. D.; Kershaw, M. H.; Schreiber, R. D.; Smyth, M. J. Natural innate and adaptive immunity to cancer. Annu. Rev. Immunol. 2011, 29, 235-71.

(2) Mantovani, A.; Allavena, P.; Sica, A.; Balkwill, F. Cancer-related inflammation. Nature 2008, 454 (7203), 436-444.

(3) Schreiber, R. D.; Old, L. J.; Smyth, M. J. Cancer immunoediting: integrating immunity's roles in cancer suppression and promotion. Science 2011, 331 (6024), 1565-1570.

(4) Hanahan, D.; Weinberg, R. A. Hallmarks of cancer: the next generation. Cell 2011, 144 (5), 646-674.

(5) Fridman, W. H.; Pages, F.; Sautes-Fridman, C.; Galon, J. The immune contexture in human tumours: impact on clinical outcome. Nat. Rev. Cancer 2012, 12 (4), 298-306.

(6) Topalian, S. L.; Drake, C. G.; Pardoll, D. M. Targeting the PD-1/ B7-H1(PD-L1) pathway to activate anti-tumor immunity. Curr. Opin. Immunol. 2012, 24 (2), 207-12.

(7) Weiner, G. J. Building better monoclonal antibody-based therapeutics. Nat. Rev. Cancer 2015, 15 (6), 361-70.

(8) Golay, J.; Introna, M. Mechanism of action of therapeutic monoclonal antibodies: promises and pitfalls of in vitro and in vivo assays. Arch. Biochem. Biophys. 2012, 526 (2), 146-53.

(9) Furness, A. J.; Vargas, F. A.; Peggs, K. S.; Quezada, S. A. Impact of tumour microenvironment and $\mathrm{FC}_{\mathcal{C}}$ receptors on the activity of immunomodulatory antibodies. Trends Immunol. 2014, 35 (7), 290-8.

(10) Topalian, S. L.; Taube, J. M.; Anders, R. A.; Pardoll, D. M. Mechanism-driven biomarkers to guide immune checkpoint blockade in cancer therapy. Nat. Rev. Cancer 2016, 16 (5), 275-87.

(11) Golay, J.; Da Roit, F.; Bologna, L.; Ferrara, C.; Leusen, J. H.; Rambaldi, A.; Klein, C.; Introna, M. Glycoengineered CD20 antibody obinutuzumab activates neutrophils and mediates phagocytosis through CD16B more efficiently than rituximab. Blood 2013, 122 (20), 348291.

(12) Mossner, E.; Brunker, P.; Moser, S.; Puntener, U.; Schmidt, C.; Herter, S.; Grau, R.; Gerdes, C.; Nopora, A.; van Puijenbroek, E.; Ferrara, C.; Sondermann, P.; Jager, C.; Strein, P.; Fertig, G.; Friess, T.; Schull, C.; Bauer, S.; Dal Porto, J.; Del Nagro, C.; Dabbagh, K.; Dyer, M. J.; Poppema, S.; Klein, C.; Umana, P. Increasing the efficacy of CD20 antibody therapy through the engineering of a new type II anti-CD20 antibody with enhanced direct and immune effector cell-mediated B-cell cytotoxicity. Blood 2010, 115 (22), 4393-402.

(13) Klein, C.; Schaefer, W.; Regula, J. T. The use of CrossMAb technology for the generation of bi- and multispecific antibodies. MAbs 2016, 8 (6), 1010-20.

(14) Pardoll, D. M. The blockade of immune checkpoints in cancer immunotherapy. Nat. Rev. Cancer 2012, 12 (4), 252-64.

(15) Page, D. B.; Postow, M. A.; Callahan, M. K.; Allison, J. P.; Wolchok, J. D. Immune modulation in cancer with antibodies. Annu. Rev. Med. 2014, 65, 185-202.

(16) McCracken, M. N.; Cha, A. C.; Weissman, I. L. Molecular Pathways: Activating T Cells after Cancer Cell Phagocytosis from Blockade of CD47 "Don't Eat Me" Signals. Clin. Cancer Res. 2015, 21 (16), 3597-601.

(17) Weiskopf, K.; Jahchan, N. S.; Schnorr, P. J.; Cristea, S.; Ring, A. M.; Maute, R. L.; Volkmer, A. K.; Volkmer, J. P.; Liu, J.; Lim, J. S.; Yang, D.; Seitz, G.; Nguyen, T.; Wu, D.; Jude, K.; Guerston, H.; Barkal, A.; Trapani, F.; George, J.; Poirier, J. T.; Gardner, E. E.; Miles, L. A.; de Stanchina, E.; Lofgren, S. M.; Vogel, H.; Winslow, M. M.; Dive, C.; Thomas, R. K.; Rudin, C. M.; van de Rijn, M.; Majeti, R.; Garcia, K. C.; Weissman, I. L.; Sage, J. CD47-blocking immunotherapies stimulate macrophage-mediated destruction of small-cell lung cancer. J. Clin. Invest. 2016, 126 (7), 2610-20.

(18) Fesnak, A. D.; June, C. H.; Levine, B. L. Engineered T cells: the promise and challenges of cancer immunotherapy. Nat. Rev. Cancer 2016, 16 (9), 566-81.

(19) Chambers, C. A.; Kuhns, M. S.; Egen, J. G.; Allison, J. P. CTLA-4mediated inhibition in regulation of $\mathrm{T}$ cell responses: mechanisms and manipulation in tumor immunotherapy. Annu. Rev. Immunol. 2001, 19, 565-94. 
(20) Cerottini, J. C.; Nordin, A. A.; Brunner, K. T. Specific in vitro cytotoxicity of thymus-derived lymphocytes sensitized to alloantigens. Nature 1970, 228 (5278), 1308-9.

(21) Coulie, P. G.; Van den Eynde, B. J.; van der Bruggen, P.; Boon, T. Tumour antigens recognized by $T$ lymphocytes: at the core of cancer immunotherapy. Nat. Rev. Cancer 2014, 14 (2), 135-46.

(22) Van der Bruggen, P.; Traversari, C.; Chomez, P.; Lurquin, C.; DePlaen, E.; Van den Eynde, B.; Knuth, A.; Boon, T. A gene encoding an antigen recognized by cytolytic $\mathrm{T}$ lymphocytes on a human melanoma. Science 1991, 254 (5038), 1643-1647.

(23) Topalian, S. L.; Muul, L. M.; Solomon, D.; Rosenberg, S. A. Expansion of human tumor infiltrating lymphocytes for use in immunotherapy trials. J. Immunol. Methods 1987, 102 (1), 127-41.

(24) Adamina, M.; Rosenthal, R.; Weber, W. P.; Frey, D. M.; Viehl, C. T.; Bolli, M.; Huegli, R. W.; Jacob, A. L.; Heberer, M.; Oertli, D.; Marti, W.; Spagnoli, G. C.; Zajac, P. Intranodal immunization with a vaccinia virus encoding multiple antigenic epitopes and costimulatory molecules in metastatic melanoma. Mol. Ther. 2010, 18 (3), 651-659.

(25) Janetzki, S.; Britten, C. M.; Team, M. C. The role of the reporting framework MIATA within current efforts to advance immune monitoring. J. Immunol Methods 2014, 409, 6-8.

(26) Tseng, D.; Volkmer, J. P.; Willingham, S. B.; Contreras-Trujillo, H.; Fathman, J. W.; Fernhoff, N. B.; Seita, J.; Inlay, M. A.; Weiskopf, K.; Miyanishi, M.; Weissman, I. L. Anti-CD47 antibody-mediated phagocytosis of cancer by macrophages primes an effective antitumor T-cell response. Proc. Natl. Acad. Sci. U. S. A. 2013, 110 (27), 11103-8. (27) Zippelius, A.; Batard, P.; Rubio-Godoy, V.; Bioley, G.; Lienard, D.; Lejeune, F.; Rimoldi, D.; Guillaume, P.; Meidenbauer, N.; Mackensen, A.; Rufer, N.; Lubenow, N.; Speiser, D.; Cerottini, J. C.; Romero, P.; Pittet, M. J. Effector function of human tumor-specific CD8 T cells in melanoma lesions: a state of local functional tolerance. Cancer Res. 2004, 64 (8), 2865-2873.

(28) Korman, A. J.; Peggs, K. S.; Allison, J. P. Checkpoint blockade in cancer immunotherapy. Adv. Immunol. 2006, 90, 297-339.

(29) Pietra, G.; Manzini, C.; Rivara, S.; Vitale, M.; Cantoni, C.; Petretto, A.; Balsamo, M.; Conte, R.; Benelli, R.; Minghelli, S.; Solari, N.; Gualco, M.; Queirolo, P.; Moretta, L.; Mingari, M. C. Melanoma cells inhibit natural killer cell function by modulating the expression of activating receptors and cytolytic activity. Cancer Res. 2012, 72 (6), 1407-15.

(30) Sconocchia, G.; Arriga, R.; Tornillo, L.; Terracciano, L.; Ferrone, S.; Spagnoli, G. C. Melanoma cells inhibit NK cell functions. Cancer Res. 2012, 72 (20), 5428-5429.

(31) Condeelis, J.; Pollard, J. W. Macrophages: obligate partners for tumor cell migration, invasion, and metastasis. Cell 2006, 124 (2), 263266.

(32) Mantovani, A.; Sozzani, S.; Locati, M.; Allavena, P.; Sica, A. Macrophage polarization: tumor-associated macrophages as a paradigm for polarized M2 mononuclear phagocytes. Trends Immunol. 2002, 23 (11), 549-555.

(33) Colombo, M. P.; Piconese, S. Regulatory-T-cell inhibition versus depletion: the right choice in cancer immunotherapy. Nat. Rev. Cancer 2007, 7 (11), 880-7.

(34) Gabrilovich, D. I.; Nagaraj, S. Myeloid-derived suppressor cells as regulators of the immune system. Nat. Rev. Immunol. 2009, 9 (3), 16274.

(35) Palazon, A.; Aragones, J.; Morales-Kastresana, A.; de Landazuri, M. O.; Melero, I. Molecular pathways: hypoxia response in immune cells fighting or promoting cancer. Clin. Cancer Res. 2012, 18 (5), 12071213.

(36) Sitkovsky, M.; Lukashev, D. Regulation of immune cells by localtissue oxygen tension: HIF1 alpha and adenosine receptors. Nat. Rev. Immunol. 2005, 5 (9), 712-21.

(37) Atkuri, K. R.; Herzenberg, L. A.; Herzenberg, L. A. Culturing at atmospheric oxygen levels impacts lymphocyte function. Proc. Natl. Acad. Sci. U. S. A. 2005, 102 (10), 3756-9.

(38) Facciabene, A.; Peng, X.; Hagemann, I. S.; Balint, K.; Barchetti, A.; Wang, L. P.; Gimotty, P. A.; Gilks, C. B.; Lal, P.; Zhang, L.; Coukos, G.
Tumour hypoxia promotes tolerance and angiogenesis via CCL28 and $\mathrm{T}(\mathrm{reg})$ cells. Nature 2011, 475 (7355), 226-30.

(39) Bengsch, B.; Johnson, A. L.; Kurachi, M.; Odorizzi, P. M.; Pauken, K. E.; Attanasio, J.; Stelekati, E.; McLane, L. M.; Paley, M. A.; Delgoffe, G. M.; Wherry, E. J. Bioenergetic Insufficiencies Due to Metabolic Alterations Regulated by the Inhibitory Receptor PD-1 Are an Early Driver of CD8(+) T Cell Exhaustion. Immunity 2016, 45 (2), 358-73.

(40) Patsoukis, N.; Bardhan, K.; Chatterjee, P.; Sari, D.; Liu, B.; Bell, L. N.; Karoly, E. D.; Freeman, G. J.; Petkova, V.; Seth, P.; Li, L.; Boussiotis, V. A. PD-1 alters T-cell metabolic reprogramming by inhibiting glycolysis and promoting lipolysis and fatty acid oxidation. Nat. Commun. 2015, 6, 6692.

(41) Chang, C. H.; Qiu, J.; O'Sullivan, D.; Buck, M. D.; Noguchi, T.; Curtis, J. D.; Chen, Q.; Gindin, M.; Gubin, M. M.; van der Windt, G. J.; Tonc, E.; Schreiber, R. D.; Pearce, E. J.; Pearce, E. L. Metabolic Competition in the Tumor Microenvironment Is a Driver of Cancer Progression. Cell 2015, 162 (6), 1229-1241.

(42) Chang, C. H.; Pearce, E. L. Emerging concepts of T cell metabolism as a target of immunotherapy. Nat. Immunol. 2016, 17 (4), 364-8.

(43) Scharping, N. E.; Menk, A. V.; Moreci, R. S.; Whetstone, R. D.; Dadey, R. E.; Watkins, S. C.; Ferris, R. L.; Delgoffe, G. M. The Tumor Microenvironment Represses T Cell Mitochondrial Biogenesis to Drive Intratumoral T Cell Metabolic Insufficiency and Dysfunction. Immunity 2016, 45 (2), 374-88.

(44) Brand, A.; Singer, K.; Koehl, G. E.; Kolitzus, M.; Schoenhammer, G.; Thiel, A.; Matos, C.; Bruss, C.; Klobuch, S.; Peter, K.; Kastenberger, M.; Bogdan, C.; Schleicher, U.; Mackensen, A.; Ullrich, E.; FichtnerFeigl, S.; Kesselring, R.; Mack, M.; Ritter, U.; Schmid, M.; Blank, C.; Dettmer, K.; Oefner, P. J.; Hoffmann, P.; Walenta, S.; Geissler, E. K.; Pouyssegur, J.; Villunger, A.; Steven, A.; Seliger, B.; Schreml, S.; Haferkamp, S.; Kohl, E.; Karrer, S.; Berneburg, M.; Herr, W.; MuellerKlieser, W.; Renner, K.; Kreutz, M. LDHA-Associated Lactic Acid Production Blunts Tumor Immunosurveillance by $\mathrm{T}$ and NK Cells. Cell Metab. 2016, 24 (5), 657-671.

(45) Huang, S. C.; Smith, A. M.; Everts, B.; Colonna, M.; Pearce, E. L.; Schilling, J. D.; Pearce, E. J. Metabolic Reprogramming Mediated by the mTORC2-IRF4 Signaling Axis Is Essential for Macrophage Alternative Activation. Immunity 2016, 45 (4), 817-830.

(46) O’Neill, L. A.; Pearce, E. J. Immunometabolism governs dendritic cell and macrophage function. J. Exp. Med. 2016, 213 (1), 15-23.

(47) Corzo, C. A.; Condamine, T.; Lu, L.; Cotter, M. J.; Youn, J. I.; Cheng, P.; Cho, H. I.; Celis, E.; Quiceno, D. G.; Padhya, T.; McCaffrey, T. V.; McCaffrey, J. C.; Gabrilovich, D. I. HIF-1alpha regulates function and differentiation of myeloid-derived suppressor cells in the tumor microenvironment. J. Exp. Med. 2010, 207 (11), 2439-53.

(48) Yamada, K. M.; Cukierman, E. Modeling tissue morphogenesis and cancer in 3D. Cell 2007, 130 (4), 601-610.

(49) Ghosh, S.; Spagnoli, G. C.; Martin, I.; Ploegert, S.; Demougin, P.; Heberer, M.; Reschner, A. Three-dimensional culture of melanoma cells profoundly affects gene expression profile: a high density oligonucleotide array study. J. Cell. Physiol. 2005, 204 (2), 522-531.

(50) Kim, J. B. Three-dimensional tissue culture models in cancer biology. Semin. Cancer Biol. 2005, 15 (5), 365-77.

(51) Kunz-Schughart, L. A.; Freyer, J. P.; Hofstaedter, F.; Ebner, R. The use of 3-D cultures for high-throughput screening: the multicellular spheroid model. J. Biomol. Screening 2004, 9 (4), 273-85.

(52) Sutherland, R. M.; Macdonald, H. R.; Howell, R. L. Multicellular spheroids: a new model target for in vitro studies of immunity to solid tumor allografts. J.Natl.Cancer Inst. 1977, 58 (6), 1849-1853.

(53) Kelm, J. M.; Timmins, N. E.; Brown, C. J.; Fussenegger, M.; Nielsen, L. K. Method for generation of homogeneous multicellular tumor spheroids applicable to a wide variety of cell types. Biotechnol. Bioeng. 2003, 83 (2), 173-80.

(54) Hirt, C.; Papadimitropoulos, A.; Muraro, M. G.; Mele, V.; Panopoulos, E.; Cremonesi, E.; Ivanek, R.; Schultz-Thater, E.; Droeser, R. A.; Mengus, C.; Heberer, M.; Oertli, D.; Iezzi, G.; Zajac, P.; Eppenberger-Castori, S.; Tornillo, L.; Terracciano, L.; Martin, I.; Spagnoli, G. C. Bioreactor-engineered cancer tissue-like structures 
mimic phenotypes, gene expression profiles and drug resistance patterns observed "in vivo". Biomaterials 2015, 62, 138-146.

(55) Daster, S.; Amatruda, N.; Calabrese, D.; Ivanek, R.; Turrini, E.; Droeser, R. A.; Zajac, P.; Fimognari, C.; Spagnoli, G. C.; Iezzi, G.; Mele, V.; Muraro, M. G. Induction of hypoxia and necrosis in multicellular tumor spheroids is associated with resistance to chemotherapy treatment. Oncotarget 2016, DOI: 10.18632/oncotarget.13857.

(56) Dontu, G.; Abdallah, W. M.; Foley, J. M.; Jackson, K. W.; Clarke, M. F.; Kawamura, M. J.; Wicha, M. S. In vitro propagation and transcriptional profiling of human mammary stem/progenitor cells. Genes Dev. 2003, 17 (10), 1253-70.

(57) Ricci-Vitiani, L.; Lombardi, D. G.; Pilozzi, E.; Biffoni, M.; Todaro, M.; Peschle, C.; De Maria, R. Identification and expansion of human colon-cancer-initiating cells. Nature 2007, 445 (7123), 111-5.

(58) Infanger, D. W.; Lynch, M. E.; Fischbach, C. Engineered culture models for studies of tumor-microenvironment interactions. Annu. Rev. Biomed. Eng. 2013, 15, 29-53.

(59) Wu, M.; Swartz, M. A. Modeling tumor microenvironments in vitro. J. Biomech. Eng. 2014, 136 (2), 021011.

(60) Boussommier-Calleja, A.; Li, R.; Chen, M. B.; Wong, S. C.; Kamm, R. D. Microfluidics: A new tool for modeling cancer-immune interactions. Trends Cancer 2016, 2 (1), 6-19.

(61) Bai, J.; Adriani, G.; Dang, T. M.; Tu, T. Y.; Penny, H. X.; Wong, S. C.; Kamm, R. D.; Thiery, J. P. Contact-dependent carcinoma aggregate dispersion by M2a macrophages via ICAM- 1 and beta2 integrin interactions. Oncotarget 2015, 6 (28), 25295-307.

(62) Pisano, M.; Triacca, V.; Barbee, K. A.; Swartz, M. A. An in vitro model of the tumor-lymphatic microenvironment with simultaneous transendothelial and luminal flows reveals mechanisms of flow enhanced invasion. Integr Biol. (Camb) 2015, 7 (5), 525-33.

(63) Jeon, J. S.; Bersini, S.; Gilardi, M.; Dubini, G.; Charest, J. L.; Moretti, M.; Kamm, R. D. Human 3D vascularized organotypic microfluidic assays to study breast cancer cell extravasation. Proc. Natl. Acad. Sci. U. S. A. 2015, 112 (1), 214-9.

(64) Sacks, P. G.; Taylor, D. L.; Racz, T.; Vasey, T.; Oke, V.; Schantz, S. P. A multicellular tumor spheroid model of cellular immunity against head and neck cancer. Cancer Immunol. Immunother. 1990, 32 (3), 195200.

(65) Green, S. K.; Karlsson, M. C.; Ravetch, J. V.; Kerbel, R. S. Disruption of cell-cell adhesion enhances antibody-dependent cellular cytotoxicity: implications for antibody-based therapeutics of cancer. Cancer Res. 2002, 62 (23), 6891-6900.

(66) Dangles, V.; Validire, P.; Wertheimer, M.; Richon, S.; Bovin, C.; Zeliszewski, D.; Vallancien, G.; Bellet, D. Impact of human bladder cancer cell architecture on autologous T-lymphocyte activation. Int. J. Cancer 2002, 98 (1), 51-56.

(67) Ghosh, S.; Rosenthal, R.; Zajac, P.; Weber, W. P.; Oertli, D.; Heberer, M.; Martin, I.; Spagnoli, G. C.; Reschner, A. Culture of melanoma cells in 3-dimensional architectures results in impaired immunorecognition by cytotoxic $\mathrm{T}$ lymphocytes specific for Melan-A/ MART-1 tumor-associated antigen. Ann. Surg. 2005, 242 (6), 851-7 discussion..

(68) Dangles-Marie, V.; Richon, S.; El-Behi, M.; Echchakir, H.; Dorothee, G.; Thiery, J.; Validire, P.; Vergnon, I.; Menez, J.; Ladjimi, M.; Chouaib, S.; Bellet, D.; Mami-Chouaib, F. A three-dimensional tumor cell defect in activating autologous CTLs is associated with inefficient antigen presentation correlated with heat shock protein-70 downregulation. Cancer Res. 2003, 63 (13), 3682-3687.

(69) Feder-Mengus, C.; Ghosh, S.; Weber, W. P.; Wyler, S.; Zajac, P.; Terracciano, L.; Oertli, D.; Heberer, M.; Martin, I.; Spagnoli, G. C.; Reschner, A. Multiple mechanisms underlie defective recognition of melanoma cells cultured in three-dimensional architectures by antigenspecific cytotoxic T lymphocytes. Br. J. Cancer 2007, 96 (7), 1072-1082. (70) Widmer, D. S.; Hoek, K. S.; Cheng, P. F.; Eichhoff, O. M.; Biedermann, T.; Raaijmakers, M. I.; Hemmi, S.; Dummer, R.; Levesque, M. P. Hypoxia contributes to melanoma heterogeneity by triggering HIF1alpha-dependent phenotype switching. J. Invest. Dermatol. 2013, 133 (10), 2436-2443.
(71) Gottfried, E.; Kunz-Schughart, L. A.; Ebner, S.; Mueller-Klieser, W.; Hoves, S.; Andreesen, R.; Mackensen, A.; Kreutz, M. Tumorderived lactic acid modulates dendritic cell activation and antigen expression. Blood 2006, 107 (5), 2013-2021.

(72) Christakou, A. E.; Ohlin, M.; Onfelt, B.; Wiklund, M. Ultrasonic three-dimensional on-chip cell culture for dynamic studies of tumor immune surveillance by natural killer cells. Lab Chip 2015, 15 (15), $3222-31$.

(73) Augustine, T. N.; Dix-Peek, T.; Duarte, R.; Candy, G. P. Establishment of a heterotypic $3 \mathrm{D}$ culture system to evaluate the interaction of TREG lymphocytes and NK cells with breast cancer. $J$. Immunol. Methods 2015, 426, 1-13.

(74) Giannattasio, A.; Weil, S.; Kloess, S.; Ansari, N.; Stelzer, E. H.; Cerwenka, A.; Steinle, A.; Koehl, U.; Koch, J. Cytotoxicity and infiltration of human NK cells in in vivo-like tumor spheroids. BMC Cancer 2015, 15, 351.

(75) He, W.; Kuang, Y.; Xing, X.; Simpson, R. J.; Huang, H.; Yang, T.; Chen, J.; Yang, L.; Liu, E.; He, W.; Gu, J. Proteomic comparison of 3D and $2 \mathrm{D}$ glioma models reveals increased HLA-E expression in $3 \mathrm{D}$ models is associated with resistance to NK cell-mediated cytotoxicity. J. Proteome Res. 2014, 13 (5), 2272-81.

(76) Adriani, G.; Pavesi, A.; Tan, A. T.; Bertoletti, A.; Thiery, J. P.; Kamm, R. D. Microfluidic models for adoptive cell-mediated cancer immunotherapies. Drug Discovery Today 2016, 21 (9), 1472-8.

(77) Herter, S.; Morra, L.; Schlenker, R.; Sulcova, J.; Fahrni, L.; Waldhauer, I.; Lehmann, S.; Reislander, T.; Agarkova, I.; Kelm, J. M.; Klein, C.; Umana, P.; Bacac, M. A novel three-dimensional heterotypic spheroid model for the assessment of the activity of cancer immunotherapy agents. Cancer Immunol. Immunother. 2017, 66 (1), $129-140$

(78) Pollard, J. W. Tumour-educated macrophages promote tumour progression and metastasis. Nat. Rev. Cancer 2004, 4 (1), 71-8.

(79) Murray, P. J.; Allen, J. E.; Biswas, S. K.; Fisher, E. A.; Gilroy, D. W.; Goerdt, S.; Gordon, S.; Hamilton, J. A.; Ivashkiv, L. B.; Lawrence, T.; Locati, M.; Mantovani, A.; Martinez, F. O.; Mege, J. L.; Mosser, D. M.; Natoli, G.; Saeij, J. P.; Schultze, J. L.; Shirey, K. A.; Sica, A.; Suttles, J.; Udalova, I.; van Ginderachter, J. A.; Vogel, S. N.; Wynn, T. A. Macrophage activation and polarization: nomenclature and experimental guidelines. Immunity 2014, 41 (1), 14-20.

(80) Konur, A.; Kreutz, M.; Knuchel, R.; Krause, S. W.; Andreesen, R. Three-dimensional co-culture of human monocytes and macrophages with tumor cells: analysis of macrophage differentiation and activation. Int. J. Cancer 1996, 66 (5), 645-652.

(81) Konur, A.; Kreutz, M.; Knuchel, R.; Krause, S. W.; Andreesen, R. Cytokine repertoire during maturation of monocytes to macrophages within spheroids of malignant and non-malignant urothelial cell lines. Int. J. Cancer 1998, 78 (5), 648-653.

(82) Fridlender, Z. G.; Sun, J.; Kim, S.; Kapoor, V.; Cheng, G.; Ling, L.; Worthen, G. S.; Albelda, S. M. Polarization of tumor-associated neutrophil phenotype by TGF-beta: "N1" versus "N2" TAN. Cancer Cell 2009, 16 (3), 183-94.

(83) Linde, N.; Gutschalk, C. M.; Hoffmann, C.; Yilmaz, D.; Mueller, M. M. Integrating macrophages into organotypic co-cultures: a 3D in vitro model to study tumor-associated macrophages. PLoS One 2012, 7 (7), e40058.

(84) Rama-Esendagli, D.; Esendagli, G.; Yilmaz, G.; Guc, D. Spheroid formation and invasion capacity are differentially influenced by cocultures of fibroblast and macrophage cells in breast cancer. Mol. Biol. Rep. 2014, 41 (5), 2885-92.

(85) Espinoza-Sanchez, N. A.; Chimal-Ramirez, G. K.; Mantilla, A.; Fuentes-Panana, E. M. IL-1beta, IL-8, and Matrix Metalloproteinases-1, -2 , and -10 Are Enriched upon Monocyte-Breast Cancer Cell Cocultivation in a Matrigel-Based Three-Dimensional System. Front. Immunol. 2017, 8, 205.

(86) Cho, S. W.; Kim, Y. A.; Sun, H. J.; Kim, Y. A.; Oh, B. C.; Yi, K. H.; Park do, J.; Park, Y. J. CXCL16 signaling mediated macrophage effects on tumor invasion of papillary thyroid carcinoma. Endocr.-Relat. Cancer 2016, 23 (2), 113-24. 
(87) Lu, Y.; Li, S.; Ma, L.; Li, Y.; Zhang, X.; Peng, Q.; Mo, C.; Huang, L.; Qin, X.; Liu, Y. Type conversion of secretomes in a 3D TAM2 and HCC cell co-culture system and functional importance of CXCL2 in HCC. Sci. Rep. 2016, 6, 24558.

(88) Miyake, M.; Hori, S.; Morizawa, Y.; Tatsumi, Y.; Nakai, Y.; Anai, S.; Torimoto, K.; Aoki, K.; Tanaka, N.; Shimada, K.; Konishi, N.; Toritsuka, M.; Kishimoto, T.; Rosser, C. J.; Fujimoto, K. CXCL1Mediated Interaction of Cancer Cells with Tumor-Associated Macrophages and Cancer-Associated Fibroblasts Promotes Tumor Progression in Human Bladder Cancer. Neoplasia 2016, 18 (10), 636-646. (89) Li, R.; Hebert, J. D.; Lee, T. A.; Xing, H.; Boussommier-Calleja, A.; Hynes, R. O.; Lauffenburger, D. A.; Kamm, R. D. MacrophageSecreted TNFalpha and TGFbetal Influence Migration Speed and Persistence of Cancer Cells in 3D Tissue Culture via Independent Pathways. Cancer Res. 2017, 77 (2), 279-290.

(90) Parlato, S.; De Ninno, A.; Molfetta, R.; Toschi, E.; Salerno, D.; Mencattini, A.; Romagnoli, G.; Fragale, A.; Roccazzello, L.; Buoncervello, M.; Canini, I.; Bentivegna, E.; Falchi, M.; Bertani, F. R.; Gerardino, A.; Martinelli, E.; Natale, C.; Paolini, R.; Businaro, L.; Gabriele, L. 3D Microfluidic model for evaluating immunotherapy efficacy by tracking dendritic cell behaviour toward tumor cells. Sci. Rep. 2017, 7 (1), 1093.

(91) Jablonska, J.; Leschner, S.; Westphal, K.; Lienenklaus, S.; Weiss, S. Neutrophils responsive to endogenous IFN-beta regulate tumor angiogenesis and growth in a mouse tumor model. J. Clin. Invest. 2010, 120 (4), 1151-64.

(92) Hynes, R. O. The extracellular matrix: not just pretty fibrils. Science 2009, 326 (5957), 1216-9.

(93) Lu, P.; Weaver, V. M.; Werb, Z. The extracellular matrix: a dynamic niche in cancer progression. J. Cell Biol. 2012, 196 (4), 395406.

(94) Gu, L.; Mooney, D. J. Biomaterials and emerging anticancer therapeutics: engineering the microenvironment. Nat. Rev. Cancer 2016, $16(1), 56-66$.

(95) Crapo, P. M.; Gilbert, T. W.; Badylak, S. F. An overview of tissue and whole organ decellularization processes. Biomaterials 2011, 32 (12), 3233-43.

(96) Genovese, L.; Zawada, L.; Tosoni, A.; Ferri, A.; Zerbi, P.; Allevi, R.; Nebuloni, M.; Alfano, M. Cellular localization, invasion, and turnover are differently influenced by healthy and tumor-derived extracellular matrix. Tissue Eng., Part A 2014, 20 (13-14), 2005-2018.

(97) Majumder, B.; Baraneedharan, U.; Thiyagarajan, S.; Radhakrishnan, P.; Narasimhan, H.; Dhandapani, M.; Brijwani, N.; Pinto, D. D.; Prasath, A.; Shanthappa, B. U.; Thayakumar, A.; Surendran, R.; Babu, G. K.; Shenoy, A. M.; Kuriakose, M. A.; Bergthold, G.; Horowitz, P.; Loda, M.; Beroukhim, R.; Agarwal, S.; Sengupta, S.; Sundaram, M.; Majumder, P. K. Predicting clinical response to anticancer drugs using an ex vivo platform that captures tumour heterogeneity. Nat. Commun. 2015, 6, 6169.

(98) Ulrich, T. A.; Jain, A.; Tanner, K.; MacKay, J. L.; Kumar, S. Probing cellular mechanobiology in three-dimensional culture with collagen-agarose matrices. Biomaterials 2010, 31 (7), 1875-84.

(99) Benton, G.; Kleinman, H. K.; George, J.; Arnaoutova, I. Multiple uses of basement membrane-like matrix (BME/Matrigel) in vitro and in vivo with cancer cells. Int. J. Cancer 2011, 128 (8), 1751-1757.

(100) Carvalho, M. P.; Costa, E. C.; Miguel, S. P.; Correia, I. J. Tumor spheroid assembly on hyaluronic acid-based structures: A review. Carbohydr. Polym. 2016, 150, 139-48.

(101) Liu, X. Q.; Kiefl, R.; Roskopf, C.; Tian, F.; Huber, R. M. Interactions among Lung Cancer Cells, Fibroblasts, and Macrophages in 3D Co-Cultures and the Impact on MMP-1 and VEGF Expression. PLoS One 2016, 11 (5), e0156268.

(102) Ou, Z.; Wang, Y.; Liu, L.; Li, L.; Yeh, S.; Qi, L.; Chang, C. Tumor microenvironment $\mathrm{B}$ cells increase bladder cancer metastasis via modulation of the IL-8/androgen receptor (AR)/MMPs signals. Oncotarget 2015, 6 (28), 26065-78.

(103) Fischer, K. M.; Morgan, K. Y.; Hearon, K.; Sklaviadis, D.; Tochka, Z. L.; Fenton, O. S.; Anderson, D. G.; Langer, R.; Freed, L. E.
Poly(Limonene Thioether) Scaffold for Tissue Engineering. Adv. Healthcare Mater. 2016, 5 (7), 813-21.

(104) Kim, T. E.; Kim, C. G.; Kim, J. S.; Jin, S.; Yoon, S.; Bae, H. R.; Kim, J. H.; Jeong, Y. H.; Kwak, J. Y. Three-dimensional culture and interaction of cancer cells and dendritic cells in an electrospun nanosubmicron hybrid fibrous scaffold. Int. J. Nanomed. 2016, 11, 823-35.

(105) Li, W. A.; Mooney, D. J. Materials based tumor immunotherapy vaccines. Curr. Opin. Immunol. 2013, 25 (2), 238-45.

(106) Kumar, M.; Coburn, J.; Kaplan, D. L.; Mandal, B. B. ImmunoInformed 3D Silk Biomaterials for Tailoring Biological Responses. ACS Appl. Mater. Interfaces 2016, 8 (43), 29310-29322.

(107) Kim, J.; Li, W. A.; Choi, Y.; Lewin, S. A.; Verbeke, C. S.; Dranoff, G.; Mooney, D. J. Injectable, spontaneously assembling, inorganic scaffolds modulate immune cells in vivo and increase vaccine efficacy. Nat. Biotechnol. 2015, 33 (1), 64-72.

(108) Sadtler, K.; Estrellas, K.; Allen, B. W.; Wolf, M. T.; Fan, H.; Tam, A. J.; Patel, C. H.; Luber, B. S.; Wang, H.; Wagner, K. R.; Powell, J. D.; Housseau, F.; Pardoll, D. M.; Elisseeff, J. H. Developing a proregenerative biomaterial scaffold microenvironment requires $\mathrm{T}$ helper 2 cells. Science 2016, 352 (6283), 366-70.

(109) Bissell, M. J.; Radisky, D. Putting tumours in context. Nat. Rev. Cancer 2001, 1 (1), 46-54.

(110) Amicarella, F.; Muraro, M. G.; Hirt, C.; Cremonesi, E.; Padovan, E.; Mele, V.; Governa, V.; Han, J.; Huber, X.; Droeser, R. A.; Zuber, M.; Adamina, M.; Bolli, M.; Rosso, R.; Lugli, A.; Zlobec, I.; Terracciano, L.; Tornillo, L.; Zajac, P.; Eppenberger-Castori, S.; Trapani, F.; Oertli, D.; Iezzi, G. Dual role of tumour-infiltrating $\mathrm{T}$ helper 17 cells in human colorectal cancer. Gut 2017, 66, 692.

(111) Albini, A.; Sporn, M. B. The tumour microenvironment as a target for chemoprevention. Nat. Rev. Cancer 2007, 7 (2), 139-47.

(112) Kalluri, R.; Zeisberg, M. Fibroblasts in cancer. Nat. Rev. Cancer 2006, 6 (5), 392-401.

(113) Klemm, F.; Joyce, J. A. Microenvironmental regulation of therapeutic response in cancer. Trends Cell Biol. 2015, 25 (4), 198-213.

(114) Beura, L. K.; Hamilton, S. E.; Bi, K.; Schenkel, J. M.; Odumade, O. A.; Casey, K. A.; Thompson, E. A.; Fraser, K. A.; Rosato, P. C.; FilaliMouhim, A.; Sekaly, R. P.; Jenkins, M. K.; Vezys, V.; Haining, W. N.; Jameson, S. C.; Masopust, D. Normalizing the environment recapitulates adult human immune traits in laboratory mice. Nature 2016, 532 (7600), 512-6.

(115) Hidalgo, M.; Amant, F.; Biankin, A. V.; Budinska, E.; Byrne, A. T.; Caldas, C.; Clarke, R. B.; de Jong, S.; Jonkers, J.; Maelandsmo, G. M.; Roman-Roman, S.; Seoane, J.; Trusolino, L.; Villanueva, A. Patientderived xenograft models: an emerging platform for translational cancer research. Cancer Discovery 2014, 4 (9), 998-1013.

(116) Hauptmann, S.; Zwadlo-Klarwasser, G.; Jansen, M.; Klosterhalfen, B.; Kirkpatrick, C. J. Macrophages and multicellular tumor spheroids in co-culture: a three-dimensional model to study tumor-host interactions. Evidence for macrophage-mediated tumor cell proliferation and migration. Am. J. Pathol. 1993, 143 (5), 1406-1415.

(117) Wu, M.; Swartz, M. Modeling tumor microenvironments in vitro. J. Biomech. Eng. 2014, 136, 021011.

(118) Sung, K. E.; Beebe, D. J. Microfluidic 3D models of cancer. Adv. Drug Delivery Rev. 2014, 79-80, 68-78. 